\title{
Savoir
}

Bernard Dubos, Xavier Bonneau et Albert Flori

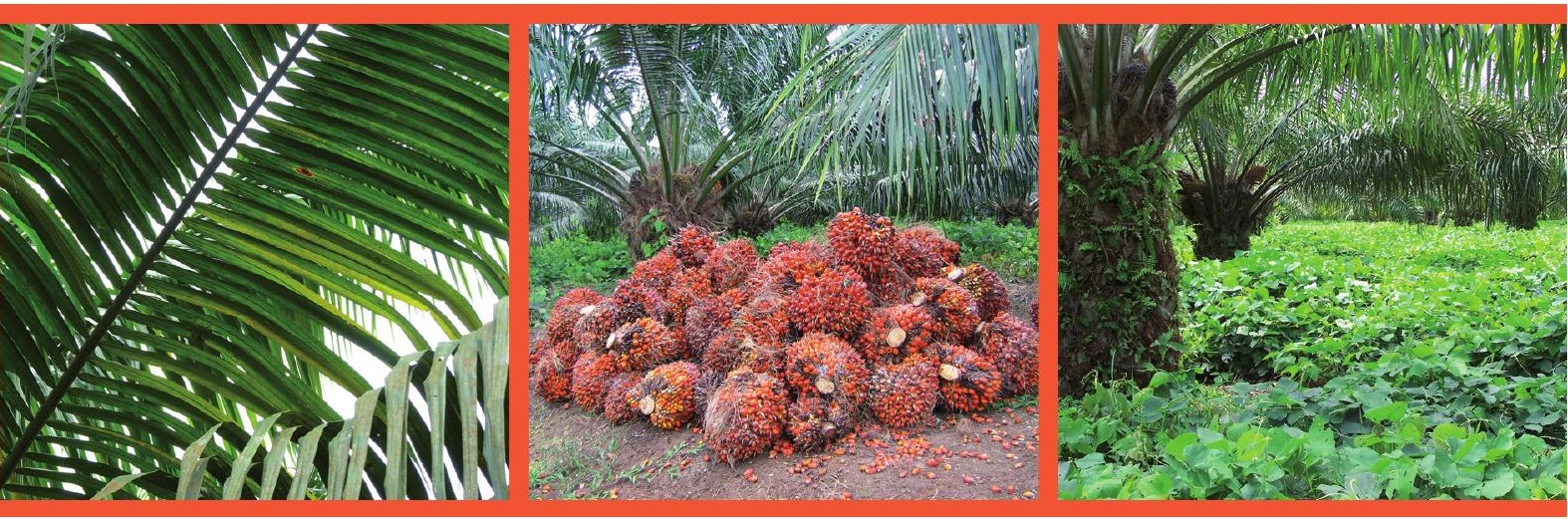

\section{Piloter la fertilisation du palmier à huile}

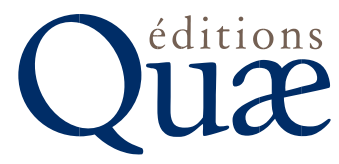





\title{
Piloter la fertilisation du palmier à huile
}

\author{
Bernard Dubos, Xavier Bonneau et Albert Flori
}




\title{
Collection Savoir-faire
}

Quelles alternatives en expérimentation animale?

Pratiques et éthique

F. Marano, P. Hubert, L. Geoffroy, H. Juin

186 pages

Génétique des animaux d'élevage.

Diversité et adaptation dans un monde changeant

E. Verrier, D. Milan, C. Roger-Gaillard

288 pages

Qualité du café.

L'impact du traitement post-récolte

M. Barel

112 pages

\author{
Éditions Quæ \\ RD 10, 78026 Versailles Cedex \\ (C) Éditions Quæ, 2020 \\ ISBN papier : 978-2-7592-3261-1 \\ e-ISBN (pdf) : 978-2-7592-3262-8 \\ x-ISBN (ePub) : 978-2-7592-3263-5 \\ www.quae.com
}

Les versions électroniques de cet ouvrage sont diffusées sous licence CC-by-NCND 2.0.

Le code de la propriété intellectuelle interdit la photocopie à usage collectif sans autorisation des ayants droit. Le non-respect de cette disposition met en danger l'édition, notamment scientifique, et est sanctionné pénalement. Toute reproduction, même partielle, du présent ouvrage est interdite sans autorisation du Centre français d'exploitation du droit de copie (CFC), 20 rue des Grands-Augustins, Paris $6^{\mathrm{e}}$. 


\section{Sommaire}

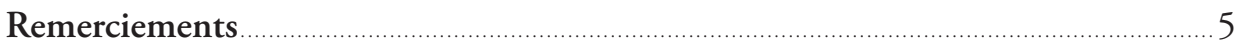

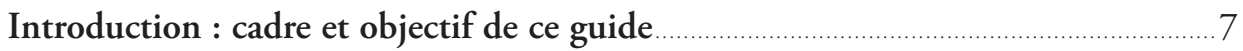

1. Comprendre la nutrition minérale du palmier à huile et le diagnostic des besoins nutritionnels

Pourquoi fertiliser les plantations de palmier à huile? …...............................11

Peut-on se baser sur des symptômes de déficience pour préconiser

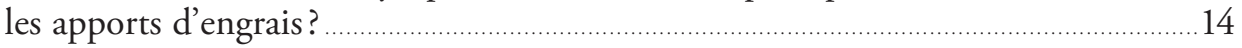

Déficience en azote $(\mathrm{N})$.............................................................................................. 14

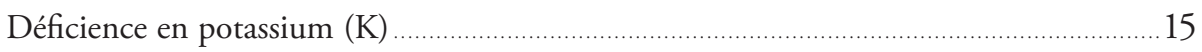

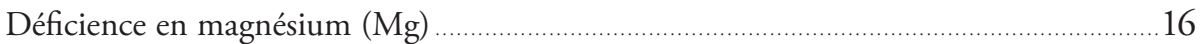

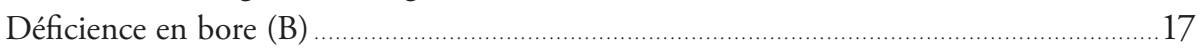

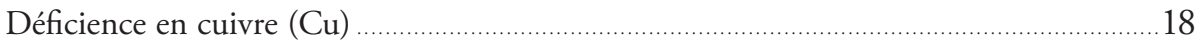

Déficience en manganèse $(\mathrm{Mn})$......................................................................................... 18

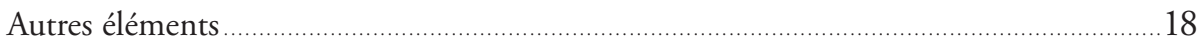

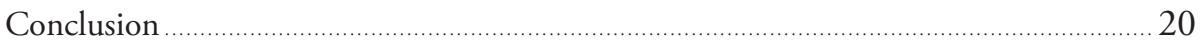

Analyser des échantillons foliaires pour établir un diagnostic ...............................2

Connaître la variabilité des teneurs foliaires en éléments minéraux …......................22

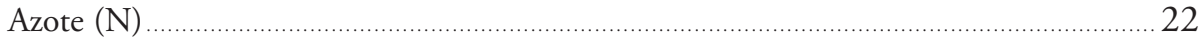

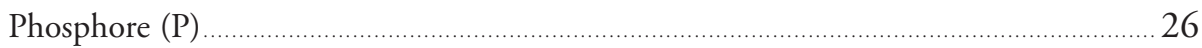

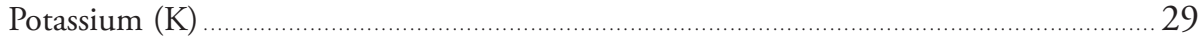

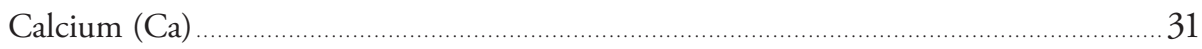

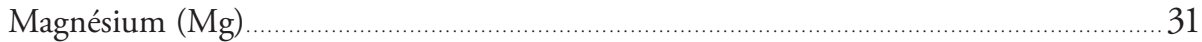

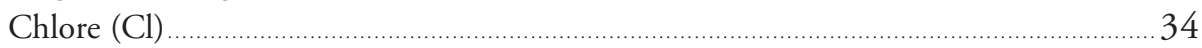

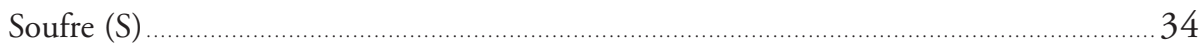

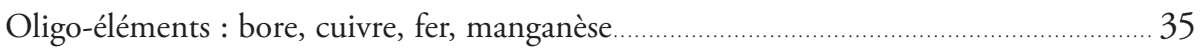

Interpréter les teneurs foliaires en tenant compte des caractéristiques

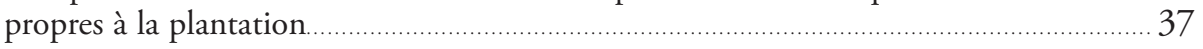

2. Échantillonner la plantation pour le suivi continu de la nutrition minérale ... 39

Découper la plantation en plusieurs unités de fertilisation...................................39

Prévoir le calendrier des prélèvements foliaires ................................................................ 41

Choisir les palmiers pour l'échantillon foliaire de référence

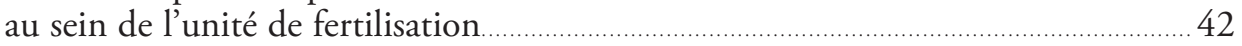

Restreindre l'échantillonnage de référence à une partie homogène de l'unité de fertilisation. 
Illustration des choix d'unités entre faciès non majoritaires......................................... 44

Quand, et sur quels critères, sélectionner les palmiers qui serviront aux prélèvements des échantillons?

Recourir à des échantillons foliaires spécifiques pour contrôler certaines zones de l'unité de fertilisation.

\section{Adapter l'outil d'aide à la décision aux conditions locales :}

\section{prendre en compte les spécificités de chaque site}

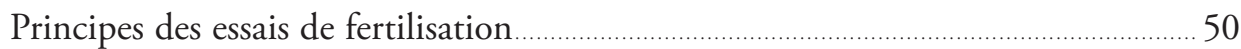

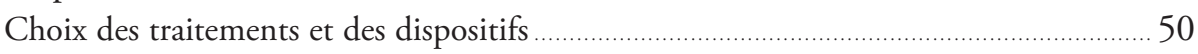

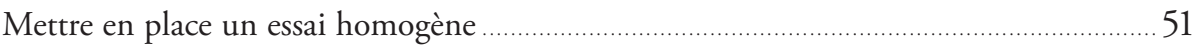

Agréger les données et déterminer les teneurs optimales locales .....................................54

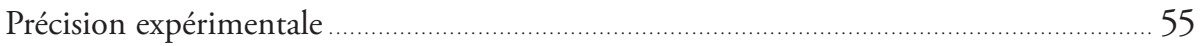

Construire la table de fertilisation à partir des résultats expérimentaux ……............56

Déterminer la plage des teneurs optimales pour un élément ......................................56 56

Construire une table de fertilisation à partir de la plage des teneurs optimales .............. 59

Exploiter les conclusions de l'approche expérimentale .............................................6 60

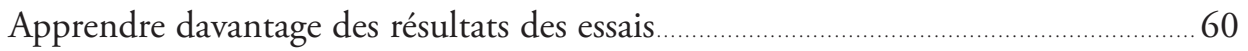

4. Extrapolation de l'application des tables de fertilisation issues des essais ........ 63

Analyser le comportement de la plantation à l'échelle des unités de fertilisation... 63

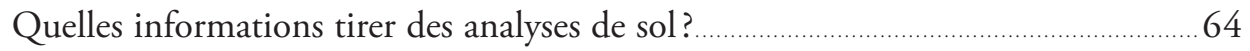

Tenir compte des teneurs du sol en calcium lorsqu'on utilise du $\mathrm{KCl}$.....................65

Comment détecter les perturbations dues à des propriétés des sols ? .........................68

Associer une analyse de référence du sol à chaque échantillon foliaire ……....................68

Construire un système d'information géographique (SIG) ……..............................69

Mettre en place des tests de réactivité ……........................................................69

\section{Adopter des pratiques de fertilisation durables :}

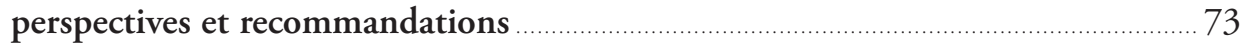

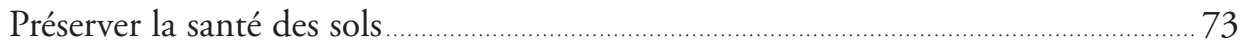

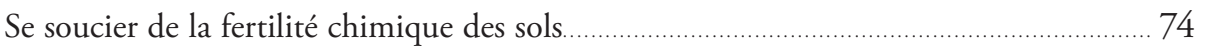

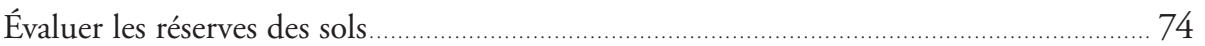

Améliorer l'efficience de la fertilisation ......................................................................... 76

Réduire les pertes en éléments minéraux par différentes pratiques culturales...................76

Améliorer les propriétés physico-chimiques des sols ...................................................78

Élaborer un outil de fertilisation précis et respectueux de l'environnement ............79

Précision des recommandations et des tables de fertilisation ............................................ 79

Précision spatiale de l'échantillonnage foliaire et de l'apport d'engrais ............................ 80

Conclusion : des outils génériques pour optimiser la fertilisation de chaque plantation.

Bibliographie 


\section{Remerciements}

Nous voulons remercier ici Cécile Fovet-Rabot sans qui ce livre n’aurait pas existé, sa lecture critique des premières versions et les suggestions ont permis d'améliorer l'agencement de cet ouvrage et sa lisibilité.

Un grand merci aussi à Bruno Rapidel et Alain Rival pour leur soutien sans réserve et le temps qu'ils ont consacré à la relecture attentive du manuscrit.

Nous remercions également l'UPR systèmes de pérennes et la délégation à l'information scientifique et technique du Cirad pour leur soutien financier à la réalisation de cet ouvrage. 



\section{Introduction : cadre et objectif de ce guide}

Le palmier à huile est devenu la première source mondiale de corps gras d'origine végétale en raison de ses rendements en huile qui sont 4 à 10 fois supérieurs à ceux des autres oléagineux et de ses coûts de production attractifs. Ces atouts expliquent l'augmentation régulière des surfaces cultivées en palmier à huile pour satisfaire la demande mondiale croissante en corps gras, et particulièrement celle des pays émergents et en voie de développement (Asie du Sud-Est, Chine, Inde, Afrique).

\section{Encadré 1. Fertilisation et facteurs de productivité.}

La fertilisation est l'un des facteurs de productivité parmi tous ceux qui déterminent le rendement du palmier à huile (figure 1). Pour un matériel végétal donné, la production de régimes est principalement déterminée par l'efficience de la photosynthèse. Celle-ci peut être limitée par le bilan hydrique (sécheresse du sol réduisant les échanges gazeux) et le rayonnement (ensoleillement insuffisant, en particulier lorsque l'alimentation en eau est correcte). La qualité du feuillage est aussi un facteur important, les défoliations sévères causées par les insectes ayant un impact sur la production de photosynthétats (sucres issus de la photosynthèse).

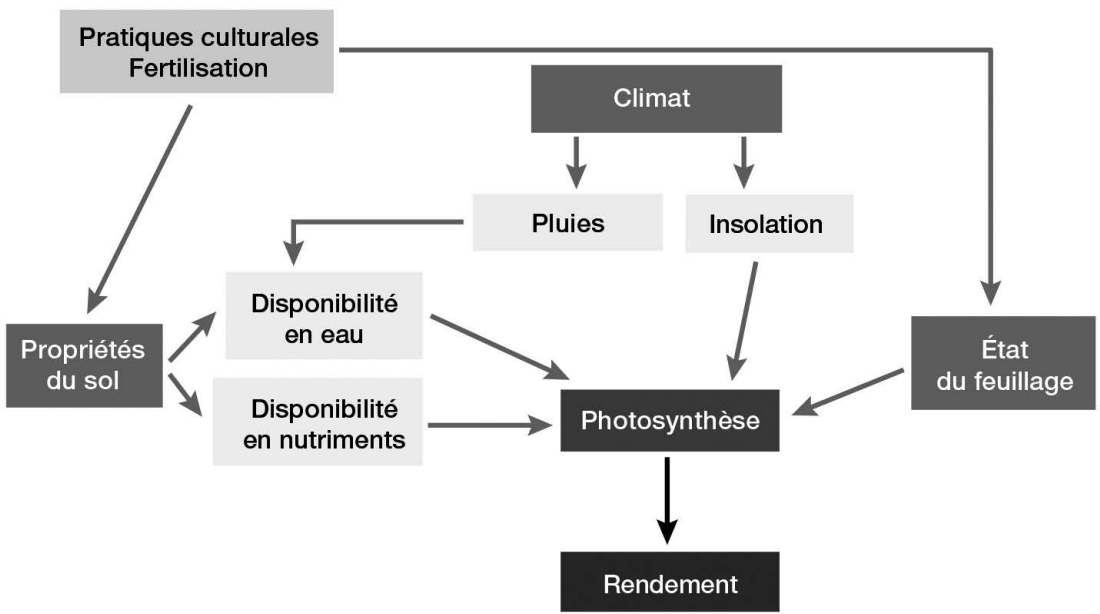

Figure 1. Schéma simplifié de l'élaboration du rendement en régimes du palmier à huile.

Les pratiques agronomiques, incluant la fertilisation, les propriétés du sol, les données climatiques et l'état du feuillage agissent sur la photosynthèse, et en conséquence sur la production des régimes. Ce schéma ne prend pas en compte le temps de réponse d'environ deux ans qui sépare les périodes de stress de leur répercutions sur le rendement. 
L'accroissement en surface des palmeraies génère toutefois des conflits environnementaux et sociaux, chaque fois que cela détruit des forêts tropicales et de la biodiversité. L'amélioration des rendements par voie génétique et par des pratiques agronomiques adaptées permet de répondre à la demande tout en limitant les risques de déforestation. La fertilisation des palmeraies (engrais minéraux ou organiques) a longtemps été considérée comme un moyen majeur d'augmenter la productivité : en effet, les nutriments ne devaient pas être un facteur limitant et des doses d'engrais élevées ont parfois été recommandées (encadré 1). Aujourd'hui, les bonnes pratiques de respect de l'environnement et l'attention aux coûts des intrants agricoles plaident pour une fertilisation raisonnée basée sur un diagnostic précis des besoins de chaque parcelle cultivée.

Un cycle complet de palmier à huile est caractérisé par une croissance continue de la longueur des feuilles jusqu’à 12 ans et du tronc dont la hauteur finale détermine la fin de l'exploitation et la programmation d'un nouveau cycle. Ces particularités doivent être prises en compte dans les décisions de fertilisation au cours des différentes périodes du cycle (figure 2).

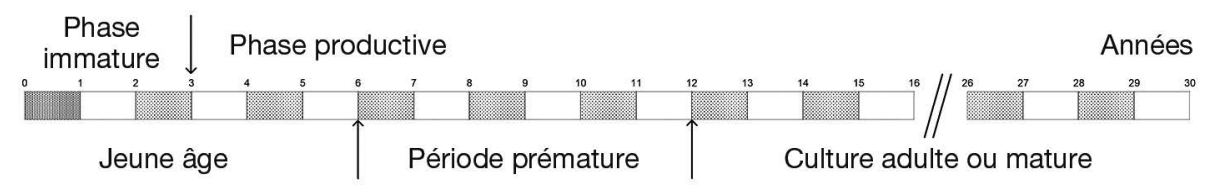

Figure 2. Schéma standard d'un cycle d'exploitation du palmier à huile.

\begin{abstract}
Après la plantation au champ, les 30 à 36 premiers mois constituent la phase immature où les petits régimes ne sont pas récoltés pour des raisons économiques. Les jeunes palmiers sont fertilisés selon un barème propre à chaque plantation. La phase productive commence à partir de 3 ans avec la mise en récolte, période qui coöncide avec le début de la croissance du tronc. Au cours de la phase productive, on analyse des échantillons foliaires pour moduler la fertilisation des parcelles. La longueur des feuilles et de leur biomasse est aussi un bon indicateur. Au jeune âge, jusqu'à 6 ans la croissance est très soutenue jusqu'au moment où les pointes des feuilles horizontales atteignent celles des palmiers voisins. La longueur des feuilles augmente modérément entre 6 et 12 ans, c'est ce que l'on nomme la phase prémature. On considère qu'à partir de 12 ans la biomasse des feuilles est stable : c'est la période adulte (ou mature) qui dure jusqu'à ce qu'il ne soit plus possible de récolter les régimes lorsque la hauteur du tronc atteint 12 mètres, généralement entre 27 et 30 ans. Les rendements en régimes atteignent généralement leur valeur maximale au cours de la phase prémature. Ils restent stables au cours de la période adulte avec parfois une légère baisse après 20 ans.
\end{abstract}

Étayé par une information technique robuste obtenue par de nombreuses années (40 ans) d'expérimentations multi-locales, cet ouvrage est conçu pour guider les agronomes en charge de concevoir des programmes de fertilisation. Il propose de définir dans chaque plantation des recommandations d'engrais qui prennent en compte les spécificités des palmeraies et qui sont fondées sur l'interprétation de résultats d'analyse des tissus végétaux, par le diagnostic foliaire (DF) (encadré 2). 


\section{Encadré 2. Le diagnostic foliaire : un outil très répandu.}

À partir de 1950, les analyses foliaires ont été largement utilisées pour piloter les grandes plantations agro-industrielles.

Les teneurs en éléments minéraux sont exprimées en fonction du poids de matière sèche des folioles (en\% pour $\mathrm{N}, \mathrm{P}, \mathrm{K}, \mathrm{Ca}, \mathrm{Mg}, \mathrm{Cl}$, S, en ppm pour les oligo-éléments). Les résultats des analyses sont comparés à des valeurs de référence, ce qui permet d'ajuster chaque année la fertilisation selon le quadrillage de la plantation en unités de fertilisation (UDF). Le diagnostic foliaire est un outil d'aide à la décision très répandu, mais il est considéré comme empirique. Il reste cependant très largement utilisé en raison de sa facilité de mise en œuvre et de la qualité de l'information qu'il fournit.

Tableau 1. Gammes de teneurs couramment mesurées dans les folioles de la feuille de rang 17.

\begin{tabular}{lcc}
\hline Élément & Symbole & Teneurs \\
\hline Azote & $\mathrm{N}$ & $2,40-3,00 \% \mathrm{~ms}$ \\
\hline Phosphore & $\mathrm{P}$ & $0,15-0,17 \% \mathrm{~ms}$ \\
\hline Potassium & $\mathrm{K}$ & $0,70-1,00 \% \mathrm{~ms}$ \\
\hline Calcium & $\mathrm{Ca}$ & $0,25-0,70 \% \mathrm{~ms}$ \\
\hline Magnésium & $\mathrm{Ca}$ & $0,18-0,22 \% \mathrm{~ms}$ \\
\hline Chlore & $\mathrm{Cl}$ & $0,40-0,70 \% \mathrm{~ms}$ \\
\hline Soufre & $\mathrm{S}$ & $0,18-0,23 \% \mathrm{~ms}$ \\
\hline Bore & $\mathrm{B}$ & $8-15 \mathrm{ppm}$ \\
\hline Cuivre & $\mathrm{Cu}$ & $5-15 \mathrm{ppm}$ \\
\hline Zinc & $\mathrm{Zn}$ & $15-40 \mathrm{ppm}$ \\
\hline Manganèse & $\mathrm{Mn}$ & $100-600 \mathrm{ppm}$ \\
\hline
\end{tabular}

Les teneurs mesurées dépendent des engrais apportés mais aussi d'autres facteurs (climat, sols, matériel végétal).

Ce guide propose de rendre l'outil du diagnostic foliaire plus précis en améliorant chaque étape de son fonctionnement :

- en normalisant tout ce qui peut l'être, c'est-à-dire les modalités d'échantillonnage, la période de prélèvement, le choix du laboratoire;

- en faisant des choix pragmatiques et non systématiques sur le découpage des plantations en unités de fertilisation et sur l'emplacement des palmiers à échantillonner; - en interprétant les diagnostics foliaires selon des teneurs optimales établies en mettant en place des essais de fertilisation dans le même contexte de sol, de climat et de matériel végétal.

Ces protocoles réfléchis et standardisés garantissent la qualité des résultats d'analyse pour le pilotage de surfaces les plus homogènes possible et fournissent une interprétation pertinente des teneurs foliaires pour établir les recommandations d'engrais.

Ce guide explique comment construire des barèmes de fertilisation à partir des résultats des essais de fertilisation et pourquoi on doit les valider par le suivi des réponses des unités de fertilisation de la plantation. 
Il montre également que l'objectif de la fertilisation des palmeraies ne doit pas être perçu comme une simple nécessité d'ajuster un facteur qui pourrait être limitant. En effet, les essais permettent, d'une part, de définir les doses de maintien ou de redressement des teneurs foliaires et, d'autre part, de fixer des seuils au-delà desquels il devient inutile d'appliquer des engrais. Fournir des outils pour une fertilisation précise et respectueuse de l'environnement répond aux attentes de la société en termes de durabilité. Il s'agit de rechercher une optimisation économique et environnementale des pratiques de fertilisation. 


\section{1 \\ Comprendre la nutrition minérale du palmier à huile et le diagnostic des besoins nutritionnels}

Les éléments minéraux nécessaires pour atteindre des rendements élevés sont prélevés de manière différentielle dans l'environnement de la plante et leurs teneurs sont donc différentes selon les organes végétaux.

Lorsque ces éléments indispensables ne sont pas disponibles en quantité suffisante, des déficiences se mettent en place. Les symptômes de déficience concernent généralement de petits groupes de palmiers et leur intensité varie selon les individus. Néanmoins, les observations au champ ne sont pas suffisantes pour prévoir et appliquer une fertilisation corrective à l'échelle de la parcelle ou de l'unité de fertilisation.

Les analyses foliaires sont très utilisées comme moyen de contrôle pour garantir un état nutritionnel correct. Cependant, de nombreux facteurs peuvent agir sur les teneurs foliaires indépendamment de la fertilisation et ils doivent être pris en compte pour l'adoption de normes d'interprétation des teneurs.

\section{Pourquoi fertiliser les plantations de palmier à huile?}

La nutrition minérale est correcte lorsque les éléments nécessaires au bon fonctionnement physiologique du palmier sont absorbés en quantité suffisante pour atteindre le rendement potentiel de chaque site. Dans le cas contraire, une déficience se met en place; elle peut se manifester ou non par des symptômes visibles et elle limite progressivement les rendements. L'intensité des déficiences minérales dépend d'une part des réserves minérales du sol, qui sont liées à sa texture et à ses propriétés conditionnant la capacité de stockage (capacité d'échange de cations CEC, taux de matière organique MO, nature des argiles) et, d'autre part, des apports de fertilisants effectués au cours des années précédentes.

Les essais de fertilisation montrent qu'une déficience s'installe peu à peu et qu'elle se traduit après plusieurs années par une baisse de rendement significative. Ce temps de réponse est variable selon les sites, car il dépend à la fois de la réserve du sol et des prélèvements par la culture dus à la production de biomasse. La fertilisation des palmeraies doit donc intervenir avant que les cultures soient déficientes et l'objectif est de maintenir une composition optimale de la culture qui permette d'atteindre le rendement espéré pour un coût économique et environnemental acceptable. 
L'effet de la fertilisation dépend aussi des autres variables du milieu, qui peuvent devenir des facteurs limitants. Dans des situations très favorables (bon ensoleillement, absence de stress hydrique, faible pression parasitaire), comme dans certaines régions d'Asie du Sud-Est et d'Amérique centrale, la correction des déficiences par la fertilisation aboutit à de fortes hausses de rendement, car les autres facteurs ne sont pas limitants. Au contraire, dans des situations moins optimales, l'effet des engrais est souvent masqué par l'impact d'autres facteurs. Au Bénin par exemple, avec une pluviométrie annuelle moyenne de $1300 \mathrm{~mm}$ (facteur limitant par rapport aux régions citées ci-dessus), les rendements en régimes sont limités à 12 tonnes/ha/an par des déficits hydriques compris entre 600 et $800 \mathrm{~mm} / \mathrm{an}$.

Dans les essais de fertilisation, les variations interannuelles observées pour les rendements ont souvent une amplitude supérieure aux différences observées entre traitements avec ou sans engrais (figure 3). Ces variations d'une année à l'autre se manifestent aussi avec les traitements bien fertilisés. On les attribue aux facteurs de productivité non contrôlés, en particulier aux conditions climatiques.

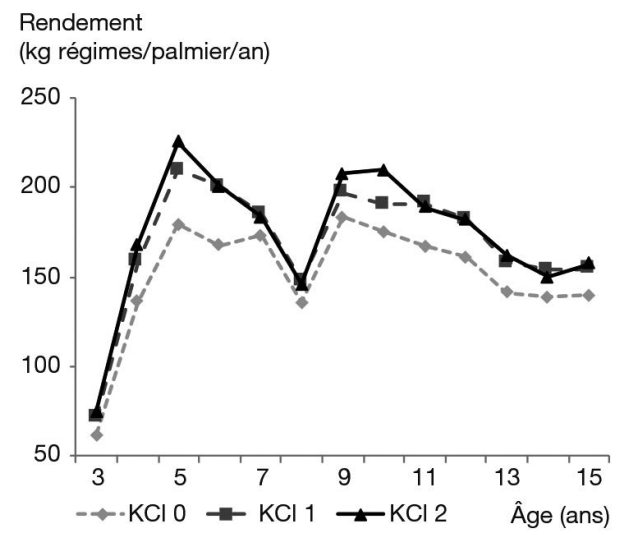

$\mathrm{KCl}$ 0, sans engrais; $\mathrm{KCl} 1,1,5 \mathrm{~kg} /$ palmier/an; $\mathrm{KCl} 2,3 \mathrm{~kg} /$ palmier/an

Figure 3. Exemple de variation interannuelle du rendement en fonction de trois modalités d’apport de chlorure de potassium au Pérou.

Dans cet essai de fertilisation au Pérou étudiant trois modalités d'apports de chlorure de potassium, une baisse du rendement a été enregistrée pour toutes les modalités, à 8 ans. Les résultats ont montré que les modalités $\mathrm{KCl} 1$ et $\mathrm{KCl} \mathrm{2,} \mathrm{permettaient} \mathrm{d'augmenter} \mathrm{les}$ rendements (amélioration significative entre 5 à 7 ans de plantation puis de nouveau de 9 à 15 ans). Mais cet effet a disparu lorsqu'un autre facteur de productivité est devenu limitant : il s'agissait d'un défaut de pollinisation des régimes attribué à une diminution des insectes pollinisateurs.

Que ce soit pour l'espèce Elaeis guineensis ou pour l'hybride Elaeis oleifera $\times$ Elaeis guineensis (dit " $\mathrm{O} \times \mathrm{G} »)$, les éléments nutritifs majeurs à apporter régulièrement pour maintenir des rendements aussi élevés que le permettent les conditions locales, se classent selon l'importance décroissante suivante :

Potassium $(\mathrm{K})>$ Azote $(\mathrm{N})>$ Magnésium $(\mathrm{Mg})>$ Phosphore $(\mathrm{P})$ 
On retrouve cette hiérarchie des éléments dans les quantités présentes dans les parties végétatives (tableau 2) et dans les régimes de palmier à huile (tableau 3). Le chlore $(\mathrm{Cl})$, élément souvent oublié, occupe une position intermédiaire entre le potassium $(\mathrm{K})$ et l'azote $(\mathrm{N})$ dans les tissus végétatifs, mais cet élément n’est pas analysé systématiquement.

À partir de l'âge de 12 ans de la plantation la biomasse de la couronne de feuilles se stabilise et les éléments minéraux contenus dans les feuilles sont restitués au sol lors de l'élagage. Le besoin en éléments minéraux correspond donc à l'accroissement de la biomasse du tronc et à celle des racines mais cette dernière est difficile à évaluer.

Tableau 2. Biomasse sèche et quantités d'éléments minéraux $(\mathrm{kg} / \mathrm{ha})$ présentes dans les parties végétatives aériennes du palmier à huile (Matériel végétal : $E$. guineensis origine Cirad; hybride $\mathrm{O} \times \mathrm{G}$ origine Coari $\times$ La Mé) .

\begin{tabular}{|c|c|c|c|c|c|c|c|}
\hline Matériel & 莣 & 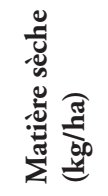 & 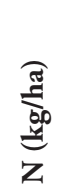 & 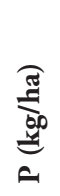 & 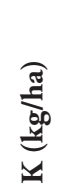 & 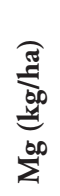 & 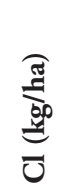 \\
\hline \multirow{3}{*}{$\begin{array}{l}\text { Côte d'Ivoire } \\
\text { E. guineensis } \\
\text { plantation de } 15 \text { ans }\end{array}$} & Tronc & 28228 & 208 & 20 & 386 & 40 & 243 \\
\hline & Couronne $^{(1)}$ & 19022 & 186 & 19 & 198 & 34 & 191 \\
\hline & Total & 47250 & 394 & 39 & 584 & 74 & 434 \\
\hline \multirow{3}{*}{$\begin{array}{l}\text { Équateur } \\
\text { E. guineensis } \\
\text { plantation de } 14 \text { ans }\end{array}$} & Tronc & 31309 & 221 & 14 & 407 & 28 & 274 \\
\hline & Couronne & 14845 & 225 & 17 & 238 & 21 & 218 \\
\hline & Total & 46154 & 446 & 31 & 645 & 49 & 492 \\
\hline \multirow{3}{*}{$\begin{array}{l}\text { Équateur } \\
\text { hybride } \mathrm{O} \times \mathrm{G} \\
\text { plantation de } 13 \text { ans }\end{array}$} & Tronc & 12736 & 178 & 22 & 106 & 16 & 36 \\
\hline & Couronne & 23296 & 250 & 26 & 287 & 52 & 253 \\
\hline & Total & 36032 & 428 & 48 & 393 & 68 & 289 \\
\hline
\end{tabular}

(1) Sur la base de 35 feuilles fonctionnelles par couronne.

Le potassium est le principal élément exporté par les régimes (tableau 3). L’analyse des régimes est une procédure fastidieuse, ce qui explique qu'il y ait peu de résultats disponibles. Or selon leur provenance, les teneurs peuvent varier fortement et cela rend hasardeux l'estimation des quantités d'éléments à remplacer à partir d'une composition standard des régimes. Les données de $\mathrm{Ng}$ et Thamboo sont les plus souvent utilisées bien que ces analyses soient anciennes et caractérisent un matériel végétal de type dura qui n'est plus d'actualité.

À l'âge adulte, les doses d'engrais couramment appliquées pour compenser les exportations varient selon les plantations et les continents :

$-\mathrm{N}, 0,5$ à $1 \mathrm{~kg} / \mathrm{palmier} / \mathrm{an}$;

$-\mathrm{P}, 0,11$ à $0,22 \mathrm{~kg} /$ palmier/an;

- K, 0,5 à $1 \mathrm{~kg} /$ palmier/an;

- Mg, 0,1 à 0,2 kg/palmier/an. 
Tableau 3. Quantités d'éléments minéraux nutritifs ( $\mathrm{kg} /$ tonne de régime) exportées par les régimes, selon différents auteurs.

\begin{tabular}{lccccl}
\hline Référence & $\begin{array}{c}\text { N } \\
(\mathbf{k g} / \mathbf{t})\end{array}$ & $\begin{array}{c}\mathbf{P} \\
(\mathbf{k g} / \mathbf{t})\end{array}$ & $\begin{array}{c}\mathbf{K} \\
(\mathbf{k g} / \mathbf{t})\end{array}$ & $\begin{array}{c}\mathbf{M g} \\
(\mathbf{k g} / \mathbf{t})\end{array}$ & Matériel végétal \\
\hline $\begin{array}{l}\text { Tarmizi A. M. } \\
\text { et Mohd Tayeb D., 2006 } \\
\text { Malaisie }\end{array}$ & 3,10 & 0,37 & 3,92 & 0,68 & E. guineensis tenera \\
\hline $\begin{array}{l}\text { Ng S. K. et Thamboo S., 1967 } \\
\text { Malaisie }\end{array}$ & 2,94 & 0,44 & 3,71 & 0,82 & E. guineensis dura \\
\hline $\begin{array}{l}\text { Teoh K.C. et Chew P.S., 1987 } \\
\text { Malaisie }\end{array}$ & - & - & $4,32-5,12$ & - & $\begin{array}{l}\text { E. guineensis tenera } \\
\text { sur deux types de sol }\end{array}$ \\
\hline $\begin{array}{l}\text { IRHO La Mé, non publié } \\
\text { Côte d'Ivoire }\end{array}$ & 5,70 & 0,81 & 4,26 & 1,19 & E. guineensis tenera \\
\hline $\begin{array}{l}\text { Rincón Numpaque A. H. } \\
\text { et Torres Aguas J. S., 2015 }\end{array}$ & 2,92 & 0,44 & 3,53 & 0,58 & $\begin{array}{l}\text { O } \times \text { G } \\
\text { Colombie }\end{array}$ \\
\hline
\end{tabular}

En Malaisie et en Indonésie - où les rendements peuvent atteindre et dépasser 30 tonnes de régimes $/ \mathrm{ha} / \mathrm{an}$ - il est fréquent d'utiliser 8 à $12 \mathrm{~kg}$ d'engrais (total $\mathrm{N}$, $\mathrm{P}, \mathrm{K}$ et $\mathrm{Mg}$ ) $/$ palmier/an. Les apports sont moins élevés (de 3 à $7 \mathrm{~kg} / \mathrm{palmier} / \mathrm{an}$ ) en Afrique et en Amérique du sud.

Ces écarts justifient qu'on s'interroge sur l'adéquation des doses standards utilisées sur chaque site. Les valeurs maximales ne sont-elles pas trop élevées? Peuvent-elles se révéler dommageables pour l'environnement? Sur quelle information se baser pour moduler la fertilisation dans l'espace et dans le temps afin d'éviter que des déficiences s'installent et limitent les rendements?

\section{Peut-on se baser sur des symptômes de déficience pour préconiser les apports d'engrais?}

Les symptômes de déficience minérale les plus fréquents en plantation sont ceux attribués à $\mathrm{N}, \mathrm{K}, \mathrm{Mg}$ et $\mathrm{B}$. Ils ont été décrits pour la plupart dans plusieurs ouvrages et articles : Fairhurst et Caliman, 2001; Conseils de l'IRHO (Cirad-IRHO, 1969; $1991 ; 1992)$.

Pour certains éléments, les symptômes de déficience peuvent être identifiés sans équivoque mais cela est rarement suffisant pour établir une recommandation de fertilisation précise à l'échelle de la parcelle.

\section{Déficience en azote $(\mathrm{N})$}

La déficience en azote $(\mathrm{N})$ se manifeste par une décoloration diffuse du feuillage qui prend une teinte vert-jaune (photo 1). Sa détection en culture adulte est souvent subjective et dépendante des conditions d'éclairage ce qui limite le diagnostic. La déficience 
est surtout détectable dans la jeune plantation, jusqu’à six ans, quand elle est exacerbée par les facteurs qui limitent les ressources en azote du sol : excès d'eau, absence de légumineuses et densité élevée de graminées. Il faut donc résoudre ces problèmes en même temps que l'on renforce la fertilisation.

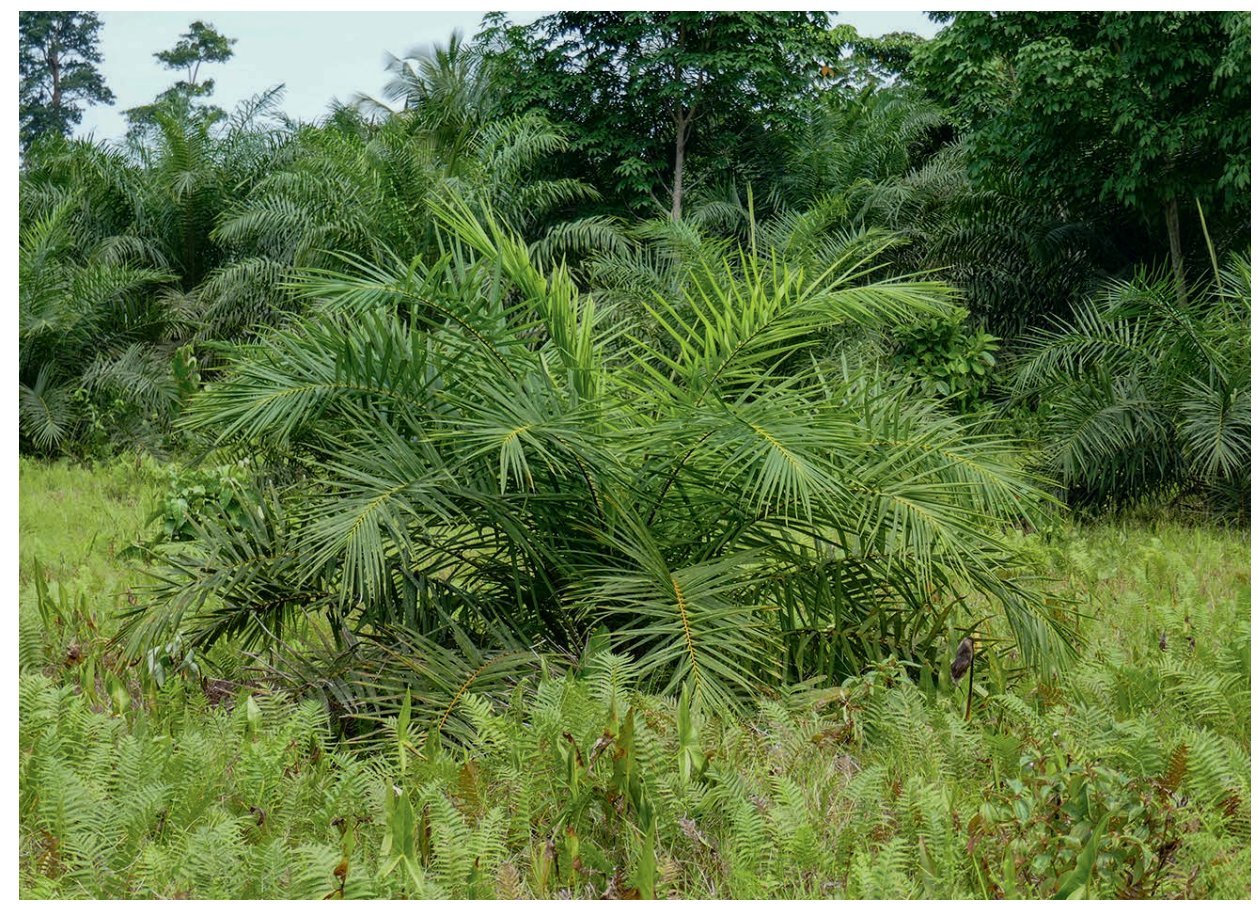

Photo 1. Déficience en azote reconnaissable par la couleur vert pâle du feuillage des palmiers de deux ans en Côte d'Ivoire.

La présence de fougères et un engorgement du sol en saison des pluies diminuent les ressources en azote disponibles pour la culture et ralentissent la croissance des plants.

\section{Déficience en potassium $(K)$}

La déficience en potassium est généralement décrite par deux types de symptômes : - l'apparition de bandes de jaunissement sur les bords des folioles qui s'étendent progressivement à tout le limbe (photo 2);

- l'apparition de petites taches orangées qui contrastent avec la couleur verte des folioles (en anglais : confluent orange spotting).

Ces deux types de symptômes sont observés en essais de fertilisation après plusieurs années de privation de potassium $(\mathrm{K})$, mais leur interprétation en plantation industrielle est délicate. Les bandes de jaunissement n'apparaissent que lorsque les teneurs foliaires ont atteint des niveaux extrêmement faibles $(<0,50 \%)$; cette situation est rare lorsque les plantations sont fertilisées, même si les apports ne sont pas suffisants pour atteindre le meilleur rendement. Les petites taches orangées peuvent être 
confondues avec d'autres dégâts : champignons foliaires de type Cercospora sp., piqûres d'insectes ou d'acariens, anomalies d'origine génétique. Diagnostiquer visuellement une déficience en potassium $(\mathrm{K})$ est un exercice risqué puisqu'on peut conclure que la nutrition est correcte alors qu'elle ne l'est pas et, inversement, conclure à une déficience qui n'existe pas.

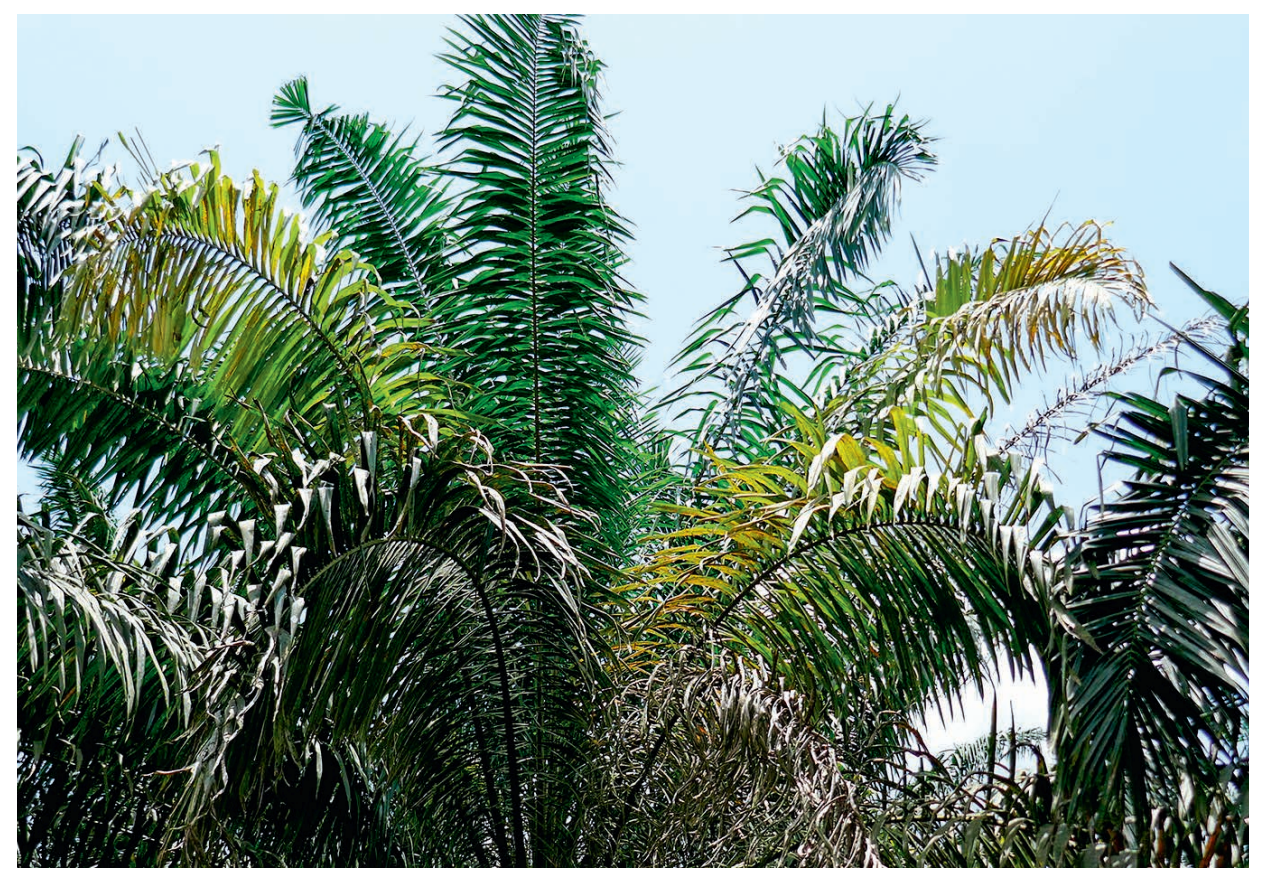

Photo 2. Décoloration des limbes des feuilles de la moyenne couronne dans des plantations villageoises en Côte d'Ivoire.

L'absence d'apports d'engrais pendant de nombreuses années est à l'origine de la déficience en potassium qui, avec ces symptômes, diminue le rendement en régimes.

\section{Déficience en magnésium $(\mathrm{Mg})$}

La déficience en magnésium $(\mathrm{Mg})$ est généralement plus spectaculaire que dommageable. Les plages de décoloration jaune-orangé localisées le plus souvent aux extrémités des feuilles et des folioles apparaissent plus fréquemment en bordure de parcelle. Les symptômes sont renforcés par l'exposition à la lumière : cette particularité en fait aussi un excellent indicateur de déficience, puisque les portions de folioles peu exposées sont plus vertes que le reste du limbe (photo 3). Les symptômes sont présents d'abord sur les feuilles basses avant que l'on observe un effet significatif sur les rendements. Les arbres déficients forment souvent des foyers qui coïncident avec des sols sableux ou caillouteux dont les réserves minérales sont faibles. Pour toutes ces raisons, une application corrective d'engrais ne peut être qu'exceptionnelle et sur de petites surfaces si on ne dispose que de l'observation des symptômes pour appuyer le diagnostic. 

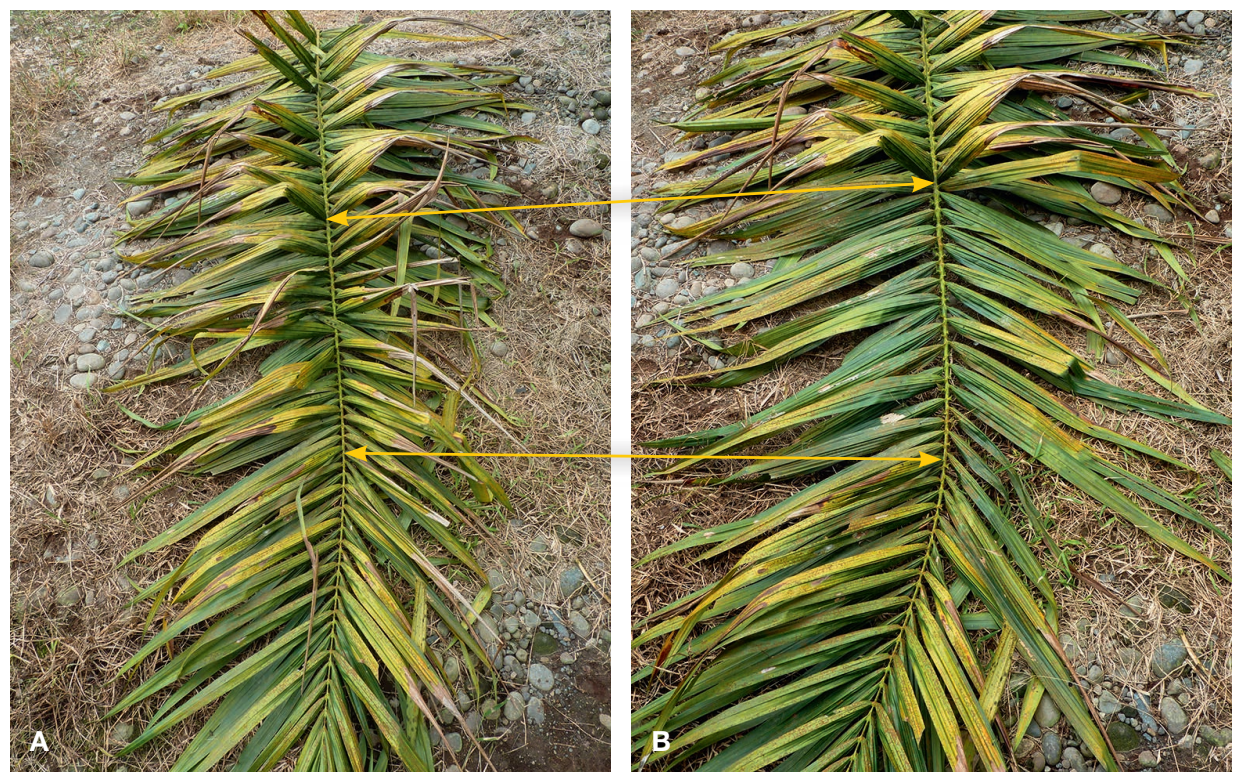

Photo 3. Deux vues (3A et 3B) de la même feuille atteinte d'une déficience en magnésium $(\mathrm{Mg})$ sévère.

Photo 3B : les folioles de la couche supérieure ont été ôtées entre les deux points indiqués par des flèches, laissant apparaître les folioles de la couche inférieure d'un vert beaucoup plus soutenu. Cette différence de teinte des tissus peu ou très exposés à la lumière est caractéristique de la déficience en magnésium.

\section{Déficience en bore $(B)$}

La déficience en bore (B) est difficile à détecter. Les agronomes expérimentés attribuent plusieurs symptômes à cette déficience qui perturbe les apex végétatifs des feuilles avec, par ordre d'intensité croissante, un gaufrage des folioles terminales, des malformations des nervures (baïonnettes) (photo 4) et le raccourcissement des rachis des feuilles jusqu'à une forme de moignon dans les cas graves. On y associe l'apparition plus fréquente de fines bandes de couleur jaune pâle parallèles à la nervure principale des folioles. L'ensemble de ces symptômes indique parfois un vrai problème nutritionnel, en particulier à l'âge sensible, entre 2 et 5 ans. Le critère le plus décisif est l'apparition de nouvelles feuilles jeunes plus courtes que les précédentes, donnant un port tabulaire (Flat Top) aux jeunes plants. À l'inverse, les malformations des folioles terminales ou la présence de quelques bandes décolorées ne constituent pas à elles seules un critère fiable pour établir un besoin en engrais, surtout en culture adulte. La malformation des folioles peut avoir une origine mécanique au moment du déploiement de la feuille, c'est ce que montrent des observations au champ et en pépinière sur du matériel hybride $\mathrm{O} \times \mathrm{G}$ : en effet, ces malformations apparaissent souvent d'un seul côté de la feuille (toujours le même), ce qui suggère qu'ils sont liés à la phyllotaxie du palmier. D'autres causes peuvent être à l'origine des déformations en particulier les dégâts d'insectes. 


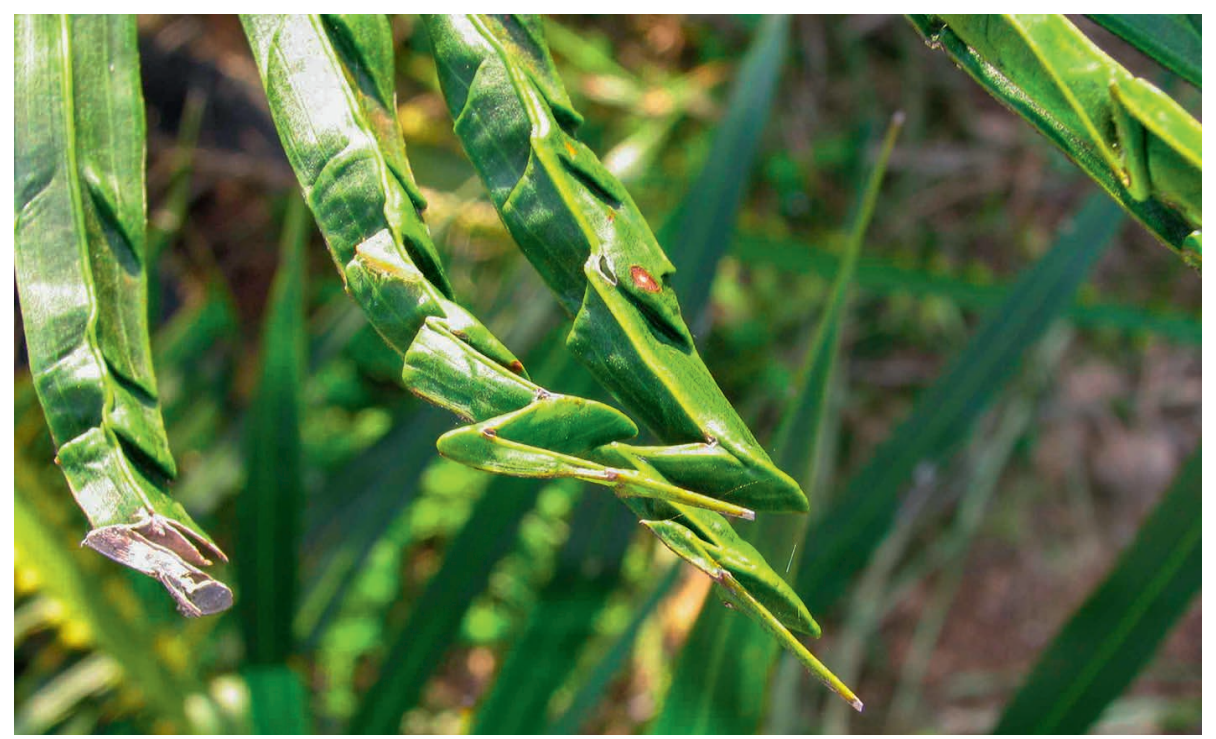

Photo 4. Déformation en baïonnette des extrémités des folioles terminales en raison d'une déficience en bore.

\section{Déficience en cuivre $(\mathrm{Cu})$}

La déficience en cuivre $(\mathrm{Cu})$ a été signalée sur des sols très sableux et dans les palmeraies sur tourbes. Jusqu'à trois ans, elle peut occasionner des pertes de plants si aucun apport de cuivre n'est effectué. Au-delà de cet âge, le risque de déficience en cuivre disparaît. Les premiers symptômes de déficience en cuivre résultent d'une plasmolyse du rachis des jeunes feuilles : le palmier prend une silhouette caractéristique arquée et affaissée (photo 5). Les extrémités des folioles se décolorent par la suite, donnant un dégradé brun-jaune-vert typique. En cas de déficience très sévère, les nouvelles feuilles sont de plus en plus rabougries et cette déficience entraîne parfois la mort du palmier.

\section{Déficience en manganèse $(\mathrm{Mn})$}

La déficience en manganèse (Mn) est rare. Elle apparaît exceptionnellement dans les situations où le calcium, surabondant dans le sol, bloque l'absorption de manganèse. La déficience se manifeste alors par une détérioration des tissus chlorophylliens et un rabougrissement des feuilles; ces symptômes sont faciles à identifier (photo 6). Les individus présentant ces symptômes doivent être fertilisés avec du sulfate de manganèse pour retrouver une croissance et une production normales.

\section{Autres éléments}

Des éléments nutritifs peuvent être déficients et limiter les rendements alors que la déficience est difficilement détectable, comme dans le cas du phosphore (P), voire 


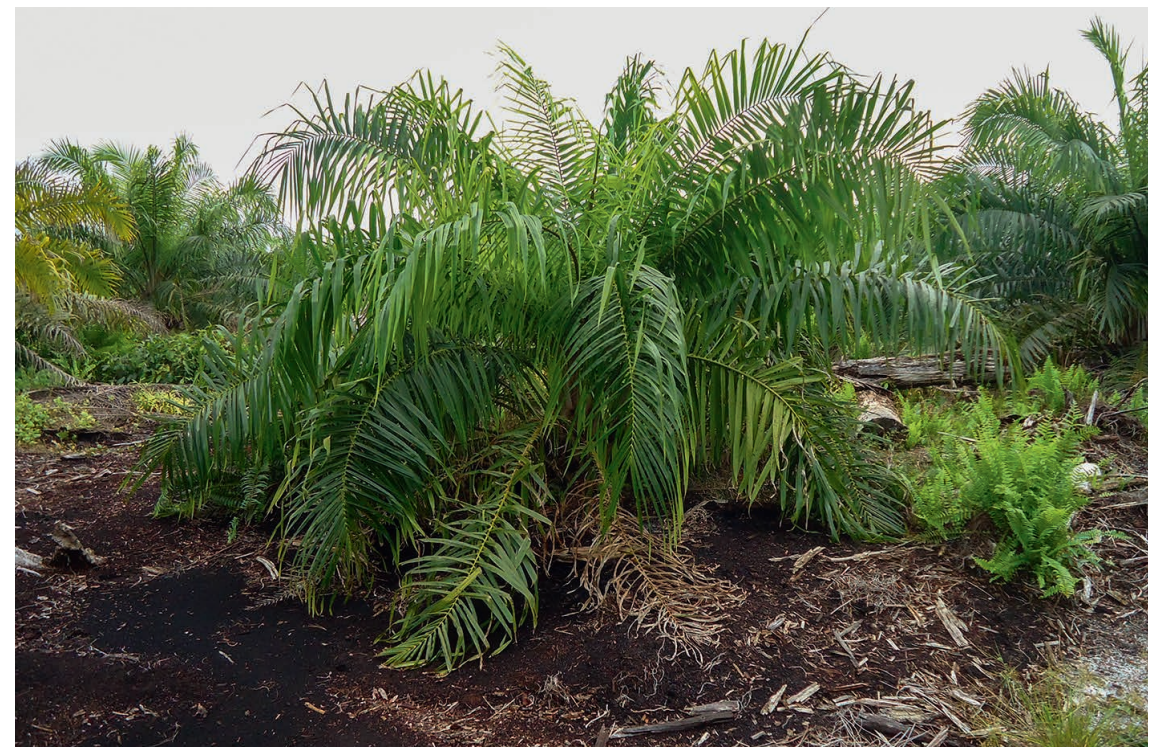

Photo 5. Déficience en cuivre sur tourbe en Indonésie : silhouette arquée et affaissée du palmier.

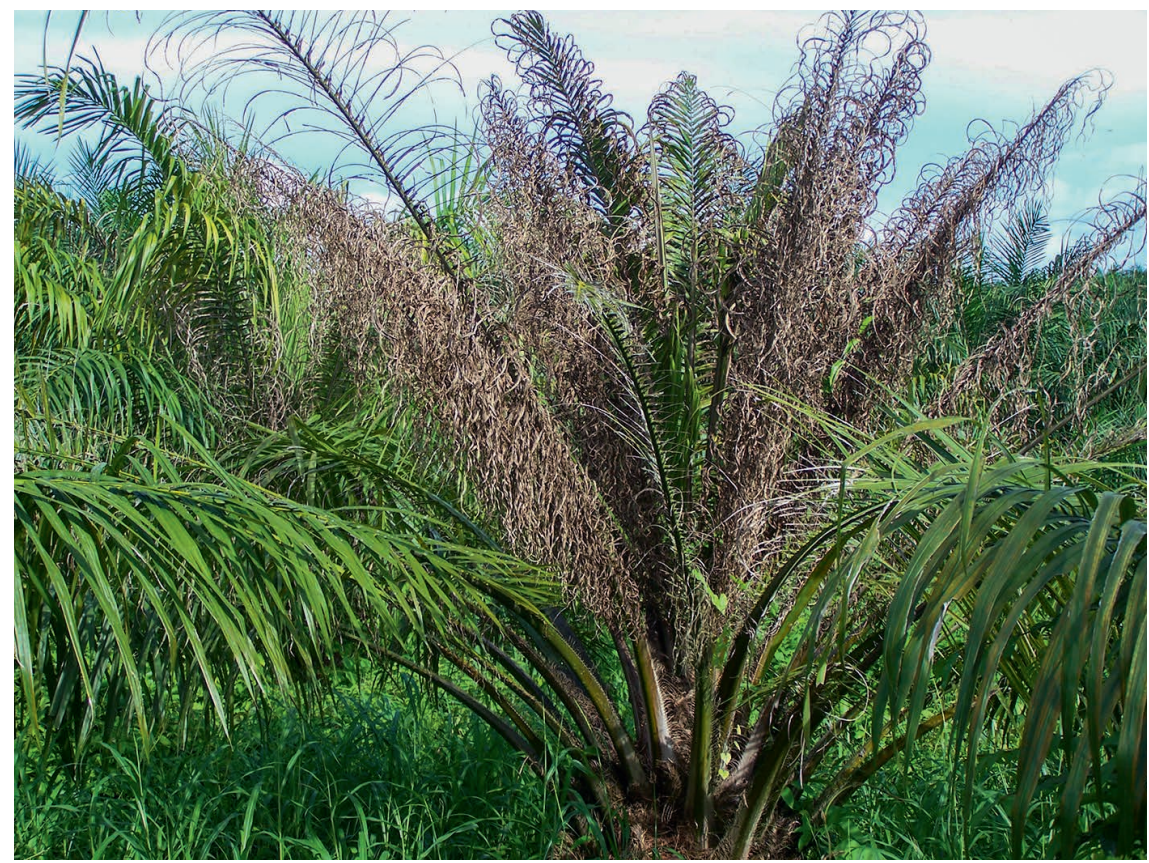

Photo 6. Déficience en manganèse d'un jeune palmier planté sur un relief karstique au Guatemala.

Le dessèchement des nouvelles feuilles peut conduire à la mort du jeune plant si aucun apport de manganèse sous une forme soluble n'est pratiqué. Lorsque cette déficience apparaît, il est aisé de circonscrire les secteurs concernés qui nécessitent un traitement. 
sans symptômes visibles à l'œil nu, comme dans le cas du chlore $(\mathrm{Cl})$. Des déficiences en $\mathrm{P}$ et en $\mathrm{Cl}$ peuvent cependant diminuer sévèrement les rendements.

\section{Conclusion}

L'observation de symptômes de déficience ne renseigne pas précisément sur le besoin d'ajuster la fertilisation.

En phase immature et dans les trois années qui suivent (soit jusqu'à $\mathbf{6}$ ans), la présence de palmiers souffrant d'une déficience permet généralement d'identifier l'élément en cause et d'apprécier l'étendue des surfaces concernées. On n'accorde toutefois pas la même importance au diagnostic s'il s'agit de quelques palmiers ou de plusieurs centaines d'individus affectés. Parce qu'il faut réagir rapidement lorsque les palmiers sont jeunes (réserves minérales moins élevées, racines peu développées) on peut appliquer une fertilisation corrective sur tout ou partie des parcelles selon les éléments et selon l'étendue de la déficience.

En culture de plus de $\mathbf{6}$ ans, on ne peut pas se baser sur la présence de symptômes pour décider d'une recommandation d'engrais, d'une part parce que la nutrition peut être déficiente ( $\mathrm{N}$ et $\mathrm{K}$ en particulier) sans que l'on détecte d'anomalie et, d'autre part, parce qu'il n'y a pas toujours de relation directe entre apparition des symptômes et chute des rendements, comme on a pu l'observer à propos du magnésium.

Ce sont les analyses foliaires qui permettent de confirmer l'existence d'une déficience au champ et elles permettent aussi d'argumenter la prise de décision en fertilisation, même lorsqu'aucun symptôme n'est visible.

\section{Analyser des échantillons foliaires pour établir un diagnostic}

L'outil de diagnostic par analyses foliaires, appelé aussi diagnostic foliaire, renseigne sur la composition du tissu chlorophyllien des folioles ( $d$ 'autres tissus sont parfois utilisés). Il permet de porter un diagnostic en comparant les résultats des teneurs obtenus à des valeurs de référence.

La collecte des échantillons de folioles a été normalisée et les pratiques sont bien partagées par les agronomes et les scientifiques :

- la feuille à prélever est celle située au rang 17 le jour de la prise d'échantillon. Le rang 9 était utilisé autrefois pour établir un premier diagnostic foliaire à 3 ans. Les techniques culturales ayant évolué et permettant dans la majorité des cas de disposer de feuilles de rang 17 saines dès 30 mois, il recommandé de n'utiliser que ce rang; - la position des folioles prélevées est à proximité du milieu du rachis. Ce point est facile à déterminer visuellement, même depuis le sol, et la composition des folioles semble la plus stable dans la partie centrale du limbe. Certains auteurs recommandent aussi le "point B » qui correspond à un changement de forme de la section du rachis (figure 4), mais il est situé sur la face supérieure de la feuille et donc difficilement identifiable pour les grands palmiers. 


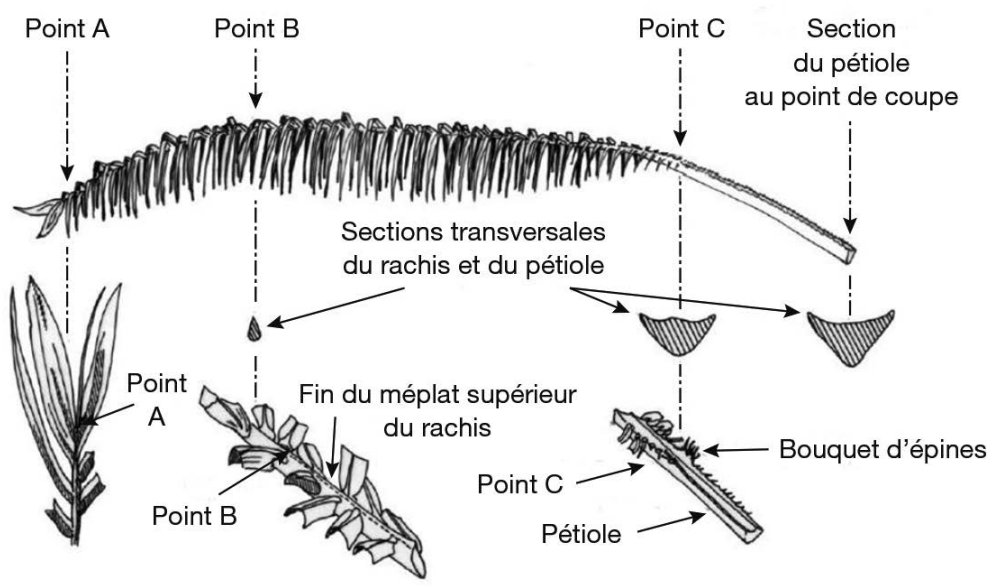

Figure 4. Structure de la feuille du palmier à huile.

Les folioles les plus longues constituant l'échantillon foliaire sont prélevées à mi-distance entre le point $\mathrm{A}$ et le point $\mathrm{C}$. Un fragment de limbe d'une quinzaine de centimètre est coupé dans la partie centrale de quelques folioles et l'échantillon est séché à l'étuve à $70{ }^{\circ} \mathrm{C}$ avant d'être transmis au laboratoire pour analyse.

Le prélèvement de folioles se fait de chaque côté du rachis. Pour les hybrides $\mathrm{O} \times \mathrm{G}$ deux folioles suffisent. Pour le matériel guineensis, deux classes de folioles (plans inférieurs et supérieurs) doivent être représentées, c'est-à-dire qu’on prélèvera au moins 4 folioles par palmier. Ces précautions sont importantes car la teneur en magnésium des folioles varie selon leur position et leur exposition à la lumière (Webb et al., 2009).

On utilise 25 à 35 palmiers pour constituer l'échantillon composite destiné à être analysé, ils sont identifiés au champ et seront prélevés tous les ans. L'effectif de l'échantillon varie principalement en raison des risques sanitaires qui affectent des individus qui disparaissent, ce qui appauvrit progressivement la population initiale.

La localisation des palmiers de l'échantillon dans la parcelle cultivée est primordiale : ce point est détaillé dans la section "Choisir les palmiers pour l'échantillon foliaire au sein de l'unité de fertilisation" page 42.

Lorsque les arbres vieillissent, la feuille de rang 17 est de plus en plus haute et difficile à atteindre (en particulier au point B) avec une faucille. De ce fait, il arrive que l'on coupe la feuille entière pour prélever les folioles. Dans ce cas, l'échantillonnage n'est plus représentatif d'une plantation normalement conduite puisque chaque prélèvement revient à ôter 4 à $5 \%$ des feuilles émises annuellement et à réduire d'autant la surface foliaire. En toute rigueur, ce traitement particulier peut fausser le diagnostic qui est déduit de l'analyse de l'échantillon. Exceptionnellement, ce prélèvement effectué en coupant la feuille entière est acceptable seulement au cours des dernières années avant la replantation.

Pour plus de détails sur le prélèvement des échantillons et leur préparation avant analyse, on peut se référer aux Conseils de l'IRHO (Cirad-IRHO, 1977). 


\section{Connaître la variabilité des teneurs foliaires en éléments minéraux}

\section{Azote (N)}

La teneur en $\mathrm{N}$ des folioles varie entre 2 et $3 \%$ du poids en matière sèche (MS); il est courant de lire qu'une teneur «normale» en $\mathrm{N}$ est de $2,5 \%$ pour Elaeis guineensis. Cette valeur n'a toutefois pas de sens sans référence à l'âge des palmiers, puisque, pour un même rang de feuille, les teneurs diminuent rapidement au cours des premières années à partir de 3 ans (figure 5). Cette diminution, qui est un phénomène commun dans les couverts en croissance, est attribuée à une loi de dilution due à l'accroissement de la biomasse. Chez le palmier, l'influence de l'âge se réduit à partir de 12 ans lorsque les feuilles atteignent une longueur et une biomasse stables, et elle est négligeable à partir de 20 ans.

\section{Les modèles}

Différents modèles ont été mis au point à partir de données d'observation, afin de décrire les variations de la teneur en $\mathrm{N}$ en fonction de l'âge. Ces modèles produisent des valeurs de référence pour aider à gérer la fertilisation (encadré 3). Lorsque les teneurs mesurées atteignent ou dépassent les valeurs du modèle, il n’y a généralement pas d'amélioration du rendement à attendre. Lorsque les teneurs sont inférieures au modèle, on détermine selon le site jusqu’à quelle valeur le déficit est acceptable.

\section{Effet du rang des feuilles sur les teneurs}

Le rang de la feuille prélevée a aussi une influence sur les teneurs en $\mathrm{N}$ (figure 7). Les erreurs de prélèvement pouvant être fréquentes, il est essentiel que les prélèvements soient faits par des personnes maîtrisant parfaitement cette opération.

\section{Encadré 3. Les modèles utilisables pour décrire la variation des teneurs en azote en fonction de l'âge.}

\section{Modèle indonésien : $\mathrm{N}$ Ind}

Tampubolon et al. (1989) ont proposé un modèle dit indonésien, «N Ind », à partir de résultats d'expériences dans le nord de Sumatra (voir également l'exemple en Colombie, figure 5). L'équation est celle d'une parabole :

$N$ Ind $=3,192-0,059 \times n+0,001 \times n^{2}$

$\mathrm{N}$ Ind est la teneur $\mathrm{N}$ (\% MS) calculée par le modèle indonésien, et $\mathrm{n}$ l'âge en années.

\section{Modèle Amérique latine : N AL}

En Amérique latine, le modèle dit «N AL» donne aussi de bons résultats :

$\mathrm{NAL}=2,33+0,7054 \exp (-0,0975 \times \mathrm{n})$

$\mathrm{N}$ AL est la teneur $\mathrm{N}$ (\% MS) calculée par le modèle Amérique latine, et $\mathrm{n}$ l'âge en année. Le modèle $N$ AL est également utilisable pour les hybrides $0 \times G$ (figure 6 ). Dans ce cas, le paramètre constant est ajusté, passant de $2,33 \%$ à $2 \% M S$, ce qui donne l'équation suivante : $\mathrm{N} \mathrm{AL} \mathrm{O} \times \mathrm{G}=2,0+0,7054 \exp (-0,0975 \times \mathrm{n})$

$N$ AL $O \times G$ est la teneur en $N(\% M S)$ calculée par le modèle Amérique latine pour les hybrides $O \times G$, et $n$ l'âge en année. 


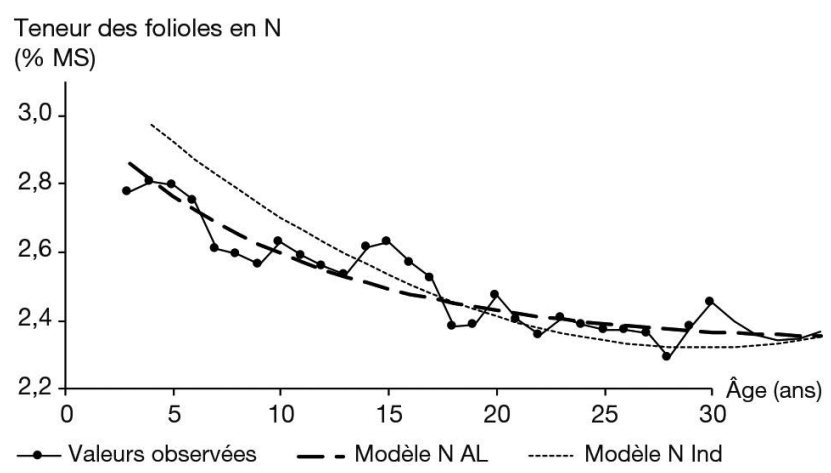

Figure 5. Variations de teneurs en azote des folioles de E. Guineensis pour un cycle complet de culture dans une plantation en Colombie et comparaison aux modèles $\mathrm{N}$ Ind et $\mathrm{N}$ Al.

Les valeurs moyennes ont été obtenues à partir de 4 à 10 valeurs observées pour différentes années de plantation à un âge donné pour lisser l'effet de la fertilisation. Les teneurs décroissent rapidement jusqu'à 12 ans puis modérément au-delà. Cette évolution en fonction de l’âge peut être représentée par les deux modèles mentionnés $\mathrm{N}$ Ind et $\mathrm{N}$ Al. Les écarts par rapport aux teneurs prédites sont dus à d'autres facteurs que l'âge en particulier les conditions climatiques et les cycles de production de biomasse.

L'interprétation des résultats des essais de fertilisation doit prendre en compte un modèle pour faire ressortir la part des variations qui est due au vieillissement des palmiers et celle qui est due à la fertilisation.

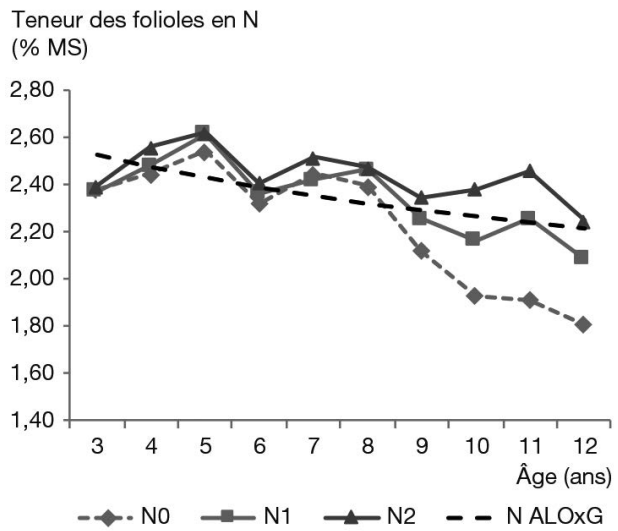

N0 sans engrais; N1, 1,5 kg d'urée / palmier / an; N2, 3 kg d'urée /palmier/an

Figure 6. Résultats d'un essai à trois modalités d'apport d'azote et comparaison au modèle $\mathrm{N} \mathrm{AL} \mathrm{O} \times \mathrm{G}$.

Dans cet essai avec des croisements hybrides $0 \times G$, les teneurs ont baissé jusqu'à 8 ans pour les trois modalités de fertilisation tout en restant supérieures aux valeurs du modèle $\mathrm{NAL} O \times \mathrm{G}$. Ce n'est qu'à partir de 9 ans, que la nutrition azotée du témoin NO s'en est écartée. L'essai a montré qu'à partir de cet âge les rendements moyens observés pour NO sont devenus statistiquement inférieurs à ceux des traitements $\mathrm{N} 1$ et N2. On a donc pu confirmer que l'équation du modèle permettait de prédire une teneur en $\mathrm{N}$ traduisant un état nutritionnel correct à chaque âge. 


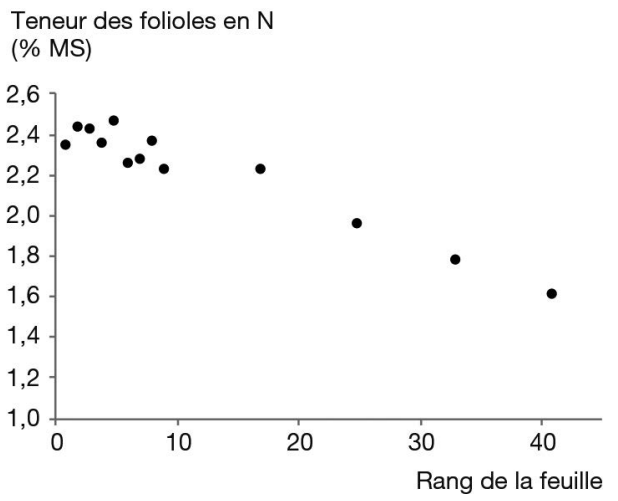

Figure 7. Teneurs en $\mathrm{N}$ en fonction du rang de la feuille pour le croisement LM2T $\times$ DA10D sur la station de La Mé en Côte d'Ivoire.

| On observe une baisse moyenne de 0,02\% MS par rang de feuille.

Pour comparer les teneurs entre deux années successives, il faut respecter rigoureusement le rang des feuilles prélevées, en particulier utiliser le rang 17 dès le premier prélèvement à l'âge de 3 ans.

\section{Effet de l'ensoleillement sur les teneurs}

Bien qu'aucune étude poussée n'ait été faite, il semble que l'ensoleillement annuel influe aussi sur les teneurs en azote. Ce phénomène a été signalé en Équateur où l'ensoleillement très faible observé dans la région de Quinindé (environ $900 \mathrm{~h}$ insolation/an) expliquerait des teneurs systématiquement plus faibles que dans d'autres localités, au même âge, avec une fertilisation équivalente et pour le même matériel végétal, d'origine E. guineensis (figure 8).

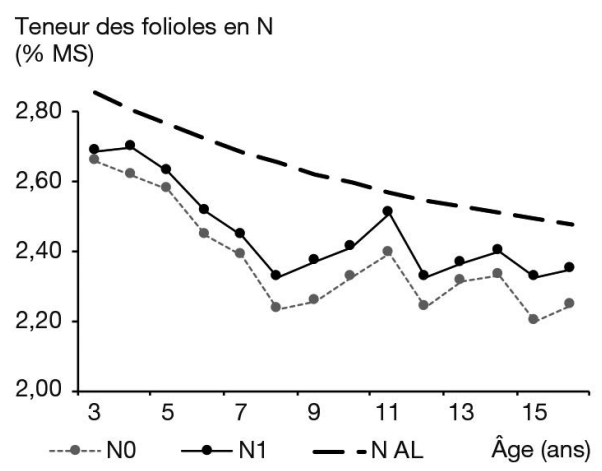

N0, sans engrais; N1, 3 kg d'urée / palmier / an

Figure 8. Effet de l'âge et de la fertilisation en azote sur les teneurs foliaires des palmiers dans la région de Quinindé en Équateur.

Les valeurs moyennes obtenues pour chaque modalité diminuent régulièrement avec l'âge. L'écart entre N1 et $\mathrm{N} 0$ a augmenté et est devenu significatif à partir de 8 ans mais il n'a pas été possible d'atteindre les valeurs prédites par le modèle N AL. 
En moyenne, $\mathrm{N} 1 / \mathrm{N}$ AL $=93 \%$ et $\mathrm{N} 0 / \mathrm{N} \mathrm{AL}=90 \%$.

Les apports d'urée n'ont pas d'effet positif sur le rendement, et l'on a conclu qu'il suffisait d'atteindre $90 \%$ du modèle pour que la nutrition azotée soit correcte dans cette plantation où l'ensoleillement annuel est faible.

\section{Variations interannuelles}

Les teneurs en $\mathrm{N}$ varient souvent d'une année à l'autre, à l'échelle d'une partie de la plantation dans la même tranche d'âge (figure 9), voire à l'échelle de toute une plantation. L'ensemble des teneurs en $\mathrm{N}$ diminue ou augmente d'une année à l'autre sans que la fertilisation ait été modifiée.

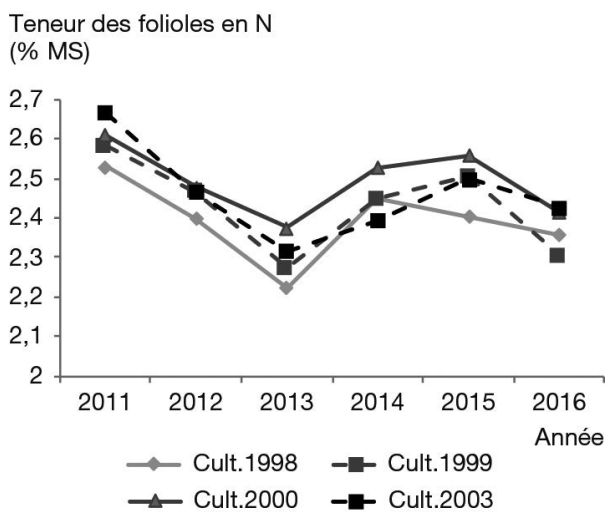

Figure 9. Exemple de synchronisme des variations des teneurs moyennes en $\mathrm{N}$, observés pour les cultures plantées de 1998 à 2003 en Colombie.

| Une baisse marquée des teneurs en 2013 est observée pour les quatre années de plantation.

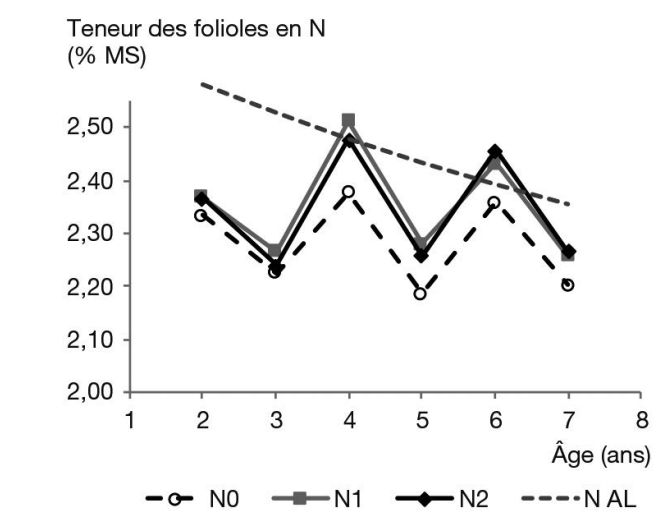

N0, sans engrais; N1, 1,6 kg/palmier/an; N2, 3,2 kg/palmier/an

Figure 10. Effets des modalités d'apport d'engrais et comparaison de ces effets aux variations interannuelles.

Dans cet essai les apports d'urée N1 et N2 augmentent significativement les teneurs par rapport au témoin N0 sans fertilisation. Cependant les variations interannuelles des teneurs moyennes en $\mathrm{N}$ sont largement supérieures à l'effet des engrais. 
Lorsque des variations interannuelles apparaissent dans un essai de fertilisation qui étudie l'azote (figure 10), on constate qu'elles sont indépendantes des quantités d'engrais appliquées et que leur amplitude peut être supérieure à l'effet des apports d'engrais azoté.

Ces phénomènes observables à l'échelle d'une plantation entière ne semblent donc pas liés aux apports d'engrais mais pourraient s'expliquer par les mécanismes physiologiques qui régulent l'allocation de $\mathrm{N}$ dans les folioles.

Lorsque les variations interannuelles sont supérieures à l'effet des engrais, déterminer un ratio optimal par rapport au modèle $\mathrm{N}$ AL demande plusieurs années d'observation.

\section{Conclusion}

En conclusion pour ce qui concerne la gestion de la nutrition azotée, il est préférable de ne pas utiliser directement les teneurs observées mais de tenir compte de l'âge des cultures et de déterminer les valeurs de référence par rapport à un modèle. Lorsque les teneurs atteignent ou dépassent les valeurs du modèle il n’y a généralement pas d'amélioration du rendement à attendre. Lorsque les teneurs sont inférieures au modèle, on déterminera selon le site jusqu’à quelle valeur ce déficit est acceptable.

\section{Phosphore (P)}

Les teneurs en $\mathrm{P}$ des folioles varient de 0,12 à $0,19 \%$ du poids en matière sèche (MS). Les essais de fertilisation indiquent que les teneurs foliaires répondent généralement bien aux apports de $\mathrm{P}$ et éventuellement se traduisent par une augmentation du rendement. Cependant, ces réponses ne permettent pas de déterminer une valeur seuil indicatrice d'une déficience en $\mathrm{P}$.

\section{Modèle d'équilibre entre les éléments $P$ et $N$}

Il est recommandé d'utiliser le modèle d'équilibre entre les éléments $\mathrm{P}$ et $\mathrm{N}$ établi par Ollagnier et Ochs en 1981 :

$$
\text { Pmod }=0,0487 \times \mathrm{N}+0,039
$$

Pmod est la teneur en P (\% MS) prédite en fonction de la teneur en N (\% MS).

Ce modèle traduit une relation très stable confirmée dans plusieurs pays d'Afrique, d'Asie et d'Amérique (figure 11). Sur le continent américain, il est applicable aussi pour les hybrides $\mathrm{O} \times \mathrm{G}$ (figure 12).

Lorsque le ratio $\mathrm{P} / \mathrm{Pmod}$ (rapport entre la teneur en $\mathrm{P}$ mesurée et la teneur en $\mathrm{P}$ prédite par le modèle Pmod) se rapproche de $100 \%$, il y a peu d'effet des apports d'engrais sur les rendements. Si P/Pmod se situe en dessous de 90\%, des apports de phosphate pour corriger les teneurs foliaires seront probablement suivis d'une amélioration du rendement. 


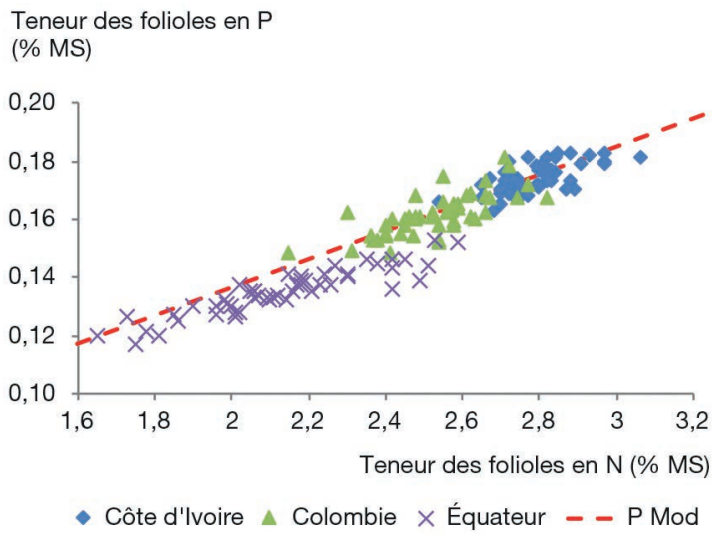

Figure 11. Teneurs en $\mathrm{N}$ et en $\mathrm{P}$ (\% MS) de palmeraies guineensis matures situées en Colombie, en Côte d'Ivoire et en Équateur.

En Équateur, les teneurs en $\mathrm{N}$ limitées par l'ensoleillement expliquent les faibles teneurs en P. À l'inverse, en Côte d'Ivoire, les conditions du milieu font que la nutrition en $\mathrm{N}$ est abondante, ce qui se répercute sur les teneurs en P. Cependant le modèle explique bien la relation entre $\mathrm{N}$ et $\mathrm{P}$ dans chaque situation. L'hypothèse retenue pour expliquer la constance de l'équation est que la composition des protéines du limbe varie peu.

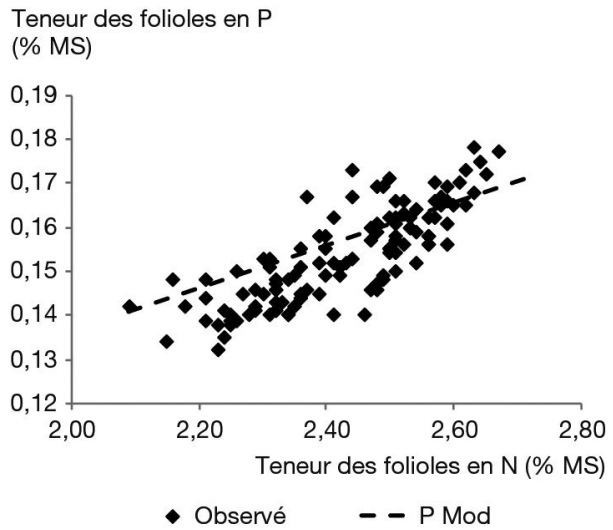

Figure 12. Relation entre les teneurs en $\mathrm{N}$ et en $\mathrm{P}$ pour le matériel hybride $\mathrm{O} \times \mathrm{G}$.

Dans cette plantation en Équateur, les teneurs en $\mathrm{N}$ observées sur des plants de 5 à 17 ans se répartissent selon un gradient de 2,1\% à 2,7\% MS. Le modèle explique la plus grande part de la variation des teneurs en $\mathrm{P}$ de 0,13 à $0,18 \%$ MS. Les échantillons de folioles les moins pourvus en P présentent un déficit de $12 \%$ par rapport à la valeur prédite par le modèle.

Cette relation entre $\mathrm{P}$ et $\mathrm{N}$ est importante, puisqu' elle permet de s'affranchir des facteurs qui font varier la nutrition en $\mathrm{N}$ et se répercutent sur les teneurs en $\mathrm{P}$ (figure 13). 

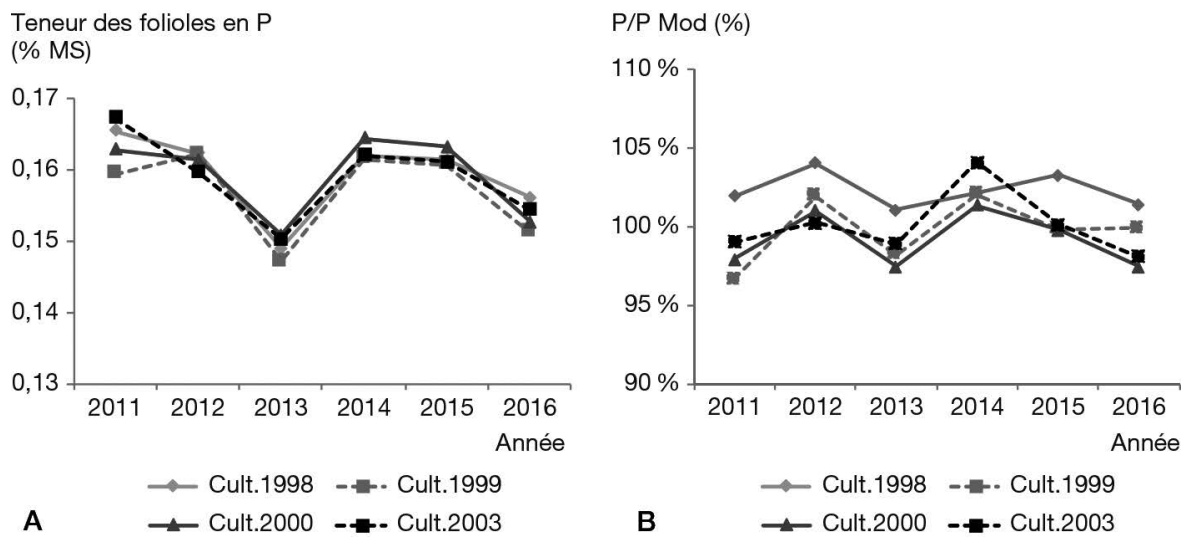

Figure 13. Variation interannuelle des teneurs en $\mathrm{P}$ pour les cultures plantées de 1998 à 2003 (figure 13A) et ses conséquences sur le ratio des teneurs P/PMod

( $P$ observé/P prédit par le modèle, figure 13B).

Dans l'exemple de la figure 9 des facteurs non contrôlés ont fait chuter les teneurs en $\mathrm{N}$ en 2013 et cela s'est traduit par une variation concomitante des teneurs en P (figure 13A), mais les variations du ratio $\mathrm{P} / \mathrm{PMod}$ sont atténuées (figure 13B). On ne peut donc pas conclure à une dégradation de la nutrition phosphorée en 2013 pour les quatre années de culture examinées. Lamplitude des variations interannuelles du ratio P/PMod est atténuée par rapport aux variations des teneurs en $\mathrm{P}$.

\section{Encadré 4. Interprétation des effets conjoints des engrais azotés et des engrais phosphorés sur les teneurs en $\mathrm{N}$ et en $\mathrm{P}$.}

Un exemple est fourni par les résultats d'un essai factoriel (figure 14) qui teste les apports de $\mathrm{P}$ sous forme de superphosphate triple (TSP) et de $\mathrm{N}$ sous forme d'urée :

- PO et N0 sont les modalités sans engrais azoté ou phosphoré;

- P1, 0,75 kg/palmier/an; P2, 1,5 kg/palmier/an;

- N1, 1,5 kg/palmier/an; N2, 3 kg/palmier/an.

L'essai a montré très rapidement que les rendements étaient limités par une forte déficience en $\mathrm{P}$, ce qui a été confirmé par les analyses de sol. La première dose de TSP (P1) est suffisante pour atteindre le rendement le plus élevé.

Teneur des folioles en $\mathrm{P}$

(\% MS)

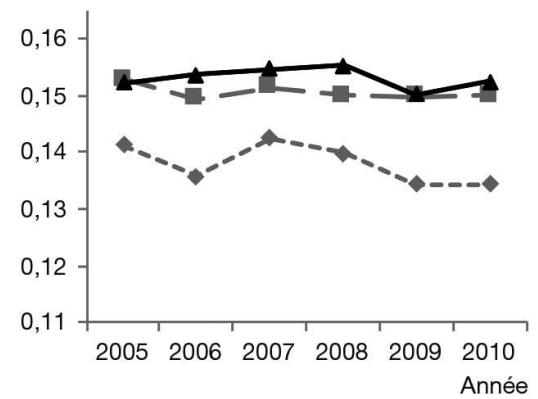

A

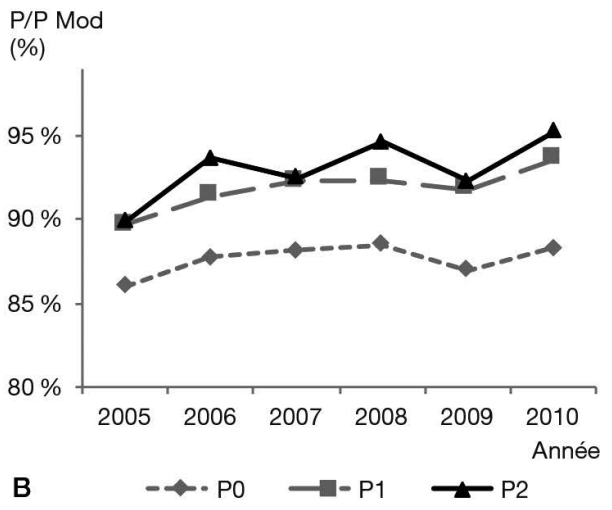

P/P Mod

$(\%)$

Figure 14. Réponses des teneurs foliaires en $P$ (figure 14A) et du ratio P/PMod (figure 14B) aux apports de TSP. 
L'effet du TSP sur la nutrition phosphorée apparaît dans la figure 14, ce qui se traduit par des augmentations significatives des teneurs en $P$ (figure 14A) et du ratio P/PMod (figure 14B) obtenus avec les apports P1 et P2. L'augmentation de rendement peut donc s'expliquer par une amélioration de la nutrition phosphorée.

Teneur des folioles en $\mathrm{N}$

(\% MS)

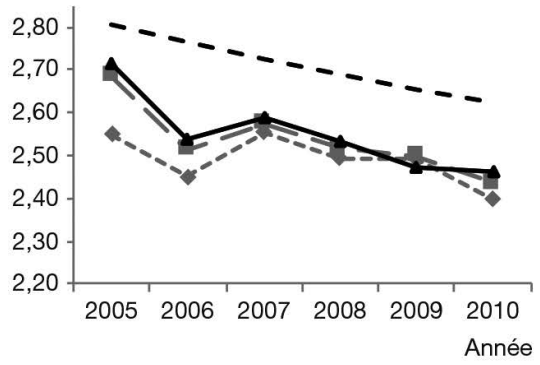

$A-\leftrightarrow \cdot N 0 \rightarrow-N 1 \longrightarrow N 2--N A L$

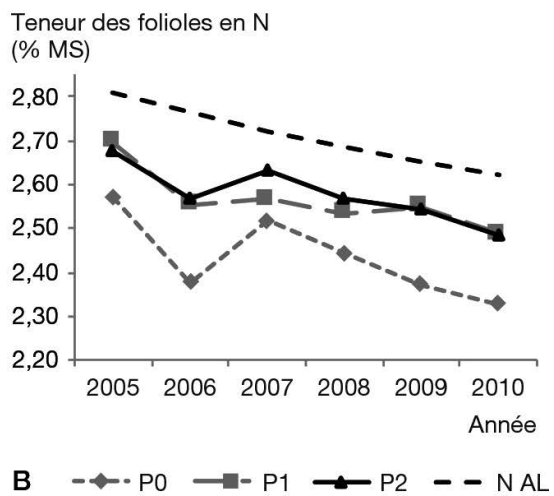

Figure 15. Réponses des teneurs foliaires en $N$ aux apports d'urée (figure 15A) et aux apports de TSP (figure 15B). N AL est la valeur prédite par le modèle qui décrit la variation des teneurs en $\mathrm{N}$ en fonction de l'âge des palmiers.

Les apports d'urée n'ont pas eu d'effet sur les rendements, ce que traduit l'absence de réponse des teneurs foliaires en $\mathrm{N}$. On a bien observé un effet de l'urée sur les teneurs en $\mathrm{N}$ en 2005 à 4 ans mais cet effet a disparu par la suite, bien que les teneurs soient très inférieures aux valeurs du modèle $\mathrm{N} A \mathrm{~L}$ Cependant pour statuer sur la nutrition azotée il faut prendre en compte la figure 15B qui montre que les teneurs en $\mathrm{N}$ augmentent significativement avec les apports de TSP. Cet engrais réduit l'écart par rapport au modèle $\mathrm{N} A \mathrm{~L}$.

En définitive, dans ces sols très faiblement pourvu en $\mathrm{P}$, les apports de TSP améliorent à la fois la nutrition en $\mathrm{P}$ et en $\mathrm{N}$. Ces résultats permettent de recommander que le ratio $\mathrm{P} / \mathrm{PMod}$ atteigne $90 \%$. Ils confirment aussi que la nutrition azotée est correcte avec un ratio N/N AL de $95 \%$ ou plus, obtenu après avoir corrigé la déficience en $P$.

\section{Potassium (K)}

Le potassium est l'élément clef de la nutrition du palmier à huile et il a joué un rôle important comme outil de pilotage pour la mise au point du diagnostic foliaire (DF) dès 1950. Toutefois, chaque situation exige d'adapter ses propres normes : définir une plage de teneurs optimales en potassium (K) est la principale difficulté que rencontrent les agronomes en charge de la fertilisation du palmier à huile.

Dans chaque situation, il faut déterminer la teneur foliaire optimale en K. Dans les travaux originaux, les teneurs foliaires en $\mathrm{K}$ ont souvent été considérées comme optimales (Ollagnier et Ochs, 1981) lorsqu'elles dépassaient 1\% du poids en matière sèche (MS). Mais, par la suite, on a montré que des économies d'engrais pouvaient être réalisées en ajustant les teneurs optimales à chaque situation, afin de tenir compte de la nature des sols, du climat et des rendements potentiels. Des teneurs optimales comprises entre $0,80 \%$ et $1,0 \% \mathrm{MS}$ ont alors été publiées pour E. guineensis selon les conditions du milieu (Caliman et al., 1994). Pour Goh and Härdter (2003), des teneurs «normales» se situent entre 0,90 et 1,30\% pour la plupart des sols. 
Le climat, le matériel végétal et le sol sont des facteurs indépendants des pratiques de fertilisation qui influencent aussi les teneurs foliaires optimales en K.

\section{Influence du climat}

Une saison sèche plus ou moins marquée a des conséquences sur la teneur foliaire en K. Pour Ollagnier et al. (1987) cité par Caliman et al. (1994), la teneur optimale en $\mathrm{K}$ passe par un maximum pour des déficits hydriques annuels moyens proches de $200 \mathrm{~mm}$ (figure 16). Cette relation est fondée essentiellement sur des données recueillies en Afrique et elle n'est sans doute pas applicable à d'autres continents.

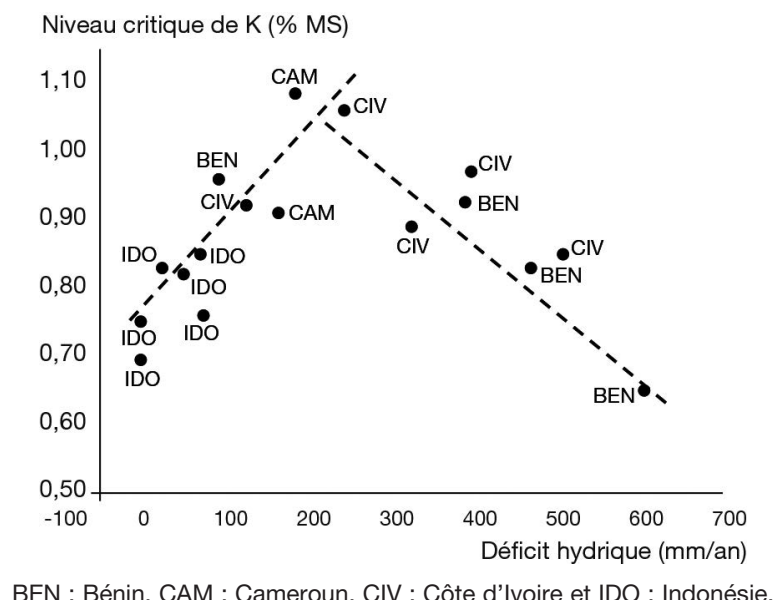

Figure 16. Relation entre la teneur en $\mathrm{K}$ optimale (niveau critique) et le déficit hydrique annuel en Afrique et en Indonésie (d'après Caliman et al., 1994).

\section{Influence du matériel végétal}

L'origine génétique du matériel végétal est aussi un paramètre important (Tan et Rajaratnam, 1978; Jacquemard et al., 2009). La principale difficulté pour prendre en compte ce facteur est actuellement la difficulté à établir ou à prédire les teneurs optimales propres à chaque origine génétique. Il est possible de détecter expérimentalement des différences de teneurs pour des croisements recevant la même fertilisation (figure 17) mais on ignore quelles sont leurs conséquences sur les besoins en nutriments.

\section{Influence de la nature des sols}

L'abondance en calcium $(\mathrm{Ca})$ échangeable dans le sol tend à diminuer les teneurs foliaires en $\mathrm{K}$. Ce phénomène que l'on observe lorsqu'on applique des chlorures s'explique par une allocation préférentielle du calcium dans la foliole, au détriment du potassium. Le mécanisme est décrit section «Tenir compte des teneurs du sol en calcium ", page 65. On retiendra que sur les sols riches en calcium, les applications de $\mathrm{KCl}$ diminuent la teneur foliaire en $\mathrm{K}$ jusquà environ 0,80\% $\mathrm{MS}$. Ces teneurs sont faibles par rapport aux valeurs de référence mais généralement ces déficiences n'ont pas d'impact sur le rendement. 


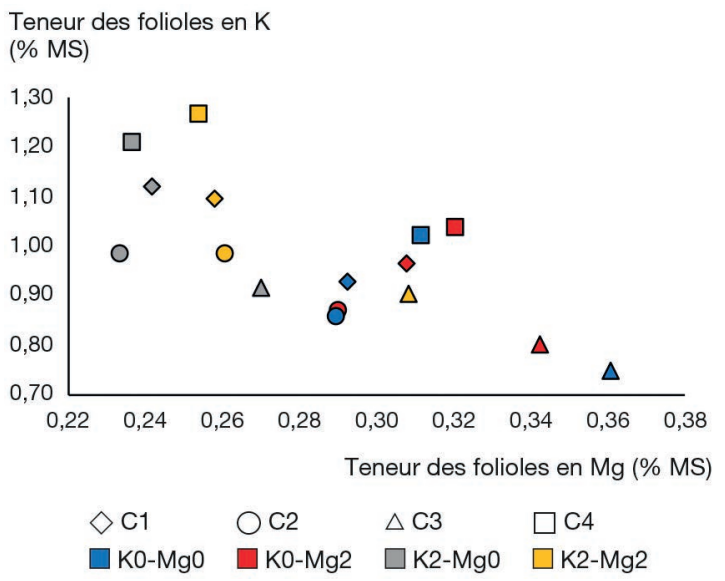

Cette expérience de nutrition minérale croise 3 doses de $\mathrm{KCl}(\mathrm{K} 0, \mathrm{~K} 1, \mathrm{~K} 2)$ et 3 doses de kiesérite (Mg0, Mg1, Mg2), et étudie 4 croisements connus $(C 1, C 2, C 3, C 4)$ pour leurs teneurs foliaires en $\mathrm{K}$ et Mg contrastées. On a examiné l'effet des doses extrêmes (0, sans engrais; 1 , dose «intermédiaire" pour $\mathrm{KCl}$ et Kiesérite; 2 : dose maximale de $\mathrm{KCl}$ ou de kiesérite) pour tester les croisements après quatre années d'application du protocole.

Figure 17. Test de la spécificité du matériel végétal.

Les résultats confirment la spécificité des croisements : $\mathrm{C} 3$ et $\mathrm{C} 4$ ont des teneurs foliaires élevées, respectivement en $\mathrm{Mg}$ et $\mathrm{K}, \mathrm{C} 2$ présente des teneurs faibles en $\mathrm{Mg}$ et en $\mathrm{K}$ (d'après Dassou et al., 2018).

\section{Calcium (Ca)}

En plantations matures de 12 ans et plus, les teneurs foliaires en calcium sont presque toujours comprises entre $0,50 \%$ et $0,80 \%$ du poids en matière sèche (MS). Elles peuvent se rapprocher de $1 \%$ dans les situations où l'on rencontre cet élément en abondance dans le sol.

Lobservation des teneurs foliaires en Ca aide à comprendre les dysfonctionnements observés avec d'autres cations, en particulier $\mathrm{K}$ et parfois $\mathrm{Mg}$. Les apports de $\mathrm{Ca}$ (application de phosphates, amendements avec de la chaux ou du gypse) peuvent diminuer les teneurs en $\mathrm{K}$ et en $\mathrm{Mg}$. Il est aussi intéressant d'analyser la relation entre les teneurs en $\mathrm{Ca}$ et en $\mathrm{Cl}$ dans les dispositifs expérimentaux comme cela est expliqué à la section "Tenir compte des teneurs du sol en calcium», page 65.

Il est toutefois impossible de déterminer une teneur foliaire optimale en calcium, car il n'y a pas de symptômes de déficience connus. Il n'y a pas non plus de réponse des rendements à l'apport d'engrais calcique même si le chaulage, pratiqué pour améliorer la structure du sol, peut entraîner une augmentation des rendements lorsque cela se traduit par une meilleure alimentation hydrique.

\section{Magnésium (Mg)}

Les teneurs foliaires en $\mathrm{Mg}$ varient de moins de $0,10 \%$ du poids en matière sèche (MS), en cas de forte déficience, à des teneurs comprises entre 0,30 et $0,40 \% \mathrm{MS}$ 
lorsque le sol est bien pourvu. Dans un essai de fertilisation bien contrôlé, on observe une baisse significative de rendement à partir d'une une concentration inférieure à 0,16\% (Dubos et al., 1999), alors que les symptômes sur les feuilles se manifestent dès la concentration de $0,20 \%$.

La déficience en $\mathrm{Mg}$ est souvent plus spectaculaire que grave. Pour Webb et al. (2009), le magnésium est rapidement relocalisé à partir des tissus les plus anciens, où les symptômes apparaissent en premier et deviennent les plus intenses, vers les tissus plus jeunes. Les symptômes sont plus intenses dans les parties du feuillage les plus exposées à la lumière, comme signalé à la section "Peut-on se baser sur des symptômes de déficience...?» page 14. Pour cette raison les palmiers plantés en bordure de parcelle paraissent plus fréquemment déficients.

\section{Effet du site}

Une teneur foliaire en $\mathrm{Mg}$ supérieure ou égale à $0,24 \% \mathrm{MS}$ a souvent été considérée comme correcte. Il est toutefois probable que ce seuil soit surévalué (Ollagnier et Ochs, 1981) par rapport à l'impact de cet élément sur la production.

Au Pérou, malgré la présence de quelques symptômes de déficience, des teneurs comprises entre 0,18 et $0,22 \%$ MS ont été considérées comme suffisantes, car les rendements n'augmentaient pas avec des teneurs plus élevées.

Dans la région de Quinindé (Équateur), où l'insolation est faible, les teneurs foliaires en $\mathrm{Mg}$, comme celles en azote, sont plus faibles que sur d'autres sites pour le même type de matériel végétal et une fertilisation équivalente (figure 18). Dans cette région, des teneurs en $\mathrm{Mg}$ comprises entre $0,16 \%$ et $0,20 \% \mathrm{MS}$ sont suffisantes pour du matériel E. guineensis d'origine Deli $\times$ La Mé.

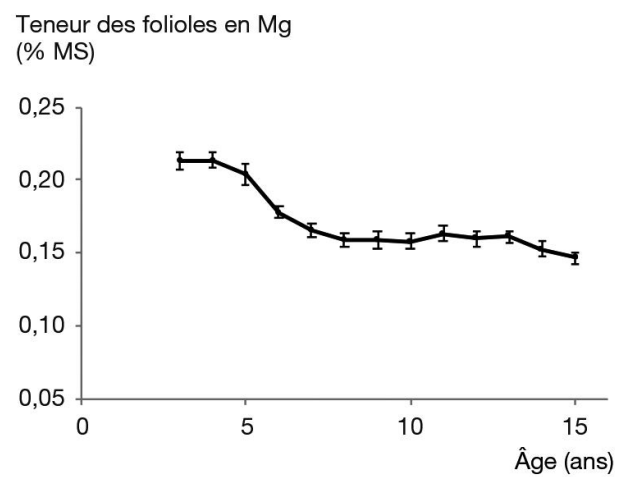

Figure 18. Teneurs foliaires moyennes en $\mathrm{Mg}$ en fonction de l'âge, dans la région de Quinindé (Equateur), où l'insolation est faible.

La baisse observée entre 3 et 7 ans est due à l'augmentation de la biomasse des feuilles. Elle est similaire à ce que l'on observe dans d'autres circonstances, mais les teneurs moyennes se stabilisent aux alentours de $0,16 \%$ MS alors que les apports d'engrais devraient les maintenir au-delà de 0,20\% MS. Les essais de fertilisation ont confirmé que ni les teneurs foliaires ni les rendements n'augmentaient significativement avec les apports d'engrais magnésiens et que ces absences de réponses se présentaient pour les différents types de sol de la plantation. 


\section{Origine du matériel végétal}

Lorigine du matériel végétal exerce une influence sur les teneurs foliaires en $\mathrm{Mg}$. En effet, le matériel E. guineensis Deli $\times$ Yangambi présente des teneurs en $\mathrm{Mg}$ plus élevées d'environ 0,04\% MS que E. guineensis Deli $\times$ La Mé (F. Corrado, comm. pers.). Des progrès sont attendus avec les résultats des dispositifs spécifiques mis en place par le Cirad et ses partenaires pour étudier l'effet du matériel végétal sur les teneurs en $\mathrm{K}$ et en $\mathrm{Mg}$.

En Amérique latine où le matériel hybride $\mathrm{O} \times \mathrm{G}$ est maintenant très répandu pour des raisons sanitaires, on a observé une forte variabilité d'un croisement à l'autre. Ces différences apparaissent dans la couleur du feuillage observée au champ (photo 7) mais aussi par des différences de teneurs foliaires en $\mathrm{Mg}$ avec une fertilisation identique (figure 19). L'interprétation des résultats d'analyse est parfois délicate mais des travaux permettront, on l'espère, de préciser les normes en fonction des origines.

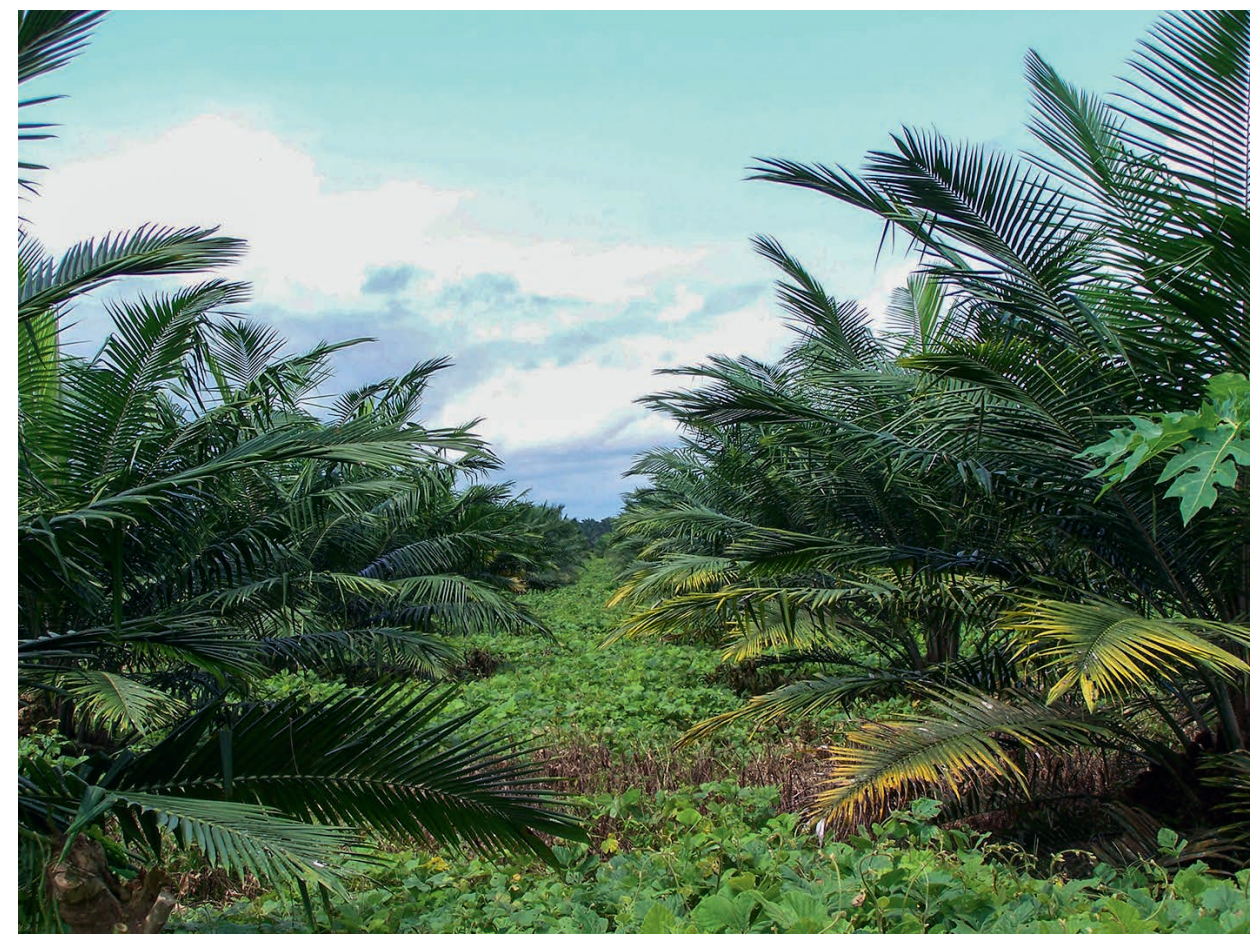

Photo 7. Spécificité de l'origine génétique du matériel $O \times G$

vis-à-vis de la nutrition magnésienne : comparaison de deux croisements hybrides d’origine Coari $\times$ La Mé plantés côte à côte dans la même parcelle et ayant reçu la même fertilisation jusqu'à 4 ans.

Les palmiers du rang de droite (descendance TT3314) expriment des symptômes intenses alors que ceux du rang de gauche (descendance TT3306) sont indemnes de jaunissement, ce qui exprime la spécificité de l'origine génétique du matériel $\mathrm{O} \times \mathrm{G}$ vis-à-vis de la nutrition magnésienne. 


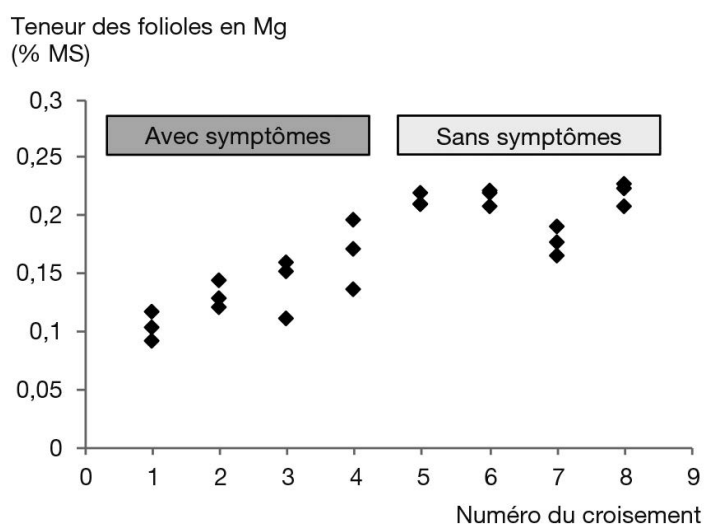

Figure 19. Influence du matériel végétal (croisements 1 à 4 ayant en commun le même pollen $E$. guineensis, croisements 5 à 8 issus d'un autre pollen) sur la nutrition en magnésium. Essai conduit en Équateur.

À 5 ans les croisements 1 à 4 présentent des teneurs en Mg inférieures à celles des croisements 5 à 8 . Les symptômes de déficience sont également beaucoup prononcés pour le premier groupe.

\section{Chlore $(\mathrm{Cl})$}

Les teneurs foliaires en chlore sont généralement satisfaisantes, car on utilise du chlorure de potassium $(\mathrm{KCl})$ en fertilisation, c'est une pratique courante pour couvrir les besoins en $\mathrm{K}$.

La déficience en $\mathrm{Cl}$ existe dans les zones éloignées des océans où les apports atmosphériques sont inexistants. C'est le cas du bassin amazonien (Pérou, Équateur, Brésil, Colombie) mais aussi de la vallée du Magdalena en Colombie où la déficience a été mise en évidence par Ollagnier et Ochs (1971).

Les teneurs foliaires sont correctes à partir de $0,50 \%$ du poids en matière sèche (MS). Ce seuil est rapidement atteint à l'entrée en production avec l'application de $\mathrm{KCl}$. En culture adulte, les apports pour satisfaire la demande en $\mathrm{K}$ sont toujours suffisants pour satisfaire simultanément la demande en $\mathrm{Cl}$ et les teneurs foliaires en $\mathrm{Cl}$ sont comprises entre 0,60 et $1 \%$ MS.

Il est recommandé d'analyser le chlore chaque année si la palmeraie est située dans une zone à déficience édaphique. Dans les autres situations, quelques contrôles périodiques sont utiles à l'entrée en production à 3 et 4 ans.

Dans les essais de fertilisation qui utilisent $\mathrm{du} \mathrm{KCl}$, il faut aussi analyser le chlore pour interpréter correctement les effets du $\mathrm{KCl}$ sur le rendement.

\section{Soufre (S)}

Les cas de déficience avérée en soufre qui se traduisent par une baisse de rendement sont rares. Les teneurs foliaires en $\mathrm{S}$ et en $\mathrm{N}$ sont fortement corrélées et les symptômes décrits en jeune culture sont similaires à ceux de la déficience en $\mathrm{N}$. 
Ollagnier et Ochs (1972) ont considéré que le niveau critique des teneurs en soufre était compris entre 0,20 et $0,23 \%$ du poids en matière sèche (MS). Plus récemment, Gerendás et al. (2009) ont ramené ce seuil critique à 0,15\%.

Lanalyse foliaire du soufre n'est pas une pratique standard dans le contrôle de la nutrition des plantations de palmier à huile, sans doute en raison du coût additionnel de la mesure et du peu d'incidence de cet élément sur la productivité. Afin d'assurer un apport en soufre, il est recommandé qu'au moins une des sources d'engrais soit un sulfate, par exemple du sulfate d'ammonium [ $\left.\left(\mathrm{NH}_{4}\right) 2 \mathrm{SO}_{4}\right]$ pour les besoins en $\mathrm{N}$ ou de la kiesérite (sulfate de magnésium totalement soluble) pour les besoins en $\mathrm{Mg}$.

\section{Oligo-éléments : bore, cuivre, fer, manganèse}

\section{Cas du bore (B)}

Parmi les éléments dits mineurs, le bore (B) est le plus analysé en routine et il fait l'objet d'apports d'engrais. Le diagnostic de la déficience en bore et la gestion des apports restent controversés pour différentes raisons.

Les symptômes de déficience sont liés à un dysfonctionnement de l'apex végétatif des plants qui se traduit par des malformations plus ou moins intenses des tissus foliaires produits par l'apex (voir page 17). Les véritables déficiences sont plus fréquemment observées entre 2 et 5 ans et réduisent sensiblement la surface foliaire. Il en résulte une baisse de l'activité photosynthétique et de la productivité qui seront efficacement corrigées par des apports de borax. Les véritables déficiences sont plus rares en phase mature hormis sur certains sols intrinsèquement très pauvres comme on en rencontre en Amazonie. Lorsque la surface foliaire n'est pas réduite, les symptômes de bandes blanches ou la malformation des folioles terminales ne renseignent pas sur la présence réelle d'une déficience, car ces symptômes peuvent avoir d'autres origines; ils peuvent même être plus fréquents lorsqu'on applique de l'engrais (figure 20) : il faut être très prudent avant de conclure à une déficience en bore par l'observation de symptômes isolés dans les cultures matures.

L'analyse des teneurs en bore en ppm du poids en matière sèche de la feuille 17 ne fournit pas d'information utile. D'une part, la détermination de $B$ en laboratoire est peu précise avec un intervalle de confiance de \pm 2 à 3 ppm. D'autre part, les teneurs en B varient de 10 à $30 \mathrm{ppm}$, voire jusqu'à $50 \mathrm{ppm}$ peu de temps après un apport. On lit souvent qu'elles doivent être supérieures à $12 \mathrm{ppm}$, mais aucun résultat expérimental ne prouve que cette valeur corresponde à un seuil de déficience. On ne note pas de de réduction de la surface foliaire en relation avec des teneurs inférieures à $12 \mathrm{ppm}$. À l'inverse, on observe de jeunes palmiers avec des feuilles courtes alors que les teneurs des feuilles 17 sont correctes. Cette absence de relation entre l'état végétatif et les teneurs foliaires est due au fait qu'à la différence des autres éléments, le bore est un élément particulièrement peu mobile. La feuille de rang 17 est probablement inadaptée pour observer une déficience à un moment donné car elle a été émise 6 à 8 mois avant la date à laquelle on dispose des résultats de l'analyse. Une teneur élevée ne traduit donc pas une réserve potentiellement recyclable vers les feuilles en cours d'épanouissement où s'exprimera la déficience. 


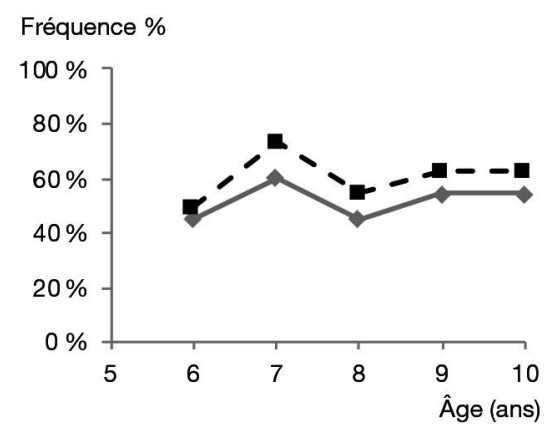

A

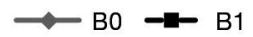

Fréquence \%

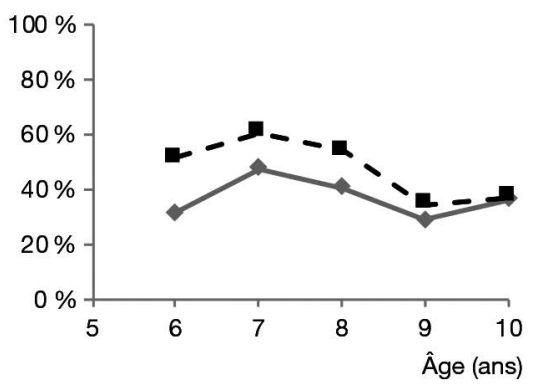

B

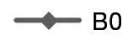

B1

B0, témoin non fertilisé; B1, $100 \mathrm{~g}$ de borax (15\% B) /palmier/an

Figure 20. Essai de fertilisation en bore entre 6 et 10 ans en Côte d'Ivoire : pourcentage de palmiers présentant des bandes blanches (figure 20A) et des malformations des folioles terminales (figure 20B).

De 7 à 10 ans, la présence de bandes blanches est significativement plus fréquente avec l'apport de borax sans que cet effet soit expliqué. Jusqu'à 8 ans les malformations du limbe des folioles sont plus fréquentes pour les palmiers qui reçoivent du bore même si cela n'est pas statistiquement significatif. Les apports de borate n'ont pas eu d'effet sur les rendements observés ce qui confirme qu'il n'y a pas de déficience et que les symptômes présents ne sont pas pertinents.

\section{Cas du cuivre (Cu)}

Le niveau critique en cuivre est de 3 ppm, au-dessus de ce seuil, il n'y a quasiment aucun risque de déficience. Cependant, au-dessous de 2 ppm, des déficiences peuvent apparaître. Comme la précision des analyses en cuivre est de l'ordre de $1 \mathrm{ppm}$, il reste difficile d'utiliser le seul résultat de laboratoire pour décider d'appliquer ou non du sulfate de cuivre. La surveillance des symptômes au champ reste donc nécessaire. Sur tourbe, l'application de sulfate de cuivre $\left(\mathrm{CuSO}_{4}\right)$ à la surface du sol est une mesure préventive efficace dans des blocs où la déficience en cuivre est attestée. Quatre applications sont échelonnées à partir de la plantation jusqu'à 18 mois, et il est rare qu'une correction soit encore nécessaire après trois ans. On peut aussi utiliser le sulfate de cuivre à titre curatif, en fractionnant les applications pour éviter l'immobilisation du produit dans des complexes organiques.

\section{Cas du fer (Fe)}

La déficience en fer $(\mathrm{Fe})$ existe sur tourbe mais elle est très temporaire : elle se manifeste en première année de plantation; elle est généralement non létale, les palmiers retrouvent une végétation normale sans nécessiter de correction. La déficience en fer se caractérise par des alternances de bandes vertes et jaunes alignées le long du limbe (interveinal chlorosis).

\section{Cas du manganèse (Mn)}

Les teneurs foliaires en manganèse $(\mathrm{Mn})$ varient largement entre 100 et 600 ppm sans qu'il soit possible d'établir, avec certitude, un niveau critique lorsque les palmiers ne 
présentent pas de déficience. Les palmiers atteints peuvent être traités individuellement par des applications de sulfate de manganèse jusqu’à récupération du feuillage.

\section{Interpréter les teneurs foliaires en tenant compte des caractéristiques propres à la plantation}

Les résultats des analyses foliaires doivent nous permettre de porter un diagnostic sur l'état de la nutrition à un instant donné (déficience, teneur correcte, teneur très élevée) et d'en déduire une recommandation de fertilisation (dose de redressement, de maintien, arrêt des apports). Les exemples ci-dessus montrent qu'il est indispensable de connaitre le contexte dans lequel ont été obtenus ces résultats. En fonction de l'âge, du matériel végétal, des propriétés des sols, du climat, telle teneur sera considérée comme correcte et ne nécessitera qu'un ajustement modeste ou, au contraire, elle appellera une correction plus forte.

De plus, une part de la variabilité constatée dans les résultats obtenus peut être due au laboratoire d'analyse des tissus végétaux (et d'analyse des sols pour la CEC et les cations échangeables), en fonction de ses équipements, ses méthodes d'analyse (plusieurs méthodes pour $\mathrm{N}, \mathrm{P}$, plusieurs méthodes de calcination, etc.) et son fonctionnement (fréquence et précision des protocoles d'auto-contrôle, certification, analyses croisées avec d'autres laboratoires). Le choix d'un laboratoire capable de fournir des analyses minérales de grande qualité avec un degré de précision stable dans le temps est fondamental.

En conclusion, l'interprétation des teneurs foliaires en éléments minéraux, est un exercice délicat qui demande de l'expérience et doit prendre en compte les caractéristiques propres à chaque plantation car ces dernières vont agir sur les teneurs optimales de référence. C'est pourquoi on recommande de coupler le diagnostic foliaire avec la mise en place d'un ou plusieurs essais de fertilisation qui vont permettre d'améliorer l'interprétation des résultats en fonction des apports d'engrais et des réponses du rendement. Cette démarche expérimentale in situ est expliquée page 50 (section "Principes des essais de fertilisation») et nécessite plusieurs années d'investigation. 



\section{2 \\ Échantillonner la plantation pour le suivi continu de la nutrition minérale}

Les teneurs foliaires ne réagissent pas seulement aux apports d'engrais; elles sont aussi influencées par d'autres facteurs (âge, matériel végétal, climat, sol). Avant de les comparer à des valeurs de référence adaptées pour chaque site (voir «Déterminer la plage des teneurs optimales" page 56), il faut pouvoir disposer périodiquement de résultats d'analyse pour toutes les cultures de la plantation.

Une plantation n'est pas un milieu homogène il faut donc multiplier les analyses pour tenir compte des changements dans l'espace, de l'âge, du matériel végétal et du sol. Pour des raisons pratiques et économiques il y a une limite au nombre d'échantillons qu'il est possible d'analyser chaque année pour une plantation donnée. Il faut donc structurer la plantation en petites unités de fertilisation les plus homogènes possible. L'objectif est de garantir que les résultats des analyses traduisent les effets des recommandations d'engrais que l'on a appliqué et que les analyses annuelles couvrent correctement l'ensemble de la plantation. Les normes pour la création des unités de fertilisation, la sélection des palmiers qui constitueront les échantillons, les techniques de prélèvement doivent être observées rigoureusement pour disposer d'un outil de pilotage précis.

Pour chaque plantation de palmier à huile, les expérimentations ou les conseils externes vont permettre de retenir des valeurs, considérées comme optimales, pour les teneurs en éléments minéraux. En parallèle on va déployer un outil de pilotage qui a pour but d'atteindre les valeurs optimales dans toute la plantation, en analysant des échantillons foliaires qui couvrent l'ensemble des cultures.

Le principe consiste à "découper» la plantation en petites unités de surface qui sont associées chacune à un échantillon foliaire de référence. Les échantillons foliaires sont prélevés chaque année et, en fonction des résultats de l'analyse, les recommandations de fertilisation de chaque unité sont établies pour l'année suivante. Ces unités de surface sont appelées des «unités de fertilisation» (UDF).

\section{Découper la plantation en plusieurs unités de fertilisation}

Les unités de fertilisation sont des unités de travail délimitées par des bordures faciles à observer, généralement des pistes. L'unité de fertilisation est constituée 
d'une ou de plusieurs parcelles contiguës. Cette disposition facilite le contrôle de l'épandage des engrais et garantit que tous les palmiers de l'unité soient traités de la même manière. À l'échelle des parcelles sont acquises d'autres données, comme les rendements et les relevés de maladies et on pourra agréger ces données à l'échelle de l'unité de fertilisation. On pourra croiser ces informations avec les teneurs foliaires (encadré 5).

Chaque unité de fertilisation doit être constituée d'une population homogène de palmiers (matériel végétal, année de plantation) cultivée dans des conditions homogènes (précédent cultural, préparation avant plantation, techniques culturales, nature des sols et topographie). Si cette exigence peut facilement être maitrisée pour les caractéristiques génétiques et les pratiques culturales, il n'en va pas de même pour le sol et la topographie, dont les hétérogénéités peuvent être détectées tardivement. Dans une plantation idéale où ces informations seraient cartographiées, la création des pistes et la délimitation des parcelles pourraient être pensées pour favoriser l'homogénéité interne de chaque unité de fertilisation. En réalité, les plantations sont presque toujours structurées en parcelles selon un maillage régulier avec des pistes de collecte parallèles orientées nord-sud et est-ouest. C'est une fois ce maillage établi que les unités de sol au sein des parcelles sont cartographiées.

La taille des unités de fertilisation est variable : la norme est en général de 50 ha et le minimum est rarement inférieur à 30 ha. La surface moyenne représentée par un échantillon foliaire est un compromis qui dépend d'autres spécificités de la plantation: nombre d'échantillons qu'il est possible de faire analyser en fonction du calendrier fixé, variabilité des propriétés des sols et de la topographie des parcelles, et homogénéité des opérations agricoles pour la préparation des parcelles. Dans des conditions très homogènes de sol et de topographie, certaines unités de fertilisation peuvent atteindre ou dépasser 100 ha. Lorsque les sols et la topographie font apparaître des discontinuités importantes, on peut réduire la taille des unités de fertilisation qui ne seront constituées que d'une seule parcelle (en général de 20 à 30 ha).

\section{Encadré 5. Prévoir d'alimenter des bases de données robustes dans le temps.}

Comme pour beaucoup de cultures pérennes, les données acquises dans les plantations de palmier à huile sont examinées en regroupant plusieurs années de mesure, jusqu'à une décennie et en combinant plusieurs variables disponibles à des échelles différentes (exemple : rendements à la parcelle, analyses foliaires par unité de fertilisation et analyses chimiques par unités de sol). Sur des pas de temps longs, la structure de la plantation subira des changements en particulier lors des replantations. L'organisation des données doit permettre de pouvoir les analyser rapidement sans risques, ce qui signifie que chaque objet permettant l'acquisition d'une valeur à un moment donné doit être identifié de façon unique et ses propriétés doivent être décrites (métadonnées). C'est le cas des unités de fertilisation, immatriculées par un numéro unique et décrite par la liste des palmiers des parcelles qui servent à prélever l'échantillon de l'unité de fertilisation. Avec cette information, on pourra examiner les relations entre les teneurs foliaires et d'autres variables collectées à l'échelle de la parcelle comme le rendement, le matériel végétal etc. Ces informations doivent être centralisées dans une base de données et partagées pour pouvoir résister au renouvellement du personnel qui collecte et analyse les données. 


\section{Prévoir le calendrier des prélèvements foliaires}

Le prélèvement des échantillons foliaires s'inscrit dans le calendrier agricole de la plantation. Pour des raisons de répétabilité, la période définie comme la plus favorable doit être respectée d'une année à l'autre. Le choix de cette période dépend de plusieurs facteurs (figure 21) :

- la disponibilité des équipes spécialisées dans cette tâche;

- les conditions météorologiques, les prélèvements doivent être effectués au cours d'une période peu pluvieuse;

- le cycle de production des cultures adultes. Dans des conditions climatiques données la productivité n'est pas répartie également tout au long de l'année. Lorsqu'un pic de récolte se répète tous les ans à une certaine époque, il faut programmer les prélèvements avant ou après ce pic;

- les délais de chaque activité, c'est-à-dire le temps nécessaire à l'obtention des résultats d'analyse par le laboratoire, les délais pour l'élaboration du programme de fertilisation, les achats des engrais, et leur approvisionnement. Ces délais doivent être compatibles avec les périodes optimales d'épandage.

Chaque plantation doit avoir son propre calendrier, déterminé avec beaucoup de réflexion et de retours d'expérience afin qu'il soit opérationnel. L'objectif est que le programme de fertilisation défini après une campagne de diagnostic foliaire soit exécuté deux mois avant les prélèvements suivants.

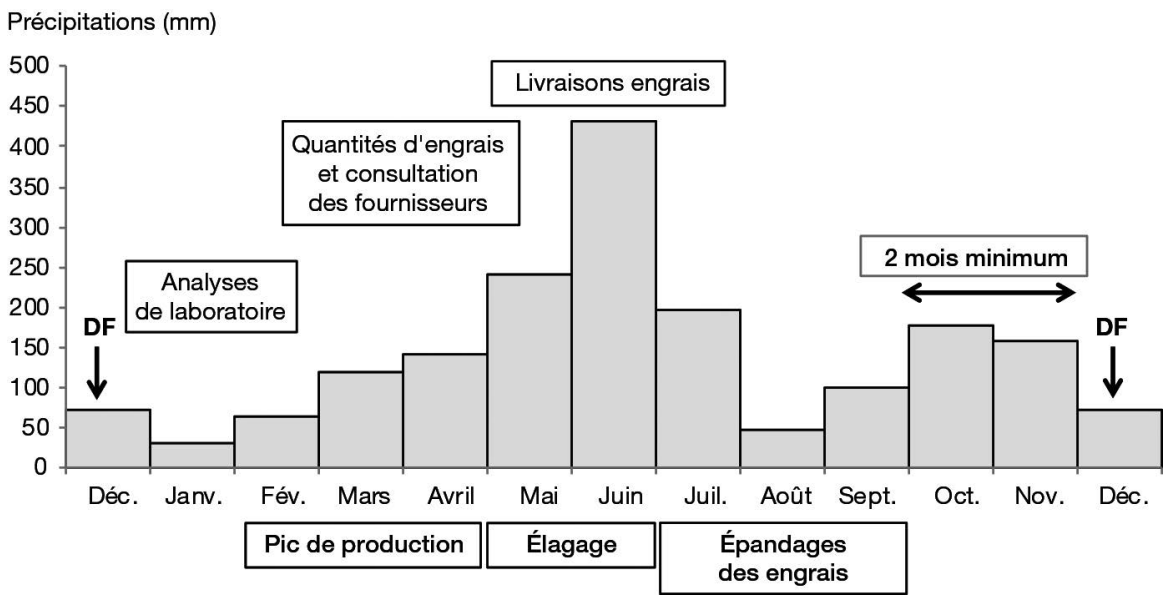

Figure 21. Exemple de calendrier opérationnel pour la fertilisation des plantations adultes sur un cycle d'un an.

Le prélèvement des échantillons foliaires (en décembre, noté "DF" sur la figure) et l'épandage des engrais (juillet à septembre) ont été programmés sur des périodes peu pluvieuses et lorsque la main-d'œuvre est peu mobilisée par d'autres activités essentielles comme la récolte et l'élagage des palmiers.

Il y a toujours des imprévus et le programme d'application des engrais peut prendre du retard. Quel que soit ce retard, les prélèvements des échantillons foliaires doivent 
être maintenus à la date prévue parce que l'analyse foliaire fournit un point de référence annuel dans un environnement physiologique normalisé et qu'il est important de pouvoir comparer les états nutritionnels d'une année à l'autre. Il faut aussi éviter de répercuter le retard sur la campagne suivante.

\section{Choisir les palmiers pour l'échantillon foliaire de référence au sein de l'unité de fertilisation}

Des critères d'apparence visuelle permettent de choisir les palmiers qui constitueront l'échantillon foliaire de référence de chaque unité de fertilisation. Individuellement, ils doivent être dans les normes de développement végétatif, être productifs et être entourés de palmiers aux caractéristiques similaires.

La localisation des palmiers de l'échantillon au sein de l'unité de fertilisation est le second critère important car l'objectif principal du diagnostic foliaire de référence est de prendre les meilleures décisions pour la fertilisation de chaque unité de fertilisation en vue d'optimiser leurs productivités (encadré 6).

\section{Restreindre l'échantillonnage de référence à une partie homogène de l'unité de fertilisation}

Pour prendre la meilleure décision pour une unité de fertilisation, il est préférable de restreindre l'échantillonnage de référence à une partie homogène de l'unité de fertilisation qui contribue de façon décisive au rendement de toute l'unité. L'échantillonnage est ciblé sur un faciès pour obtenir la recommandation la plus exacte possible pour cette partie de l'unité de fertilisation. Plusieurs principes peuvent aider à choisir une partie plutôt qu'une autre :

- lorsque l'unité de fertilisation est assez homogène et que seule une surface réduite présente une singularité due à la nature du sol (voir l'exemple encadré 6), il faut prélever sur la partie la plus vaste. Dans cet exemple, c'est la partie saine en $\mathrm{Mg}$ ( $85 \%$ de la surface). Ainsi, la partie déficiente en $\mathrm{Mg}$ (15\% de la surface) est écartée mais elle fera l'objet d'une analyse foliaire spécifique (voir section «Recourir à des échantillons foliaires spécifiques" page 46) pour déterminer la fertilisation corrective nécessaire et pour contrôler périodiquement la récupération des palmiers;

- lorsque l'unité de fertilisation présente plusieurs faciès dont les propriétés des sols ou la topographie peuvent induire des variations de rendement potentiel, il faut choisir celui qui contribue le plus au volume de régimes produit par l' unité de fertilisation. Certains faciès seront donc écartés mais des contrôles périodiques seront faits pour surveiller et s'il le faut, rectifier la nutrition minérale de ces zones (voir section "Recourir à des échantillons foliaires spécifiques" page 46).

Cette démarche est préférable à celle qui consiste à distinguer différents faciès (par exemple les bas-fonds et les plateaux) au sein d'une unité de fertilisation et de les représenter par des échantillons foliaires distincts. La principale raison est qu'il est difficile d'appliquer, sans erreur, des doses d'engrais différentes en fonction de la position de chaque palmier au sein des parcelles. 


\section{Encadré 6. Une fausse bonne idée : l'échantillon composite représentatif de l'ensemble de l'unité de fertilisation.}

Certains agronomes choisissent de prélever des palmiers selon une grille qui couvre toute la surface plantée de l'unité de fertilisation, en retenant par exemple 3 ou 4 palmiers par ligne, et 1 ligne sur 10. Ce système estime avec précision la composition moyenne des folioles de la population de l'unité de fertilisation, mais il ne rend pas compte des besoins spécifiques des différentes zones de l'unité de fertilisation et la décision «moyenne» qui sera prise à partir des teneurs de cet échantillon ne sera pas optimale.

Si l'unité de fertilisation est hétérogène (topographie, propriétés chimiques des sols, profondeur des sols et pratiques culturales), ce qui est souvent le cas, le potentiel de rendement et la disponibilité en éléments nutritifs varieront à l'intérieur de l'unité de fertilisation. En utilisant une teneur moyenne pour prendre une décision pour toute l'unité de fertilisation, on risque de recommander une dose d'engrais incorrecte pour une partie importante de la surface plantée.

Un exemple simplifié est illustré par la figure 22 et le tableau 4 : il s'agit d'une parcelle dont $85 \%$ de la surface sont couverts par des palmiers sains et $15 \%$ occupés par des palmiers déficients en magnésium. Une table de fertilisation très simple fait varier la recommandation en carbonate de magnésium $\left(\mathrm{MgCO}_{3}\right)$ en fonction de la teneur foliaire (tableau 4) : la recommandation correspondant à la teneur foliaire moyenne ne convient à aucune des deux classes de palmiers, elle est trop faible pour les individus déficients et inutile pour leurs homologues sains.

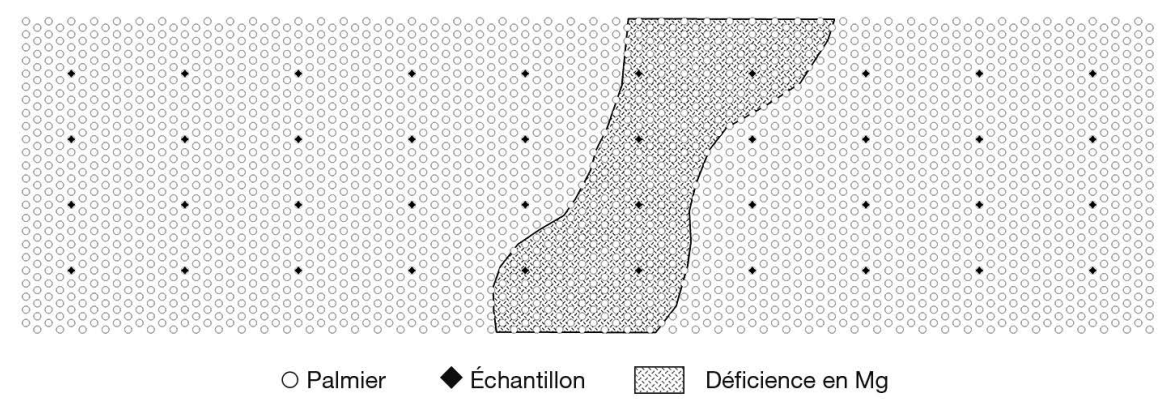

Figure 22. Échantillonnage systématique d'une unité de fertilisation hétérogène.

Une unité de fertilisation est occupée par des palmiers très déficients en Mg sur 15\% de sa surface (teneur des folioles en $\mathrm{Mg}: 0,08 \% \mathrm{MS}$ ) et des palmiers bien pourvus pour le reste de la surface $(\mathrm{Mg}: 0,25 \% \mathrm{MS})$. La teneur foliaire moyenne sera proche de $0,22 \%$ si l'échantillon représente proportionnellement les deux classes de palmiers.

Tableau 4. Table de fertilisation en $\mathrm{MgCO}_{3}$ ( $\mathrm{kg} /$ palmier/an).

\begin{tabular}{|c|c|c|c|c|c|}
\hline Teneur foliaire (\% MS) & & & 0,16 & 0,20 & 0,24 \\
\hline Dose de $\mathrm{MgCO}_{3}(\mathrm{~kg} /$ palmier/an) & 2,5 & 2,0 & 1,2 & 0,8 & 0,0 \\
\hline
\end{tabular}

On utilise cette table pour recommander une dose d'engrais en fonction de la teneur foliaire en $\mathrm{Mg}$.

Selon cette table, les palmiers déficients (Mg : 0,08\% MS) nécessitent 2,5 kg d'engrais alors que les palmiers sains (Mg: $0,25 \%$ MS) n'ont pas besoin d'être fertilisés. Or la teneur moyenne de $0,22 \%$ MS conduit à proposer une application de $0,8 \mathrm{~kg}$ d'engrais pour tous les palmiers, ce qui est totalement inadapté à la réalité. 


\title{
Illustration des choix d'unités entre faciès non majoritaires
}

Nous présentons l'exemple de trois unités de fertilisation contigües dans une plantation à topographie variée, où plusieurs dispositions sont possibles pour la localisation des palmiers de l'échantillon foliaire de référence (figure 23).

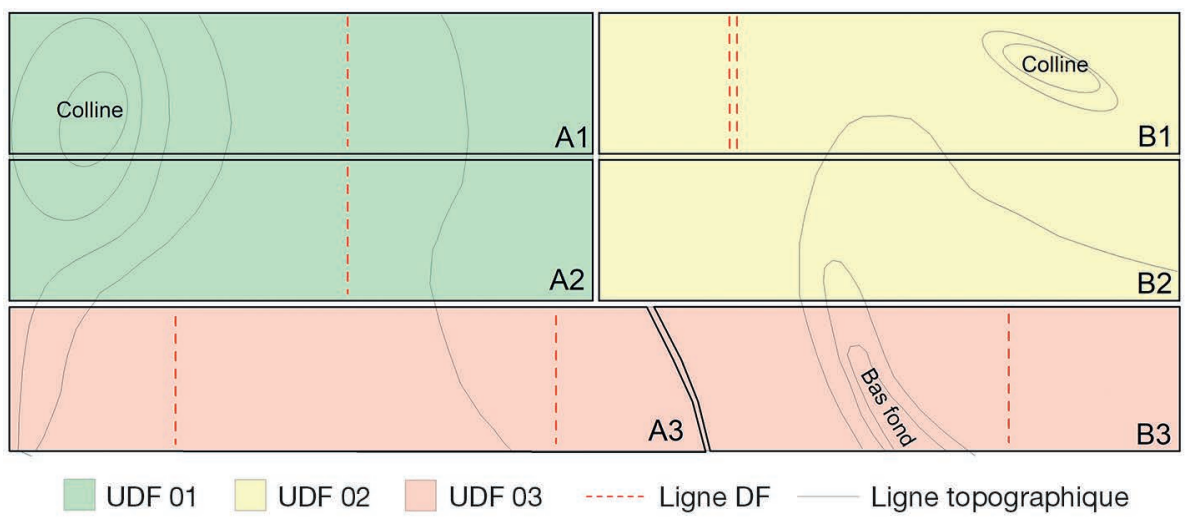

Figure 23. Exemples de choix des lignes pour prélever les échantillons foliaires.

\begin{abstract}
Trois unités de fertilisation (UDF 01, 02 et 03) ont été constituées en réunissant respectivement les parcelles $\mathrm{A} 1$ et $\mathrm{A} 2, \mathrm{~B} 1$ et $\mathrm{B} 2$ et $\mathrm{A} 3$ et $\mathrm{B} 3$. La topographie est composée de petites surfaces occupées par des collines et des petits cours d'eau dont les palmiers ne seront pas prélevés pour constituer les échantillons foliaires. Plusieurs choix sont possibles pour l'emplacement des lignes destinées au prélèvement des échantillons foliaires. Elles sont toujours situées dans les zones peu pentues qui couvrent la majeure partie des parcelles et sur lesquelles on a focalisé l'échantillonnage.

- pour l'UDF01, la position topographique intermédiaire (mi-pente) a été choisie et les deux lignes de prélèvement sont situées sur deux parcelles, mais en continuité pour faciliter le travail de collecte des folioles;

- pour l'UDF02, les deux lignes sont contigües, pour éviter à la fois le relief de colline et la tête de bas-fond; cette disposition en paires de lignes rend aisés le prélèvement et le contrôle du rang des feuilles prélevées;

- pour l'UDF03, plane et homogène, les palmiers prélevés sont répartis sur trois lignes qui couvrent l'ensemble de l'unité de fertilisation et évite le bas-fond.
\end{abstract}

\section{Quand, et sur quels critères, sélectionner les palmiers qui serviront aux prélèvements des échantillons?}

Il est impératif de pouvoir décider le plus tôt possible, c'est-à-dire dès 3 ans dans des conditions standard, de l'emplacement des palmiers qui permettront de prendre les décisions optimales de fertilisation. $\mathrm{Si}$ on dispose de cartes topographiques et pédologiques, les zones les plus propices seront présélectionnées. Pour le choix final, le stade jeune culture immature, permet d'effectuer des observations détaillées qui seront décisives, en particulier si l'on ne dispose pas d'autres sources d'information : - lors de la plantation, il est assez facile d'apprécier et de reporter sur plan les observations de terrain qui permettent de déceler les zones où la croissance des plants sera 
limitée. En examinant les trous de plantation, on peut déceler les problèmes d'hydromorphie, de variations de texture, de charges caillouteuses, d'horizons superficiels compactés. Cette opération donne en général une bonne appréciation de l'homogénéité des 30 premiers centimètres de sol. Elle peut être complétée par l'examen du sol à la tarière jusqu'à un mètre de profondeur;

- vers trois ans, avant de choisir où prélever pour le premier diagnostic foliaire de référence, la vigueur des plants peut être appréciée en mesurant par sondage la longueur des feuilles de rang 17. Les drones offrent aussi un excellent moyen d'observer et de vérifier l'homogénéité des parcelles afin de délimiter les zones où la croissance et les rendements seront limités (photo 8). Toutes les observations collectées (existence de zones gravillonnaires ou hydromorphes) seront reportées sur plan.

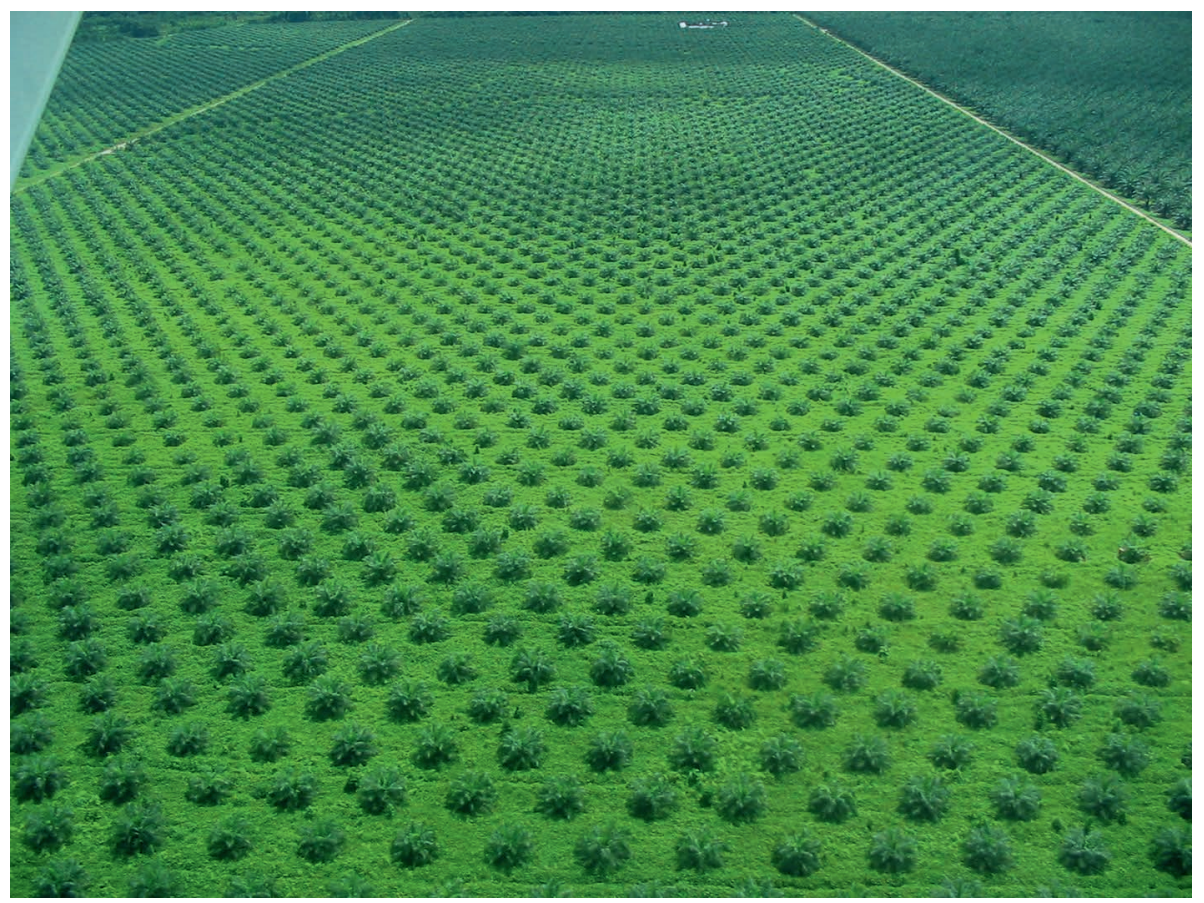

Photo 8. Vue aérienne d'une parcelle âgée de 2 ans.

Cette vue fait ressortir des hétérogénéités qui se traduisent par des différences de croissance, même si le relief est peu marqué. Les jeunes palmiers les moins développés souffrent probablement d'excès d'eau ce qui peut se répercuter sur la nutrition en azote. Cet âge est propice pour détecter les zones de forte mortalité et l'effet du matériel végétal si plusieurs croisements sont utilisés. Ces éléments sont pris en compte pour choisir les palmiers de l'échantillon foliaire de référence et également ceux des échantillons spéciaux (voir page suivante).

Une fois effectué le choix des lignes de prélèvement foliaire, il est indispensable de marquer à la peinture les palmiers pour s'assurer qu'on prélève toujours la même population. Cela permettra aussi de contrôler la qualité du prélèvement (respect du rang de la feuille, de la position des folioles). Le dispositif en paire de lignes (figure 23) 
est idéal pour observer l'état du feuillage (y-a-t-il des symptômes de déficience?) et les conditions du milieu qui peuvent expliquer les teneurs les plus faibles. Ces contrôles de terrain qui contribuent à la précision des décisions ne sont pas possibles lorsque l'échantillonnage couvre l'ensemble de l'unité de fertilisation (figure 22).

\section{Recourir à des échantillons foliaires spécifiques pour contrôler certaines zones de l'unité de fertilisation}

L'échantillon de référence doit être dédié à un seul faciès coïncidant avec une seule unité de sol ou une position topographique au détriment d'autres situations présentes dans le secteur. Cette méthode doit être adoptée en particulier lorsque des aménagements spéciaux sont entrepris sur de petites surfaces, par exemple pour lutter contre l'érosion (terrasses, bandes antiérosives).

Pour les zones non prises en compte dans l'échantillonnage foliaire de référence, il est recommandé de prélever des échantillons de contrôle périodiques, tous les 2 ou 3 ans, jusqu'à 5 ans, pour vérifier l'état de la nutrition minérale, qui est pilotée à partir du diagnostic de référence (figure 24).

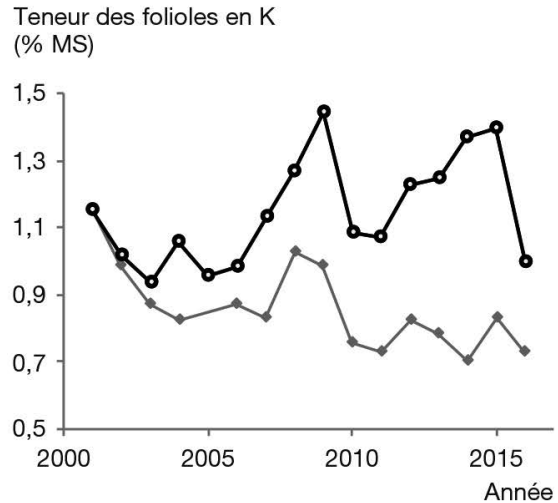

A $\rightarrow$ Spécial terrasses $\rightarrow$ DF principal

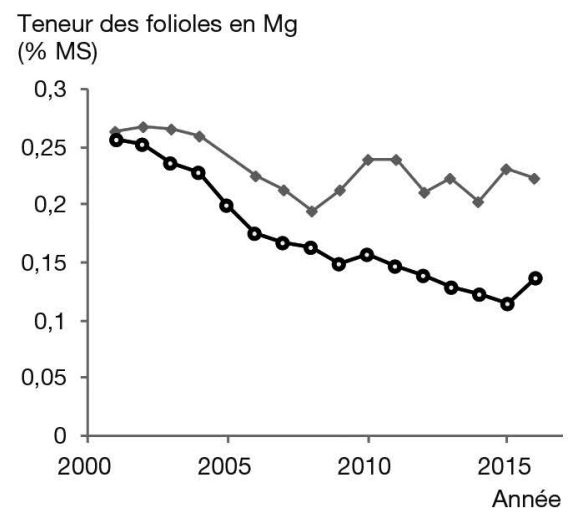

B $\multimap$ Spécial terrasses $\multimap-$ DF principal

Figure 24. Exemple d'échantillons foliaires spécifiques pour préciser la fertilisation en $\mathrm{K}$ (figure 24A) et en $\mathrm{Mg}$ (figure 24B) sur un faciès minoritaire (en terrasses) en Équateur.

Dans cette plantation des terrasses ont été créées au moyen d'engins, pour planter les palmiers situés sur les fortes pentes qui représentent $15 \%$ des surfaces. Ces terrasses reçoivent la même fertilisation que les palmiers des zones planes où sont placées les lignes de prélèvement des échantillons principaux. Quelques échantillons spéciaux ont été mis en place pour valider ou non les décisions prises pour les terrasses. Après plusieurs années, il apparaît que des épandages supplémentaires d'engrais potassique (par exemple $\mathrm{KCl}$ ) sont nécessaires sur les terrasses pour améliorer les teneurs en $\mathrm{K}$. Au contraire, les épandages d'engrais magnésien peuvent être diminués ou suspendus.

Des échantillonnages spécifiques sont également utiles lorsque des symptômes anormaux apparaissent, suggérant qu'une déficience s'installe dans certaines zones. Lorsque 
l'élément en cause n'est pas identifié avec certitude (c'est le cas pour les oligo-éléments, dont les déficiences sont rares), il faut prélever des échantillons sur des palmiers atteints de symptômes et aussi sur des palmiers témoins situés à proximité et ne présentant pas les symptômes. On compare ensuite les teneurs en éléments minéraux. Lorsqu'il est décidé d'appliquer localement un traitement correctif, les échantillons des palmiers déficients seront prélevés de nouveau pour évaluer l'amélioration des teneurs. 



\section{Adapter l'outil d'aide à la décision aux conditions locales : prendre en compte les spécificités de chaque site}

Nous disposons maintenant d'une méthode pour structurer une plantation en unité de fertilisation et obtenir chaque année des résultats d'analyse de bonne qualité.

Pour élaborer un diagnostic à partir de ces résultats analytiques, on les compare à des teneurs de référence que l'on affinera en fonction du contexte agronomique et environnemental de chaque situation. Les essais de fertilisation permettent de préciser les normes optimales pour les principaux éléments minéraux, et de mettre au point les tables de fertilisation à utiliser pour obtenir des teneurs satisfaisantes en tout lieu, lors de chaque contrôle annuel.

Lorsqu'on ne peut disposer de références locales pour suivre la nutrition minérale des palmiers, on utilise de façon arbitraire des teneurs parmi les normes communément admises par la profession (voir section "Connaître la variabilité des teneurs foliaires en éléments minéraux» page 22). On considère qu'il s'agit des teneurs foliaires optimales et on définit les tables de fertilisation qui permettent de les atteindre. Il faut cependant vérifier a posteriori que les teneurs qui ont été retenues comme optimales, le sont véritablement. La méthode la plus rigoureuse pour tester les valeurs initiales de pilotage de la fertilisation est la mise en place d'essais de fertilisation. Cette approche s'appuie sur les réponses des rendements et des teneurs foliaires aux apports d'engrais. Elle demande de 5 à 10 ans pour apporter ses conclusions mais elle permet de déterminer d'une part les déficiences natives qui limitent le rendement et d'autre part la plage de teneurs optimales au-dessus de laquelle le rendement n'est plus limité par l'élément nutritif étudié. Les essais renseignent aussi sur les teneurs qu'il est possible d'atteindre avec les doses d'engrais testées dans les conditions de l'essai. 


\section{Principes des essais de fertilisation}

\section{Choix des traitements et des dispositifs}

Il s'agit de tester l'influence des principaux éléments minéraux sur le rendement dans les conditions de sol et de climat de la plantation. Les éléments à tester sont retenus en fonction des informations préalables dont on dispose (par exemple des analyses de sol qui indiqueraient que les réserves en $\mathrm{P}, \mathrm{K}$ et $\mathrm{Mg}$ sont faibles). Le potassium, élément le plus consommé par le palmier à huile, est presque toujours apporté sous forme de chlorure de potassium $(\mathrm{KCl})$. De ce fait, on teste aussi l'influence du chlore : il faut en tenir compte lors de l'interprétation des résultats puisque la déficience en chlore existe (Ollagnier et Ochs, 1971).

Les essais sont généralement des dispositifs factoriels qui combinent plusieurs éléments apportés chacun sous forme d'engrais à différentes doses et qui testent toutes les combinaisons possibles. Les dispositifs factoriels sont intéressants car ils permettent de comparer entre elles les doses d'un engrais donné en ayant plusieurs répétitions de chaque comparaison mais sans avoir à répéter l'ensemble du dispositif ce qui aurait l'inconvénient de couvrir de grandes surfaces cultivées. Ils permettent en outre plusieurs types d'analyses, des plus simples aux plus compliquées, selon les résultats que l'on attend de l'essai.

On recommande de tester de vrais témoins sans apport de l'élément à étudier (N0, $\mathrm{P} 0, \mathrm{~K} 0 \ldots . .$.$) . Ces témoins renseignent à la fois sur les seuils de teneurs déficientes et$ sur la résilience des réserves dans le sol, comme on le verra plus loin (section «Évaluer les réserves du sol» page 74).

Les autres doses sont choisies en progression arithmétique (dose a, dose 2a, dose $3 \mathrm{a} . .$. ). L'étendue des doses choisies varie avec l'âge des palmiers et couvre une gamme de besoins définie en fonction du type de sol, du climat et de la production potentielle. En particulier avant cinq ans, les doses appliquées ne représentent qu'une fraction $\mathrm{F}$ de ce qui sera appliqué plus tard mais il importe de conserver la progression arithmétique (dose $1 \mathrm{Fa}$, dose $2 \mathrm{Fa}$, dose $3 \mathrm{Fa} .$. avec $\mathrm{F}<1$ ). Comme on cherche à déterminer des seuils de déficience (sans apport, dose « 0 ») et les plages de teneurs optimales, la première dose non nulle (dose a) doit être proche de la dose ciblée pour atteindre le rendement maximum. Lorsqu'on n'observe pas, ou très peu, d'amélioration de la productivité en doublant cette dose (dose $2 \mathrm{a}$ ), on obtient un résultat très convaincant pour justifier d'une fertilisation raisonnée (encadré 7).

Le matériel végétal peut aussi faire partie des facteurs étudiés lorsque des origines différentes ont été plantées. On peut mettre en place des essais pour chaque origine dont on estime que la surface plantée est suffisamment vaste pour justifier davantage de précision. Lorsqu’on s'interroge sur des besoins spécifiques de différents matériels végétaux, il faut un dispositif avec un facteur génétique (étude nutrition minérale $\mathrm{x}$ matériel végétal). Ces dispositifs sont rares, ils intéressent principalement la production de semences et le matériel hybride $\mathrm{O} \times \mathrm{G}$ dont certains croisements ont des comportements spécifiques. D'autres types de dispositifs existent pour d'autres usages, comme les «plans de mélange" pour tester des produits et des formulations comprenant plusieurs éléments, 
ou les "plans centrés composites» pour estimer des surfaces de réponse. Ces plans peuvent être très économiques en surface cultivée (certaines combinaisons ne sont pas répétées) mais leur analyse peut être complexe et ils sont sensibles au manque de données sur certaines parcelles et aux erreurs d'appréciation sur le caractère négligeable de certaines interactions. Cela les rend mal adaptés aux essais de longue durée qui ne peuvent pas être réitérés en cas d'échec.

\section{Encadré 7. Un dispositif factoriel très efficace pour le palmier à huile :}

\section{3 facteurs à 3 niveaux.}

Le nombre souvent important de combinaisons à tester dans un dispositif factoriel et la taille minimale que l'on doit donner aux parcelles élémentaires font qu'on ne peut pas garantir que la surface de l'essai sera parfaitement homogène ce qui peut perturber les résultats. On préférera donc des dispositifs permettant de contrôler les variations de fertilité du terrain en constituant des blocs incomplets confondus avec des interactions de rang élevé que l'on sait pouvoir négliger. C'est le cas du dispositif factoriel combinant 3 facteurs à 3 niveaux $\left(3^{3}\right)$ qui permet une répartition des 27 combinaisons (figure 25) en 3 blocs confondus avec l'interaction des trois facteurs qui a rarement des effets agronomiques identifiables. Ce dispositif peut être planté sans répétition des 27 combinaisons, l'estimation correcte de l'erreur résiduelle étant possible car les interactions entre deux facteurs sont en général simplement linéaires. Ce dispositif est efficace en raison de la qualité des réponses observées par rapport à la surface occupée (Yates, 1964 p. 42 et 53).

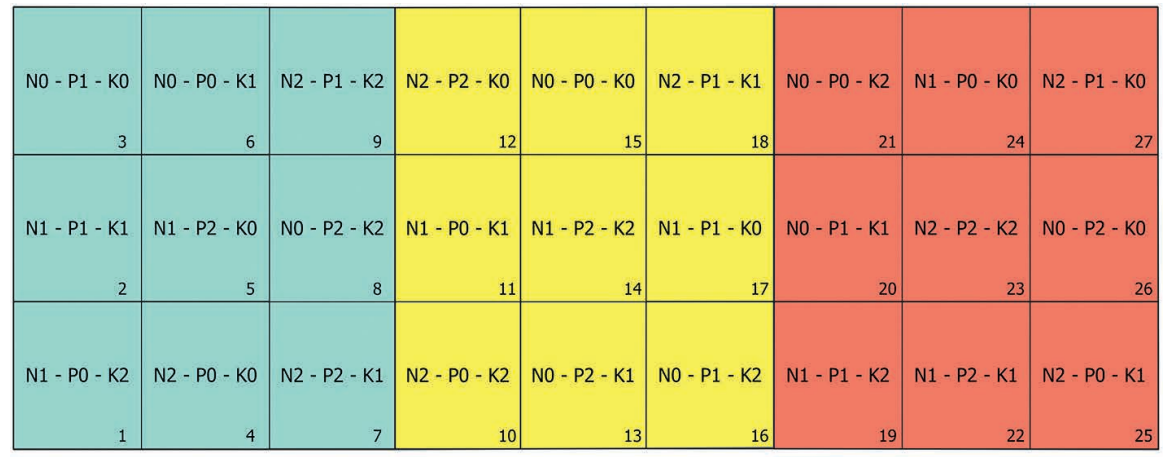

$\square$ Bloc $1 \square$ Bloc $2 \square$ Bloc 3

Figure 25. Dispositif factoriel de 3 facteurs à 3 niveaux comprenant 27 parcelles élémentaires.

Le numéro de chaque parcelle élémentaire apparaît en bas à droite et la combinaison des 3 facteurs est affichée au centre de chaque rectangle. Les parcelles 1 à 9,10 à 18 et 19 à 27 ont été regroupées en trois blocs incomplets dans lesquels les traitements ont été choisis pour permettre une analyse correcte des résultats. Dans chaque bloc apparaissent 3 combinaisons avec N0, 3 combinaisons avec N1 et 3 combinaisons avec N2. Il en va de même pour les facteurs P et K. Cette particularité permet lors de l'analyse de la variance, de calculer un effet bloc qui diminuera d'autant la variance résiduelle.

\section{Mettre en place un essai homogène}

Lobtention de réponses dans le cadre d'un essai, demandera plusieurs années de suivi; elles sont souvent dépendantes du type de sol et parfois du matériel végétal. Il faut donc réfléchir aux priorités pour la plantation : quel est le type de sol le plus représenté? 
Quel matériel sera planté dans le futur? Les essais sont généralement contemporains des programmes de plantation; pour un pilotage efficace de la fertilisation, ils doivent fournir le plus rapidement possible des informations précises.

L'objectif d'un essai de fertilisation est souvent de déterminer les teneurs optimales pour la période la plus productive des plantations, c'est-à-dire entre 8 et 15 ans. Cependant, les essais ne sont généralement pas poursuivis après 10 ans. On a tout intérêt à débuter l'application des traitements le plus tôt possible pour éviter que les réserves présentes dans les biomasses des palmiers et dans le sol ne retardent l'apparition d'une déficience. Un essai démarre généralement à 3 ans mais pour certains éléments comme $\mathrm{K}$ et $\mathrm{Cl}$, il est possible de commencer dès l'année de plantation. Les doses appliquées dans les premières années de l'essai augmentent en même temps que la biomasse mais il faut atteindre rapidement les doses définitives. Un protocole simple facilite toujours la synthèse des résultats en fin d'essai.

Les parcelles expérimentales d'un essai sont des groupes compacts de palmiers auxquels sont appliquées les différentes fertilisations, par exemple les 27 combinaisons d'un essai factoriel $\left(3^{3}\right)$. Les observations élémentaires (production, analyses foliaires...) proviennent des palmiers utiles situés au centre des parcelles expérimentales et entourés de palmiers de bordure recevant la même fertilisation (figure 26). La taille des parcelles expérimentales est un point clef de la conception de l'essai. D'une part pour des raisons pratiques (volume de travail, risques d'erreur, difficulté à disposer de conditions de sol homogènes sur une surface étendue), on cherche à mettre en place des essais à faible surface. D'autre part il faut un nombre minimal de palmiers pour observer correctement l'effet d'une combinaison de traitements sur le rendement. Ainsi, un effectif de 9 palmiers utiles par parcelle élémentaire est un bon compromis dans une situation standard. Cet effectif peut être plus élevé du fait que certains individus peuvent disparaitre avant la fin de l'essai; la surface d'un essai est variable selon le contexte sanitaire. Par exemple, un essai factoriel à 3 facteurs et à 3 niveaux $\left(3^{3}\right)$ ayant des parcelles de 5 lignes de 5 palmiers (dont 9 palmiers utiles) couvre 4,7 ha (143 palmiers/ha). Un essai factoriel $\left(3^{3}\right)$ ayant des parcelles de 6 lignes de 7 palmiers (20 palmiers utiles) couvre 7,9 ha.

L'homogénéité des essais est cruciale pour obtenir des résultats de qualité et des réponses précises. Pour garantir cette homogénéité, des choix sont faits à chaque étape de préparation de l'essai :

- production des plants et sélection en fin de pépinière, pour ne planter que des palmiers "normaux» et homogènes;

- choix de la parcelle : un seul type de sol et si possible un seul type de précédent cultural, sur une parcelle plane ou à défaut faiblement pentue;

- contrôle des ravageurs, pour éviter les pertes de plants et les retards de croissance les premières années. Il y a des effets prévisibles de bordure à éviter, comme la mineuse des racines du palmier à huile (Sagalassa valida) présente en lisière de forêt, ou les rongeurs en bordure de bas-fond.

Si l'essai est mis en place dans une culture existante, un indicateur de croissance végétative, comme la longueur des feuilles, peut servir à détecter les gradients de fertilité présents dès le début de l'essai et trouver la meilleure disposition pour l'essai (figure 27). 


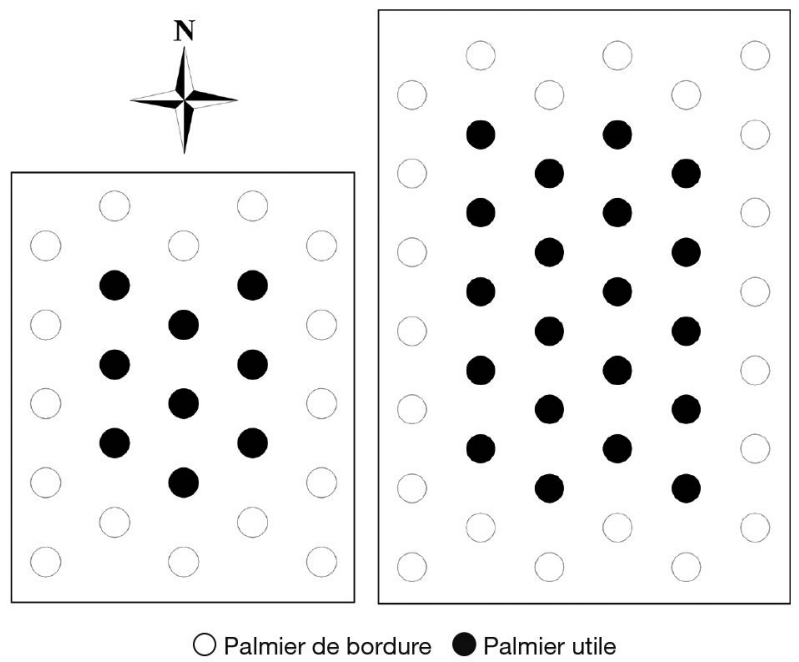

Figure 26. Deux exemples de parcelles expérimentales de tailles différentes.

Parcelle $1: 25$ palmiers; parcelle $2: 42$ palmiers.

Tous les palmiers des parcelles expérimentales sont fertilisés selon une modalité prévue par le protocole. Les palmiers de bordure limitent l'influence des modalités d'une parcelle à l'autre, sur les palmiers utiles qui servent aux observations. La taille des parcelles dépend du contexte sanitaire, de la surface homogène disponible pour l'essai mais aussi d'autres critères comme l'organisation des pesées individuelles des régimes récoltés. Tous les palmiers doivent porter une étiquette permettant de les identifier sans erreur, les limites des parcelles expérimentales sont matérialisées à chaque coin par des pancartes.

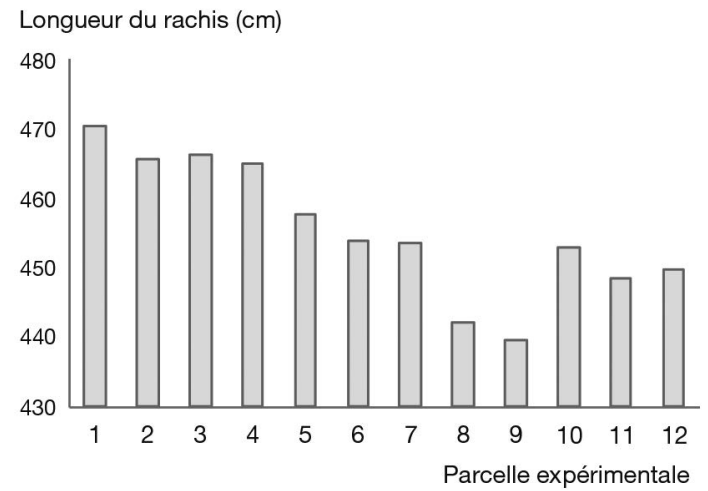

Figure 27. Longueur moyenne de la feuille de rang 17 à 5 ans, par parcelle expérimentale avant le début du protocole.

L'essai prévoit de tester l'application de bore à deux niveaux (présence-absence) avec 6 répétitions. Les parcelles expérimentales sont situées le long de l'axe principal de la parcelle qui coïncide avec un gradient de développement végétatif. En conséquence, les répétitions doivent être placées le long de cet axe. 
Malgré ces précautions, les variations des propriétés des sols sont souvent difficiles à déceler. C'est pourquoi il est important de favoriser l'homogénéité à l'intérieur des blocs (complets ou incomplets). Si un gradient est suspecté (pente, grand axe de la parcelle), les blocs sont placés le long de ce gradient. Le dispositif factoriel $\left(3^{3}\right)$ peut être réparti en 3 blocs, ce qui permet de respecter cette précaution (figure 28).

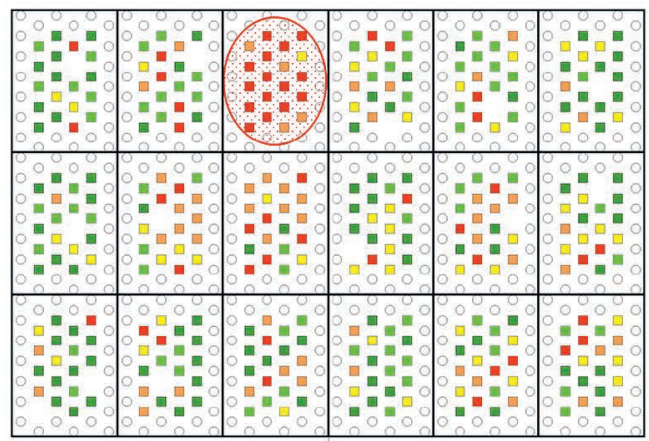

Bloc $1-221 \mathrm{~kg} /$ palmier/an

Production de régimes ( $\mathrm{kg} /$ palmier/an)

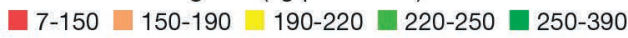

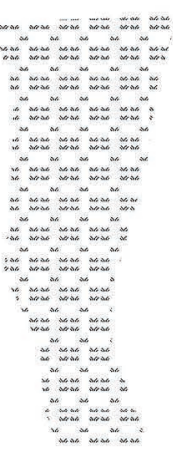

Bloc $2-229 \mathrm{~kg} /$ palmier/an

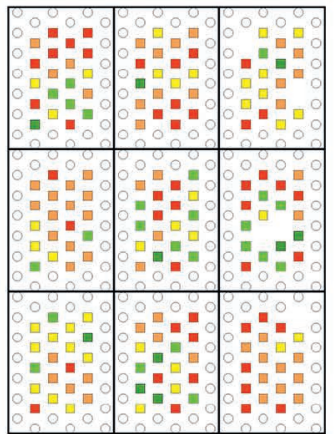

Bloc $3-185 \mathrm{~kg} /$ palmier/an

Zone humide Déficience en $\mathrm{K}$

Figure 28. Exemple d'un dispositif factoriel $\left(3^{3}\right)$ dans lequel les parcelles expérimentales ont été regroupées en 3 blocs.

\begin{abstract}
Les productions moyennes de 9 à 11 ans font ressortir un effet bloc significatif qui se traduit par une production moyenne du bloc $3(185 \mathrm{~kg} /$ palmier $)$ inférieure à celles des deux autres blocs (bloc 1, 221 kg/palmier; bloc 2, $229 \mathrm{~kg} /$ palmier) : la classe de productivité individuelle la plus faible est en rouge. La parcelle est plane et une zone hydromorphe est évitée entre les blocs 2 et 3 mais, malgré cette précaution, la variabilité entre blocs ne pouvait être détectée avant la mise en place de l'essai. Une autre singularité est aussi apparue en observant les teneurs foliaires en $\mathrm{K}$ : dans une parcelle expérimentale $\mathrm{du}$ bloc 1, la baisse des teneurs en $\mathrm{K}$ et la plus faible production des palmiers s'expliquent par des réserves en $\mathrm{K}$ du sol plus faibles que dans le reste de l'essai.
\end{abstract}

Lors de la mise en place d'un essai, il ne faudra pas négliger l'étiquetage des arbres et l'identification des traitements à appliquer sur chaque parcelle expérimentale. Il conviendra d'organiser précisément la collecte et l'enregistrement des données pour assurer leur conservation sur toutes les années où durera l'essai.

\title{
Agréger les données et déterminer les teneurs optimales locales
}

Pour chaque combinaison des facteurs étudiés ou chaque traitement appliqué sur une parcelle expérimentale, on utilise les palmiers utiles pour déterminer par analyse les teneurs d'un échantillon foliaire composite, pour calculer la production moyenne sur une période donnée à partir des pesées individuelles des régimes, et pour calculer les valeurs moyennes des observations végétatives (longueurs et émission de feuilles). Même si on a pris des précautions en sélectionnant rigoureusement les plants de la pépinière, des palmiers qui sortent des standards peuvent apparaitre après quelques 
années, généralement à l'entrée en production. Il s’agit soit d'anormalités (rachitisme, plants abortifs) soit de maladies et ces individus ne sont pas pris en considération pour le calcul de l'effet moyen sur les variables observées dans chaque parcelle expérimentale. Il faut cependant les identifier selon des normes strictes. Les déficiences qui se mettent en place dans les essais ont un impact sur l'aspect végétatif des palmiers qu'il ne faudrait pas confondre avec une anomalie génétique. Les analyses statistiques sont généralement faites sur les valeurs parcellaires annuelles (teneur des échantillons composites ou production moyenne par palmier). Les moyennes obtenues pour les modalités de chaque facteur permettent de suivre l'évolution dans le temps de la nutrition minérale et de comprendre la mise en place des déficiences qui limitent les rendements (figure 29).

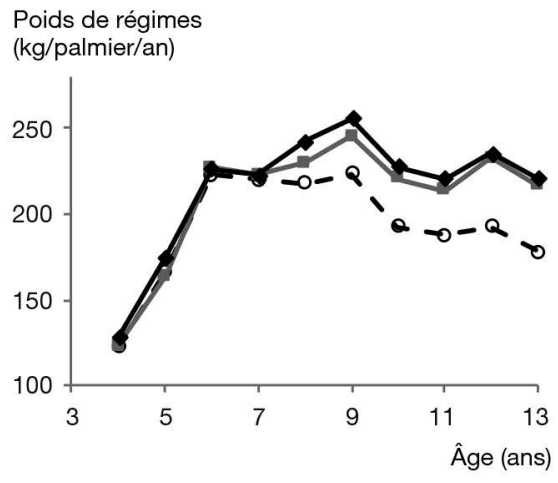

$\mathrm{A} \quad-\mathrm{G} 0 \longrightarrow \mathrm{K} 1 \longrightarrow \mathrm{K} 2$

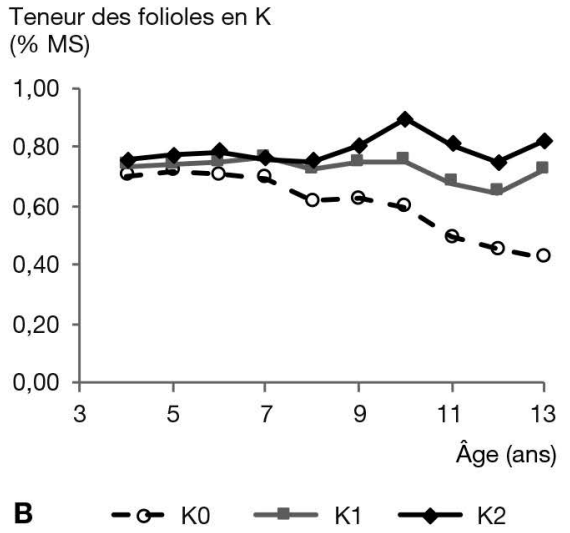

À partir de 6 ans, les doses de $\mathrm{KCl}$ sont : K0, témoin; K1, $1 \mathrm{~kg} /$ palmier/an et $\mathrm{K} 2,3 \mathrm{~kg} /$ palmier/an.

Figure 29. Évolution de la production annuelle de régimes (figure 29A) et des teneurs foliaires en $\mathrm{K}$ (figure 29B) dans un essai factoriel à 3 facteurs de fertilisation (N P K) et à 3 niveaux, en Équateur.

\section{Précision expérimentale}

Pour chaque essai de fertilisation, il est possible de calculer a posteriori, la différence minimale qu'il est possible de détecter (MDD, minimum detectable difference) avec une probabilité raisonnable. On fixe généralement cette probabilité (appelée aussi puissance) à $75 \%$. Ce calcul est intéressant lorsqu'il n'y a pas d'effet significatif des traitements sur le rendement bien qu'on ait appliqué les traitements pendant plusieurs années. Plutôt que de conclure que les facteurs n'ont pas d'effet sur les rendements, on peut conclure que les écarts entre deux modalités sont probablement inférieurs à la MDD. Si celle-ci est faible, recommander une modalité plutôt qu'une autre aura peu d'impact. Dans le cas contraire, il est prudent de tester de nouveau les facteurs dans un dispositif plus adapté (parcelle de plantation homogène, plus grand nombre d'arbres, faible mortalité etc.) 
Du fait de la grande variation de la MDD d'un essai à l'autre, il vaut mieux l'exprimer en pourcentage du rendement moyen de l'essai. Par exemple, au cours de 15 essais de longue durée, menés dans différents pays et conduits jusqu'à 21 ans pour les plus longs, la MDD a été calculée pour une puissance de $75 \%$ : elle a varié de 7 à $30 \mathrm{~kg}$ de régimes par palmier et par an car les rendements potentiels étaient très variables selon les situations agroécologiques. Cette enquête a montré aussi que la MDD est rarement inférieure à $5 \%$ du rendement (1 essai sur 15 ) alors qu'il est fréquent d'observer des MDD comprises entre 5 et $10 \%$ du rendement ( 9 essais sur 15), ce qui constitue semble-t-il la norme pour considérer qu'un essai a été mené correctement. Au contraire les résultats des 5 essais de notre enquête dont les MDD étaient supérieures à $10 \%$ n'apportent pas d'information précise sur l'impact des facteurs étudiés sur le rendement. Il serait souhaitable de mettre en place de nouvelles expériences dans des conditions mieux contrôlées.

\section{Construire la table de fertilisation à partir des résultats expérimentaux}

Cette étape est fondamentale, puisque l'on cherche à transformer des résultats statistiques (le plus souvent une ANOVA) en une prise de décision pour la fertilisation. Il faut d'abord cerner quelles sont, élément par élément, les plages de teneurs optimales, selon les résultats de l'essai. Ces teneurs optimales sont celles, au-delà desquelles le gain de rendement dû aux engrais est jugé trop faible pour être intéressant. On construira ensuite les tables de fertilisation qui permettront d'atteindre ces objectifs dans chaque secteur de fertilisation.

\section{Déterminer la plage des teneurs optimales pour un élément}

Deux approches sont possibles selon le traitement des résultats de l'essai.

\section{Méthode par l'observation de seuils de rupture}

Pour appliquer cette méthode, on recherche une période de l'essai où l'on observe des différences de rendement significatives entre modalités et on examine les teneurs obtenues avec ces modalités. En particulier, on s'intéresse aux changements suivants : - lorsqu'une modalité étudiée, par exemple K0, ne permet plus d'atteindre le rendement que l'on peut obtenir avec une autre modalité (K1 ou K2) et que l'écart devient significatif, la teneur en $\mathrm{K}$ associée à $\mathrm{K} 0$, caractérise un état de déficience. La plage de teneurs optimales en potassium doit être supérieure à la teneur déficiente; - lorsque l'écart de rendement entre deux modalités fertilisées, par exemple entre K1 et K2, n'est pas significatif, on considère qu'avec la première modalité, on est proche d'un état nutritionnel satisfaisant qui peut servir de référence.

L'observation de ces changements est issue de la dynamique des résultats de l'essai. On identifie à partir de quelle teneur une perte de rendement est devenue mesurable statistiquement. Il y a un décalage d'un an au minimum, plus généralement deux ans, entre la variation de la teneur foliaire et son effet sur le rendement. 
Pour illustrer cette démarche, nous avons repris l'exemple de la figure 29. Le passage de la teneur foliaire en $\mathrm{K}$ de 0,70 à $0,60 \% \mathrm{MS}$, entre 7 et 8 ans, serait à l'origine de la différence de rendement entre $\mathrm{K} 1$ et K0, qui est significative à partir de 9 ans : le seuil de déficience en $\mathrm{K}$ serait donc compris entre ces deux valeurs. On peut donc borner la plage des teneurs optimales au minimum à $0,65 \% \mathrm{MS}$ - voire $0,70 \% \mathrm{MS}$ par prudence. On observe aussi dans cet essai que pour $\mathrm{K} 1(1 \mathrm{~kg} \mathrm{KCl} / \mathrm{palmier} / \mathrm{an})$ les teneurs foliaires sont stables sur la durée de l'essai avec une valeur moyenne de 0,73\% MS. Il ne sert alors à rien de dépasser cette teneur puisque les rendements mesurés pour K1 et K2 ne sont pas différents. Cette teneur se situe donc dans la zone des teneurs optimales, que l'on peut fixer à 0,70-0,75\% MS, et qui servira à construire la table de fertilisation.

Les essais renseignent aussi sur les doses d'engrais utiles pour atteindre notre objectif. Dans notre exemple (figure 29), la dose $\mathrm{K} 1 \mathrm{de} 1 \mathrm{~kg} \mathrm{KCl} / \mathrm{palmier} / \mathrm{an}$ peut être proposée pour maintenir les teneurs foliaires entre 0,70 et $0,75 \%$ MS. La dose K2 ( $3 \mathrm{~kg} \mathrm{KCl} / \mathrm{palmier} / \mathrm{an})$ ne doit être proposée que pour corriger des teneurs très faibles : elle n'est pas applicable sur de longues périodes pour des raisons économiques.

La méthode proposée dépend fortement de la possibilité d'observer une ou plusieurs différences de rendement significatives entre les modalités et la qualité des données de l'essai est primordiale pour traduire cela en seuils de teneurs.

Les résultats expérimentaux ne sont pas toujours significatifs et d'autres règles de décision peuvent être utilisées, même si elles restent subjectives. Une augmentation de production inférieure à $5 \%$ entre deux modalités, par exemple entre K1 et K2, n'est en général pas significative. Inversement, si une modalité induit un gain de production de $10 \%$ ou plus, ce gain est le plus souvent significatif; si ce n'est pas le cas, il faut examiner la qualité des données de l'essai.

Foster (2003) se réfère à une variation du rendement en tonnes régimes/ha/an : en dessous d'une variation de 0,5 tonnes/ha/an, il n'y a pas lieu de modifier une modalité pour une autre; pour une variation comprise entre 0,5 et 2 tonnes/ha/an, un ajustement est à rechercher; au-delà d'une baisse de 2 tonnes/ha/an, il est impératif de corriger la déficience qui limite le rendement.

Quelle que soit la méthode que l'on adopte pour transformer des résultats expérimentaux en barème de fertilisation, il faut examiner la précision des résultats et calculer la MDD pour tirer des conclusions correctes (voir section précédente «Précision expérimentale»). En effet, s'il n'y a pas d'effets significatifs entre les modalités après plusieurs années d'observation, il serait hasardeux de conclure que les facteurs testés n'ont pas d'effet sur le rendement. Des différences de rendement entre modalités peuvent être élevées sans pour autant être significatives en raison de résultats peu précis.

\section{Méthode par la modélisation des résultats expérimentaux}

La plage des teneurs optimales peut aussi être déterminée par transformation mathématique des résultats moyens de l'essai. Pour cela, il faut modéliser les réponses du rendement et des teneurs foliaires aux doses d'engrais et calculer précisément les teneurs qui correspondent à un seuil de rentabilité économique de l'engrais que l'on a fixé. 
La première étape consiste à identifier une période où les effets sont considérés comme exprimés (figure 29 : période de 10-13 ans). Cette période représente un rendement moyen correct et atteignable sur l'ensemble de la plantation en culture mature à partir de 10 ans.

La deuxième étape consiste à calculer les équations des courbes de réponse des teneurs foliaires et des rendements aux doses d'engrais de l'essai en adoptant par exemple un modèle de Mitscherlich :

$$
\mathrm{Y}=\mathrm{a}-\mathrm{b} \exp (\mathrm{cX})
$$

$\mathrm{Y}$ est la variable à expliquer (rendement, teneur foliaire) ; $\mathrm{X}$ est la variable explicative (dose d'engrais); a, b et c sont des constantes.

À partir de la courbe de réponse du rendement, on détermine une dose d'engrais économiquement optimale (DEO) compte tenu des paramètres économiques moyens. Cette approche d'équilibre optimal a été décrite par Caliman et al. (1994, figure 30). On détermine aussi la teneur foliaire cible, ou "niveau critique», et la dose d'engrais pour atteindre cette teneur. La table de fertilisation sera construite à partir de ces valeurs.
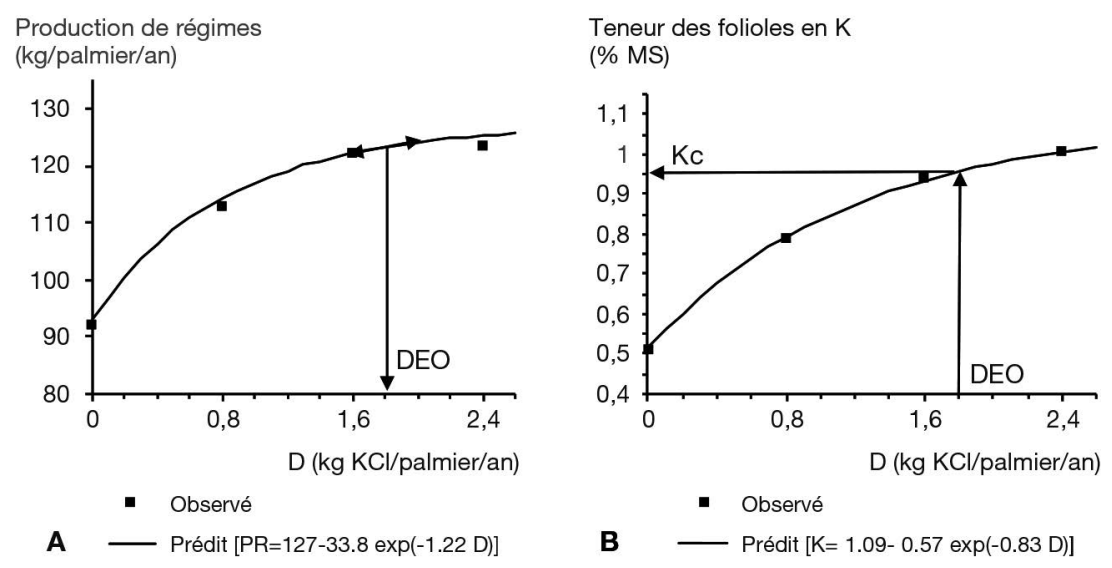

Figure 30. Exemple de courbes de réponse de la production (Figure 30A)

et des teneurs foliaires (Figure 30B) aux doses d'engrais (D) (d'après Caliman et al., 1994).
Les équations ont été ajustées selon le modèle de Mitscherlich. En tout point de la courbe A la valeur de la tangente indique le rapport $\mathrm{kg}$ régimes $/ \mathrm{kg}$ engrais. Il diminue avec les apports de $\mathrm{KCl}$ jusqu'à atteindre une valeur qui reste économiquement acceptable. On détermine la dose d'engrais économiquement optimale (DEO) correspondante; elle est de $1,7 \mathrm{~kg} \mathrm{KCl} / \mathrm{palmier} / \mathrm{an}$. La figure $30 \mathrm{~B}$ permet de déterminer la teneur foliaire en potassium qui est obtenue avec cette dose d'engrais. Cette valeur, $\mathrm{Kc}=0,95 \% \mathrm{MS}$, servira de référence pour la gestion de la nutrition potassique dans plantation où a été mis en place l'essai.

Cette approche peut être généralisée à des surfaces de réponse en combinant les facteurs deux à deux. Le traitement mathématique des surfaces de réponse permet de calculer les doses de chaque élément et les teneurs foliaires correspondantes en fonction des paramètres économiques retenus par l'utilisateur. Un exemple complet de traitement d'un essai a été fourni par Webb (2009). 
Ces méthodes de calcul sont basées sur des critères rigoureux mais demandent une projection réaliste des coûts des intrants et du marché de l'huile de palme sur de longues périodes, puisque le palmier répond aux engrais avec un délai d'un à deux ans. La simulation faite par Webb (2009) montre que le cours de l'huile et le coût des engrais influent fortement le rendement escompté et des doses d'engrais qui permettent de l'atteindre. Un bon paramétrage est donc nécessaire.

\section{Construire une table de fertilisation à partir de la plage des teneurs optimales}

Une fois déterminée une valeur optimale pour un élément donné, on définira une plage de teneurs optimales autour de cette valeur et on construira un barème de fertilisation. Il est constitué de classes de teneurs qui préconisent des doses spécifiques d'engrais. La classe la plus importante est celle qui contient la teneur optimale, $\mathrm{Kc}=0,95 \% \mathrm{MS}$ dans le cas de la figure 30. Pour maintenir les teneurs en potassium dans cette classe, on appliquera la dose d'engrais économiquement optimale $(\mathrm{DEO}=1,7 \mathrm{~kg} \mathrm{KCl}) . \mathrm{Si}$ la teneur foliaire de l'échantillon est située dans une classe inférieure, on préconisera une dose d'engrais supérieure à la DEO et réciproquement (tableau 5).

Tableau 5. Barème de fertilisation en $\mathrm{KCl}(\mathrm{kg} / \mathrm{palmier} / \mathrm{an})$ en fonction de la teneur des échantillons foliaires.

\begin{tabular}{lccccc}
\hline Teneurs en $\mathrm{K}(\% \mathrm{MS})$ & $<0,8$ & 0,8 à 0,9 & 0,9 à 1,0 & 1,0 à 1,1 & $>1,1$ \\
\hline Dose de $\mathrm{KCl}(\mathrm{kg})$ & 2,7 & 2.2 & 1,7 & 1 & 0 \\
\hline
\end{tabular}

La table de fertilisation est centrée sur la plage des teneurs optimales et la dose d'engrais économiquement optimale qui ont été déterminées à partir des résultats de l'essai. Lorsque les teneurs sont situées dans des classes élevées on diminue la recommandation de fertilisation jusqu'à ne plus appliquer d'engrais pendant quelques années, si nécessaire. Au contraire, les plages des teneurs les plus faibles recommandent des doses plus élevées. On a choisi, ici, de plafonner la dose maximale à $2,7 \mathrm{~kg} \mathrm{KCl} /$ palmier/an.

Cependant, Webb (2009) a précisé que l'utilisation d'une table de fertilisation ne convient qu'aux parcelles dont les conditions de matériel végétal et de sol sont proches de celles de l'essai. Cette remarque est fondamentale car elle a des conséquences sur le besoin en données expérimentales et sur la construction des barèmes de fertilisation :

- s'il y a plusieurs unités de sol dont les propriétés physiques et chimiques sont suffisamment différentes pour influencer la nutrition minérale, il faut mettre en place des essais sur les unités les plus grandes. Cette précaution est importante puisque sur certains sols, la réponse des teneurs foliaires en $\mathrm{K}$ aux applications de $\mathrm{KCl}$ peut être faussée par l'abondance du calcium dans le sol, comme on l'examinera plus loin; - lorsque le réseau expérimental est jeune, avec peu de teneurs de référence pour le pilotage de la fertilisation, on n'a pas d'autre choix que d'extrapoler les résultats à l'ensemble de la plantation. En conséquence, il faudra analyser spatialement les teneurs foliaires de la plantation pour détecter celles dont la réponse aux engrais 
n'est pas conforme aux résultats expérimentaux. On peut ainsi modérer les apports d'engrais si on soupçonne l'existence d'un problème d'absorption ou de translocation des éléments vers les folioles (encadré 8).

\section{Encadré 8. La rentabilité des apports d'engrais et la préservation du milieu.}

Il n'est pas rare de constater que les rendements mesurés dans un essai continuent d'augmenter lorsqu'on passe d'une modalité à une autre même lorsque l'écart n'est pas significatif. On pourrait donc être tenté d'atteindre les teneurs foliaires les plus élevées de l'essai et d'appliquer les doses plus élevées d'engrais, mais il faut se garder de viser le rendement maximal obtenu expérimentalement et se préoccuper du devenir de l'engrais pour les doses les plus fortes.

Si les éléments minéraux ne sont pas ou peu absorbés par la culture, que deviennent-ils? S'ils s'accumulent dans les parties végétatives de la plante ils retourneront au sol par le biais de l'élagage ou au moment de la replantation lors de l'abattage des troncs. Il est à craindre qu'à un moment ou à un autre, ils soient en excès par rapport à la capacité de stockage du sol. C'est pourquoi par prudence vis-à-vis du milieu environnant il faut prévoir dans la table de fertilisation qu'à partir d'une certaine teneur foliaire les apports d'engrais ne sont plus nécessaires.

\section{Exploiter les conclusions de l'approche expérimentale}

Les essais de fertilisation permettent de détecter rapidement les éléments minéraux clef qui limitent les rendements dans un contexte donné. Ils renseignent sur les seuils de déficience, qui serviront à fixer les teneurs minimales à atteindre sur toutes les unités de fertilisation. Ils précisent aussi à partir de quel seuil les doses d'engrais augmentent les teneurs foliaires sans améliorer le rendement.

Une modélisation plus poussée des résultats expérimentaux est possible via les courbes ou les surfaces de réponse, qui permettent d'obtenir des normes précises, valables dans les contextes des parcelles d'essai; on considère que ces courbes/surfaces sont extrapolables pour la fertilisation des unités de fertilisation. Les réponses observées ne dépendent toutefois pas que des doses d'engrais étudiées; elles dépendent aussi des propriétés du sol, de sa capacité à stocker et à restituer les éléments minéraux. C'est pourquoi l'étude spatiale des réponses des teneurs au sein des unités de sol est un outil aussi important que le traitement mathématique des résultats de l'expérience. Il s'agit de vérifier qu'on observe pour les unités de fertilisation de la plantation, des réponses des teneurs foliaires aux applications d'engrais, conformes aux réponses obtenues expérimentalement.

\section{Apprendre davantage des résultats des essais}

Un essai ne sert pas uniquement à déterminer des teneurs foliaires optimales. C'est un lieu de référence homogène où les pratiques culturales sont bien contrôlées (entretien, fertilisation, récolte). L'essai permet de répondre parfois à des questions qui émergent de la plantation où les données ne pas organisées pour émettre des hypothèses explicatives. Voyons quelques exemples. 
Sur une large partie de la plantation, on peut observer une variation inattendue, et d'une amplitude peu commune, des teneurs en $\mathrm{N}, \mathrm{K}$ ou Mg. L'essai est évidemment le meilleur outil pour vérifier si cette variation concerne toutes les doses testées lorsque l'élément fait partie des facteurs étudiés. Quelques exemples ont montré que ces évènements étaient souvent indépendants de la fertilisation. En conséquence, on n'appliquera pas intégralement la table de fertilisation si les résultats expérimentaux confirment que les variations de teneurs sont dues à d'autres facteurs que la fertilisation.

De la même manière, un essai est un bon témoin lorsqu'on observe une baisse de rendement, lors d'une année particulière ou sur plusieurs années consécutives. On peut examiner le rôle des éléments testés par le protocole. On dispose aussi d'une information très précise sur les composantes du rendement (nombre de régimes et poids moyen). Cependant, on ne formulera pas les mêmes hypothèses sur les mécanismes à l'origine de la baisse de rendement : une baisse du nombre de régime est en général consécutive à un stress, alors qu'une baisse du poids moyen des régimes peut provenir d'un défaut de pollinisation (figure 3).

Les essais nous renseignent aussi sur la résilience des réserves des sols et le risque d'apparition d'une déficience. Au cours des premières années des essais, on ne détecte, souvent, aucun effet positif ou négatif des facteurs étudiés (en particulier des apports d'engrais), sur le rendement.

Dans l'exemple de la figure 29, la nutrition potassique de la modalité K0 (sans apport d'engrais $\mathrm{KCl}$ ) s'est dégradée lentement; ce n'est qu'à partir de 9 ans que des différences significatives de rendement sont enregistrées par rapport à K1 et K2. Selon la nature des sols, les délais de mise en place des déficiences sont souvent compris entre 5 et 10 ans pour les témoins non fertilisés en $\mathrm{N}$ ou en K (Dubos et Flori, 2014). Pour ces raisons, dans une plantation industrielle fertilisée régulièrement, le risque d'induire une perte de rendement consécutive à une recommandation d'engrais sous-évaluée est très faible, puisque l'on observe la dégradation des teneurs avant qu'elle ne se répercute sur les rendements. Les essais fournissent aussi une bonne estimation des réserves disponibles pour les cultures les plus âgées dont la fertilisation est suspendue 2 à 3 ans avant leur replantation. 



\section{4 \\ Extrapolation de l'application des tables de fertilisation issues des essais}

Il s'agit de déterminer les périmètres géographiques sur lesquels les résultats des tables de fertilisation sont applicables. Les essais de fertilisation fournissent les courbes de réponse des rendements et des teneurs foliaires aux apports d'engrais. En identifiant les seuils de déficience et les teneurs optimales, on crée des tables de fertilisation qui sont adaptées aux conditions locales de sol et de climat.

Il faut vérifier que les teneurs optimales et les tables de fertilisation obtenues sont valides pour toute la plantation. Dans ce but, la caractérisation des sols de la plantation et l'analyse spatiale des données parcellaires sont des moyens puissants pour détecter les zones de la plantation qui font exception. Il faudra ajuster les tables de fertilisation pour ces secteurs et cela peut nécessiter la mise en place de nouvelles expériences.

\section{Analyser le comportement de la plantation à l'échelle des unités de fertilisation}

L'origine du matériel végétal et la nature des sols sont les principaux facteurs qui peuvent avoir une influence sur l'allocation des éléments dans les folioles puisque les données climatiques (pluviométrie et ensoleillement) ne varient pas suffisamment pour influencer la nutrition minérale à l'intérieur d'une plantation.

La variabilité des teneurs foliaires en relation avec le matériel végétal est pour l'instant peu documentée. Cependant on a établi qu'à fertilisation équivalente les teneurs foliaires en $\mathrm{N}$ et $\mathrm{K}$ de E. oleifera $\times$ E. guineensis sont plus faibles de celles de E. guineensis sur un même site, en raison de biomasses des feuilles et des troncs différentes. Dès que les surfaces plantées deviennent importantes (par exemple supérieures à 1000 ha) il faut mettre en place des essais pour chaque type/origine génétique de matériel végétal. La même précaution vaut pour les croisements dont au moins un des parents a une origine génétique reconnue différente du reste du matériel végétal (par exemple entre La Mé et Yangambi pour E. guineensis, et entre Coari et Manicoré pour E. oleifera). Les propriétés chimiques des sols peuvent modifier les teneurs foliaires par des mécanismes qui ne sont pas encore bien compris mais dont les conséquences peuvent être 
importantes pour le potassium en particulier (section «Tenir compte des teneurs en Ca du sol» page 65). Dès que l'on dispose de données (carte pédologique, carte topographique) qui montrent que de grandes unités de sol (par exemple des dépressions alluviales et des plateaux de roches sédimentaires) ont des propriétés texturales et chimiques différentes, il est indispensable de concevoir des essais de fertilisation spécifiques.

\section{Quelles informations tirer des analyses de sol?}

On ne dispose pas toujours d'une carte des sols détaillée, mais il est courant d'analyser quelques échantillons composites avant de planter les premiers palmiers. Ces échantillons sont généralement représentatifs des principales unités de sol et ils apportent deux types de renseignements utiles :

- les éléments ( $\mathrm{N}, \mathrm{P}, \mathrm{K}$ et $\mathrm{Mg}$ pour les principaux) dont les réserves édaphiques sont faibles et pour lesquels des déficiences peuvent apparaître dès les premières années de culture. Les premiers barèmes de fertilisation prendront en compte cet état des lieux; - les propriétés qui influent la capacité du sol à stocker et restituer l'eau et les éléments minéraux; il s'agit principalement du taux de matière organique (MO), de la capacité d'échange de cations (CEC) et de la texture. Si ces propriétés sont sous-optimales, on cherchera à améliorer le fonctionnement du sol par des pratiques culturales adaptées, comme l'installation d'un couvert de légumineuses et des apports de matière organique (voir section "Améliorer les propriétés physico-chimiques des sols» page 78). Les analyses de sol avant plantation vont permettre de détecter une probable déficience en phosphore. Les seuils de référence en dessous desquels on obtient une réponse aux engrais phosphatés varient selon les méthodes d'analyse (d'après Caliman et al., 1994) :

- P total 400 ppm;

- P Saunders 130 ppm;

- P Olsen 30 ppm;

- P Bray n 215 ppm.

Lorsque les résultats des analyses sont inférieurs à ces seuils, on peut appliquer systématiquement des phosphates jusqu'à ce que des résultats expérimentaux définissent les teneurs foliaires optimales et les doses d'apport recommandées. Il arrive qu'une partie du P apporté soit fixé sous une forme peu soluble, en particulier lorsque le $\mathrm{pH}$ du sol est acide et en présence d'aluminium et d'oxydes de fer. Il faut d'abord saturer le pouvoir fixateur du sol avant d'obtenir des réponses des teneurs et des rendements aux apports d'engrais. Seuls des essais de fertilisation permettent de faire de telles observations et préciser la rentabilité de la fertilisation en P dans ces conditions.

Les valeurs des teneurs du sol en cations échangeables (principalement $\mathrm{K}^{+}$et $\mathrm{Mg}^{++}$) ne donnent pas toujours d'indications fiables pour détecter une déficience et ici encore la méthode d'analyse est importante. Les réserves en $\mathrm{K}$ et en $\mathrm{Mg}$ échangeables sont considérées comme insuffisantes lorsqu'elles sont inférieures à $0,2 \mathrm{cmol} / \mathrm{kg}$, mais ce seuil se réfère à une extraction à l'acétate d'ammonium qui est inadaptée aux sols tropicaux acides. Ainsi, Foster et Prabowo (1996) ont montré que l'extraction de K avec du $\mathrm{HCl}$ bouillant donnait une estimation des réserves en $\mathrm{K}$ cohérente avec les 
réponses du rendement observées dans un réseau d'essais en Indonésie, alors que l'extraction à l'acétate d'ammonium ne permettait pas d'expliquer les résultats. Il est donc recommandé de suivre une méthode pour laquelle le $\mathrm{pH}$ de la solution d'extraction (cobalt hexamine trichloride ou chlorure de barium) est proche du $\mathrm{pH}$ du sol. Même avec cette précaution, l'analyse du K échangeable ne donne pas toujours une estimation correcte de ce qui est disponible pour les palmiers. Ainsi, dans des sols alluviaux en Colombie, Dubos et al. (2011) ont conclu que les réserves en $\mathrm{K}$ devaient être beaucoup plus élevées que l'indiquait l'extraction par le cobalt hexamine trichloride (teneurs inférieures à $0,2 \mathrm{cmol} / \mathrm{kg}$ en l'absence d'apport de K). Cette conclusion a été établie en faisant un bilan du potassium des parties aériennes (tableau 6).

Tableau 6. Masses de $\mathrm{K}$ et de $\mathrm{Cl}$ ( $\mathrm{kg} /$ palmier) mesurés dans des palmiers de type guineensis de 11 ans en Colombie.

\begin{tabular}{lcccccc}
\hline \multirow{2}{*}{ Organe du palmier } & \multicolumn{3}{c}{ Stock de Cl $\mathbf{( k g}$} & \multicolumn{3}{c}{ Stock de K $(\mathbf{k g})$} \\
\cline { 2 - 7 } & T0 & TKCl & TNaCl & T0 & TKCl & TNaCl \\
\hline Tronc & 0,48 & 1,76 & 1,91 & 3,27 & 4,10 & 4,51 \\
\hline Couronne de feuilles & 0,39 & 1,23 & 1,22 & 1,37 & 1,46 & 1,44 \\
\hline Total & 0,87 & 2,99 & 3,13 & 4,64 & 5,56 & 5,96 \\
\hline
\end{tabular}

Les quantités ont été calculées à partir d'une biomasse du tronc de $150 \mathrm{~kg}$ MS et de 35 feuilles dans la couronne. Le chlore est apporté soit sous forme de $\mathrm{KCl}(\mathrm{TKCl})$ soit sous forme de $\mathrm{NaCl}(\mathrm{TNaCl})$ à hauteur de $2 \mathrm{~kg} \mathrm{Cl} /$ palmier/an. En comparaison au témoin sans apport (T0), le stock de $\mathrm{Cl}$ a plus que triplé avec $\mathrm{TKCl}$ et $\mathrm{TNaCl}$, et cela s'accompagne d'une hausse du stock de $\mathrm{K}$. Pour $\mathrm{TNaCl}$, le stock de $\mathrm{K}$ augmente de $28 \%$ par rapport au témoin et ce $\mathrm{K}$ ne peut provenir que des réserves du sol dont l'analyse des sols indique qu'elles sont faibles.

En définitive, les analyses de sol ne donnent qu'une information assez limitée sur les besoins en engrais. Comme elles ne sont pas bien corrélées avec les apports de fertilisants et avec les rendements, elles ne peuvent pas servir directement d'outil de pilotage de la fertilisation. Par contre, elles fournissent des informations essentielles pour comprendre les réponses des teneurs foliaires et le fonctionnement de l'outil de diagnostic foliaire. On a vu que le calcium n'était pas un élément important pour le palmier à huile. Cependant l'abondance du calcium dans le sol peut interférer avec l'allocation du potassium dans les folioles et biaiser les teneurs analysées. Or la fertilisation potassique représente la part la plus importante des apports d'engrais. Ceci justifie que l'on traite séparément cette question spécifique aux apports de $\mathrm{KCl}$.

\section{Tenir compte des teneurs du sol en calcium lorsqu'on utilise du $\mathrm{KCl}$}

Le potassium est l'élément majeur de la fertilisation du palmier à huile et les corrélations obtenues entre les rendements et les teneurs foliaires ont beaucoup contribué au succès du diagnostic foliaire. Le potassium est aussi l'élément pour lequel on a, dans certaines circonstances, rencontré des difficultés pour préconiser, les quantités d'engrais à appliquer. Ces difficultés fournissent un bon exemple d'interaction entre les propriétés du sol et l'allocation des éléments dans les folioles. 
Dubos et al. (2017a) ont étudié les réponses des teneurs foliaires en $\mathrm{K}$ aux apports de $\mathrm{KCl}$ en utilisant les résultats de 13 essais de fertilisation répartis dans le monde, situés sur différents types de sol. Ils ont classé les réponses observées en trois catégories (figure 31) : la teneur en K augmente avec l'apport d'engrais (réponse positive, c'est le cas de la majorité des essais), la teneur ne varie pas (réponse neutre), la teneur diminue avec l'apport (réponse négative).

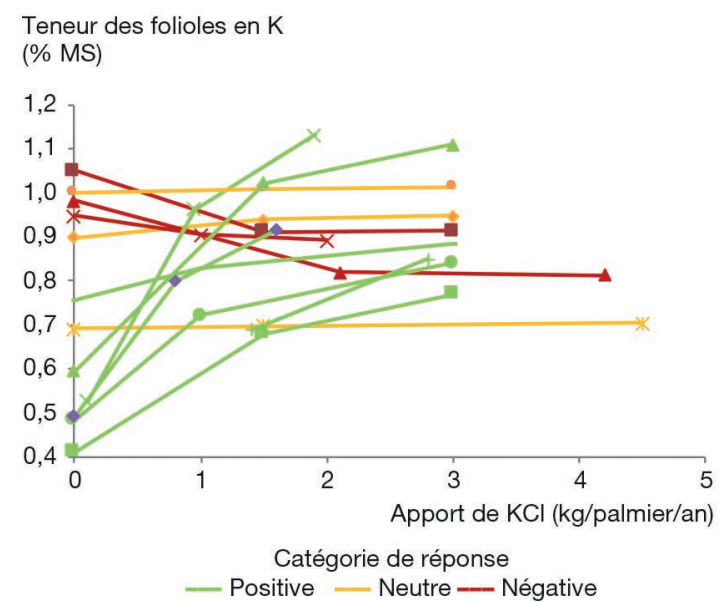

Figure 31. Trois catégories de réponse des teneurs en $\mathrm{K}$ des folioles aux apports de $\mathrm{KCl}$ (essais situés en Afrique, en Asie et en Amérique latine).

Pour 7 essais sur 13, les teneurs augmentent avec l'apport (traits verts); pour 3 essais, il n'y a pas de réponse (traits jaunes); pour 3 essais, les teneurs baissent (traits rouges). Les données de ces essais sont reprises pour élaborer la figure 32.

Lorsque les teneurs foliaires en $\mathrm{K}$ ne varient pas ou diminuent, il est impossible de déterminer une teneur optimale en $\mathrm{K}$ par la méthode des courbes de réponse décrite à la section "Déterminer la plage des teneurs optimales pour un élément» page 56. La propriété des sols la mieux corrélée à ce dysfonctionnement est la "pression calcique» exprimée par le rapport (en \%) entre la teneur du sol en Ca échangeable et la capacité d'échange cationique (CEC). Ce rapport permet de prédire l'augmentation des teneurs foliaires en $\mathrm{K}$ par rapport à un témoin non fertilisé en $\mathrm{KCl}$ (figure 32).

Dans deux des expériences qui ont servi à établir ces relations Dubos et al. (2011) ont montré que bien que les teneurs foliaires en $\mathrm{K}$ aient diminué avec les doses de $\mathrm{KCl}$ cela ne remettait pas en cause l'absorption du K par les palmiers puisque les teneurs des troncs et des rachis des feuilles augmentaient (figure 33).

Lorsque la pression calcique du sol est élevée, on observe que, concomitamment à la baisse des teneurs foliaires en $\mathrm{K}$ avec les apports de $\mathrm{KCl}$, les teneurs en $\mathrm{Ca}$ augmentent. Ce phénomène appelé antagonisme K-Ca met aussi en jeu la synergie $\mathrm{Cl}-\mathrm{Ca}$ que l'on observe en appliquant du $\mathrm{NaCl}$. Il ne s'agit pas d'un antagonisme pour l'absorption de Ca préférentiellement à $\mathrm{K}$ par le palmier mais plutôt pour l'allocation de $\mathrm{Ca}$ préférentiellement à $\mathrm{K}$ dans la foliole. Les déterminants de l'allocation des cations 


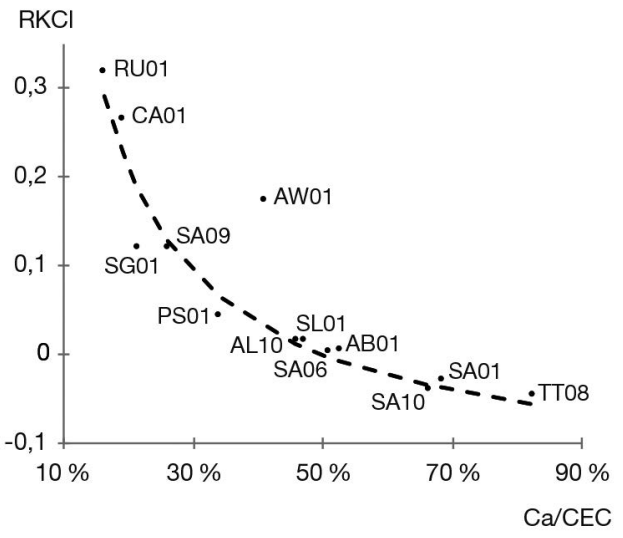

Figure 32. Courbe de réponse des teneurs en potassium en fonction de la pression calcique (d'après Dubos et al. 2017a).

À partir des données de la figure 31, on a calculé la réponse $\mathrm{R} \mathrm{KCl}$ qui exprime la variation de teneur en $\mathrm{K}(\% \mathrm{MS})$ par $\mathrm{kg}$ de $\mathrm{KCl}$ appliqué, entre la modalité la plus élevée en $\mathrm{KCl}$ et la modalité sans $\mathrm{KCl}$. Lorsque la "pression calcique», c'est-à-dire le ratio Ca/CEC (teneur en $\mathrm{Ca}$ échangeable du sol divisée par la capacité d'échange cationique), atteint puis dépasse $50 \%$, les réponses à l'engrais $\mathrm{KCl}$ des teneurs foliaires en $\mathrm{K}$ deviennent neutres $(\mathrm{R} \mathrm{KCl}=0)$ puis négatives.

Teneur des folioles en $\mathrm{K}$

(\% MS)

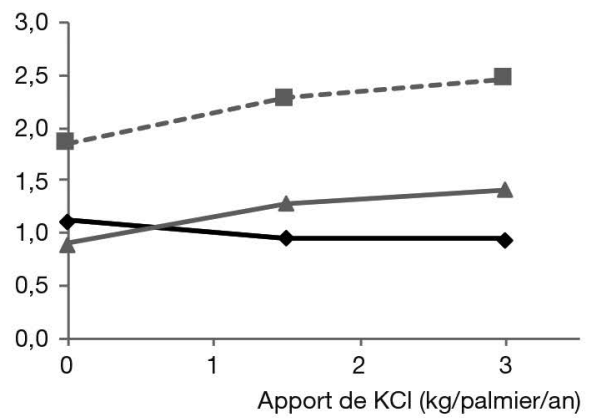

A $\longrightarrow$ Folioles

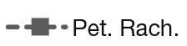

$\longrightarrow$ Tronc
Teneur des folioles en $\mathrm{K}$ (\% MS)

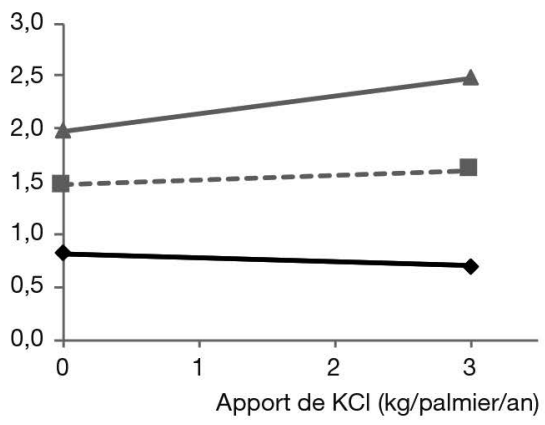

B $\longrightarrow$ Folioles $-\rightarrow \cdot$ Pet. Rach. $\longrightarrow$ Tronc

Figure 33. Teneurs en potassium observées dans des essais en Équateur (figure 33A) et en Colombie (figure 33B), à 10 et 11 ans.

Dans ces deux plantations les analyses indiquent que les apports d'engrais $\mathrm{KCl}$ diminuent significativement les teneurs en $\mathrm{K}$ des folioles. Cependant, l'augmentation des teneurs des troncs et du composite pétiole + rachis (Pet. Rach.) de la feuille 17 montre qu'il y a bien eu absorption du potassium par les racines et migration jusqu'au rachis des feuilles. 
n’ont pas été décrits avec précision mais il en résulte que lorsque la pression calcique est élevée, les teneurs foliaires en $\mathrm{K}$ peuvent stagner dans des plages de valeurs de teneurs foliaires faibles $(0,70-0,80 \% \mathrm{MS})$ sans que l'on puisse parler de déficience puisque cela ne diminue pas les rendements. Cependant ces conditions de sols ne permettent pas de déduire une recommandation correcte d'apport d'engrais $\mathrm{KCl}$ pour améliorer les teneurs des folioles en $\mathrm{K}$.

\section{Comment détecter les perturbations dues à des propriétés des sols?}

Dans l'exemple précédent, la méthode la plus sûre consiste à s'appuyer sur des analyses de sol qui permettent de détecter les secteurs de la plantation où le rapport $\mathrm{Ca} / \mathrm{CEC}$ dépasse $50 \%$. Cependant, lorsqu'on dispose d'une carte des sols, la description des unités de sol ne repose que sur un nombre limité d'analyses. Or on a besoin d'examiner le rapport $\mathrm{Ca} / \mathrm{CEC}$ du sol de chaque unité de fertilisation sur le site de prélèvement des folioles pour le diagnostic foliaire.

Lorsqu'on a identifié des secteurs où le rapport $\mathrm{Ca} / \mathrm{CEC}$ est suffisamment élevé pour fausser l'allocation de $\mathrm{K}$ dans les folioles, il faut ensuite confirmer que les teneurs foliaires ne répondent pas aux apports de $\mathrm{KCl}$. L'analyse du comportement des unités de fertilisation avec des outils cartographiques permet de confirmer l'existence de conditions de sol qui altèrent l'allocation du potassium sans pour autant avoir de conséquences sur les rendements. Ces outils sont applicables pour n'importe quel élément dont on cherche à établir que les recommandations d'engrais ont réellement un effet sur les teneurs et lorsqu'on cherche à préciser l'ampleur de cet effet (section " construire un système d'information géographique»).

Enfin on peut mettre en place des tests de réponse simples pour confirmer qu'il existe des problèmes d'interprétation des résultats de diagnostic foliaire qui sont localisés sur certaines zones. Ils sont à la fois moins coûteux et plus rapides que la mise en place d'un véritable essai de fertilisation (section «Mettre en place des tests de réactivité» page suivante).

\section{Associer une analyse de référence du sol à chaque échantillon foliaire}

Il est recommandé de restreindre la surface couverte par l'échantillon foliaire à une partie de l'unité de fertilisation occupée par une seule unité de sol (section "choisir les palmiers pour l'échantillon foliaire de référence» page 42). Il est donc tout à fait légitime de disposer d'une analyse de référence pour ce sol. Cela permet de comprendre les réponses des teneurs aux engrais lorsque l'on soupçonne des dysfonctionnements tels que nous venons de le voir précédemment. On pourra alors se doter de jeux de données (teneurs foliaires, épandages d'engrais et propriétés des sols) suffisants pour envisager une analyse multivariée et identifier des relations explicatives pour préciser les teneurs optimales en fonction des sols.

L'analyse de sol de référence est assimilée à un jeu de données descriptives du sol de chaque unité de fertilisation. Il n'est pas justifié de la répéter dans le temps (un prélèvement par cycle) puisqu'elle n'est pas destinée à un suivi des réserves des sols (sujet abordé en section 
«Évaluer les réserves du sol»). Elle ne doit pas non plus être soumise à des variations de teneurs qui seraient dues à des pratiques de fertilisation : le prélèvement se fera donc à l'extérieur des zones de fertilisation minérale ou organique. Lorsque la culture est déjà en place depuis au moins une dizaine d'années, les zones d'accumulation des feuilles d'élagage sont à éviter parce que le sol peut être enrichi en cations par recyclage de la biomasse. En définitive, il est préférable d'analyser des échantillons composites de sol le plus tôt possible, soit à 3 ans lors de la mise en place de l'échantillon foliaire pour l'unité de fertilisation. L'échantillonnage du sol très localisé dont le meilleur exemple est le dispositif en paire de lignes (figure 23, UDF02) est recommandé pour obtenir une référence qui sera associée à un objet ponctuel dont on pourra préciser les coordonnées géographiques : chaque palmier de l'échantillon peut être associé à un prélèvement élémentaire de sol pour constituer le composite. Une profondeur de 30 centimètres est suffisante pour caractériser la couche superficielle où sont concentrées les racines des surfaces situées à plus de de 2 mètres de distance du tronc. Les horizons sous-jacents peuvent aussi être prélevés sur la même épaisseur jusqu'à 90 centimètres si l'on souhaite disposer de plus d'informations sur les sols de la plantation.

\section{Construire un système d'information géographique (SIG)}

Pour comprendre finement les relations entre les propriétés des sols et les teneurs foliaires, indépendamment des doses d'engrais appliquées, il faudrait tester expérimentalement de nombreux sols au sein de la plantation et ceci pour plusieurs éléments (P, K et Mg en priorité). Cette méthode est longue et onéreuse. Par contre, en examinant les réponses des teneurs foliaires des unités de fertilisation on peut détecter les lieux où on n'atteint pas la teneur que l'on avait prévue en utilisant le barème de fertilisation. Si on dispose d'un SIG, chaque point d'observation de diagnostic foliaire peut être reporté et on va chercher à comprendre si les valeurs les plus faibles et les plus fortes sont réparties aléatoirement ou regroupées géographiquement. Il peut être nécessaire de tenir compte de l'âge comme présenté en figure 34 qui décrit les teneurs foliaires moyennes en magnésium entre 8 et 12 ans. On peut aussi cartographier les propriétés chimiques du sol des unités de fertilisation et déterminer si les secteurs où le rapport $\mathrm{Ca} / \mathrm{CEC}$ est plus élevé, se superposent avec les teneurs en $\mathrm{K}$ les plus faibles au même âge.

L'analyse spatiale permet de superposer plusieurs couches d'informations (carte géologique, carte pédologique, carte topographique, carte des réserves en eau du sol, profondeur de nappe, matériel végétal). Cette approche est très utile pour émettre des hypothèses, non seulement sur ce qui perturbe les teneurs foliaires de n'importe quel élément, mais aussi sur le rôle d'autres variables (état sanitaire par exemple) pour expliquer des variations de rendements.

\section{Mettre en place des tests de réactivité}

Le rachis des feuilles est un tissu de référence pour tester l'absorption du potassium car ses teneurs augmentent avec l'application de $\mathrm{KCl}$, y compris lorsque les teneurs des folioles diminuent avec les apports (exemple figure 33). Ces réponses contradictoires permettent de mettre en place des tests de réactivité pour confirmer que dans 


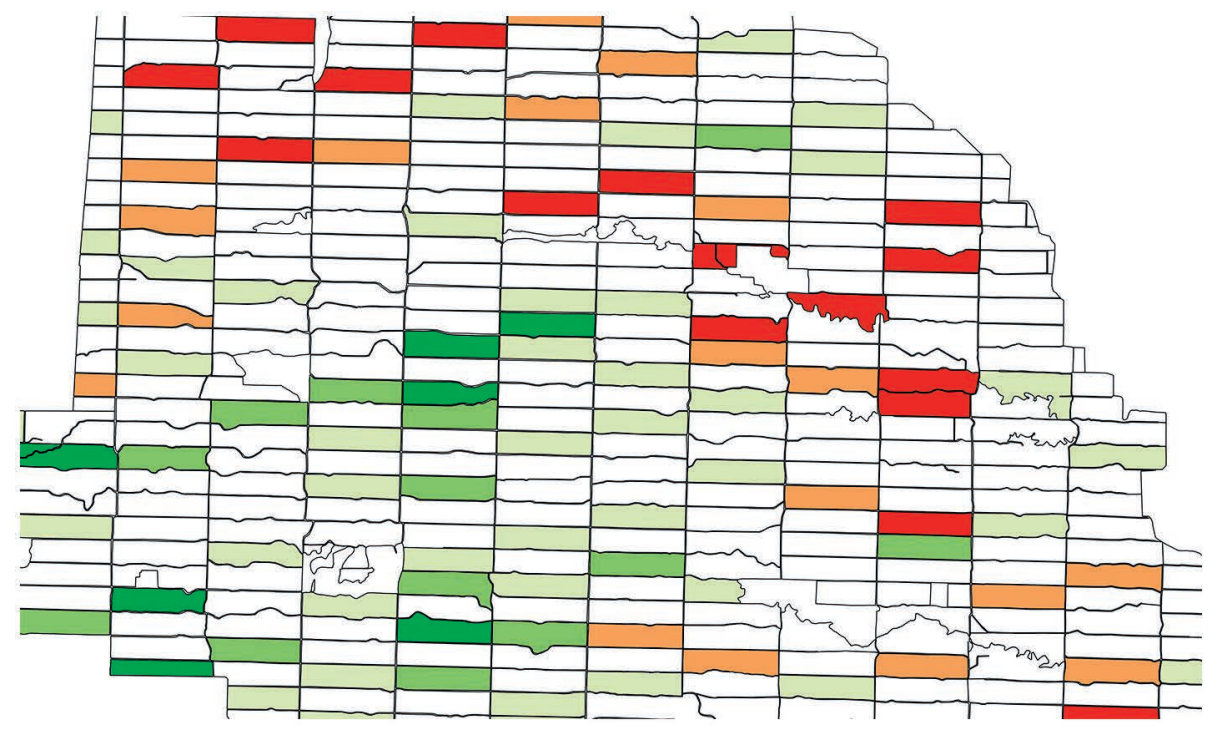

Gammes des teneurs en magnésium (moyennes de 8 à 12 ans)

$\square$ 0,00-0,18 $\square 0,18-0,20 \square 0,20-0,23 \square 0,23-0,27 \square 0,27-0,32$

Figure 34. Cartographie des teneurs foliaires moyennes en Mg entre 8 et 12 ans.

Les teneurs de chaque unité de fertilisation ont été reportées sur la parcelle où est prélevé l'échantillon. Quelques analyses de sol ont confirmé que les teneurs en Mg élevées (sudouest de la plantation) coïncidaient avec des propriétés chimiques différentes de celles des autres unités de sol. Là où les teneurs sont les plus faibles, des tests d'absorption ont confirmé qu'il y avait un blocage dans la translocation de $\mathrm{Mg}$ jusqu’à la foliole.

certaines unités de fertilisation les teneurs en $\mathrm{K}$ que l'on vise ne pourront pas être atteintes quelle que soit la dose de $\mathrm{KCl}$. Ces tests sont plus rapides et moins coûteux que le recours à un essai standard (encadré 9).

Les conditions pour lesquelles on ne peut plus utiliser correctement l'outil de diagnostic foliaire ne sont pas fréquentes et elles concernent principalement le potassium. Lorsque cela se produit il faut trouver une autre solution pour établir une recommandation d'engrais. Foster et Prabowo (1996) ont proposé d'utiliser l'analyse des rachis pour établir un diagnostic; ils considèrent qu'une teneur en $\mathrm{K}$ est correcte (absence de déficience ayant un impact sur le rendement) lorsqu'elle est supérieure à 1\% MS. On pourrait donc conclure que pour les deux essais présentés en figure 33, l'état nutritionnel à 10 et 11 ans était satisfaisant sans aucun apport de $\mathrm{KCl}$.

Une autre alternative consiste à définir une dose de maintenance, qui peut être établie à partir des teneurs en chlore lorsqu'il est nécessaire de contrôler la nutrition chlorée. Cette dose peut aussi être calculée pour compenser tout ou partie des exportations du potassium par les régimes mais cette estimation requiert des informations précises sur leur composition, qui ne sont pas toujours disponibles. Elle doit aussi être modulée en fonction des rendements potentiels de chaque unité de fertilisation 
ce qui n'est pas toujours simple à préciser. On recommande donc, pour des raisons tant économiques qu'environnementales de ne pas appliquer de doses supérieures à $1,5 \mathrm{~kg} \mathrm{KCl} /$ palmier/an.

\section{Encadré 9. Les tests de réactivité pour tester rapidement la sensibilité des teneurs foliaires dans différentes zones de la plantation.}

Le dispositif le plus simple consiste à délimiter des parcelles expérimentales d'environ 20 palmiers utiles entourés de palmiers de bordure et à appliquer deux niveaux de $\mathrm{KCl}$ (un témoin sans engrais et $3 \mathrm{~kg} / \mathrm{palmier} / \mathrm{an})$, avec 3 répétitions, soit 6 parcelles expérimentales au total (figure 35). On prélève des échantillons foliaires avant la première application des traitements puis 2 mois après épandage du $\mathrm{KCl}$ pendant trois ans. Il faut analyser à la fois les folioles et les rachis des feuilles et observer les résultats pour chaque organe. $\mathrm{Si}$ aucune évolution favorable des teneurs foliaires de la modalité fertilisée n'est détectée, il est probable que la translocation de $\mathrm{K}$ dans la foliole ne se fait pas correctement.

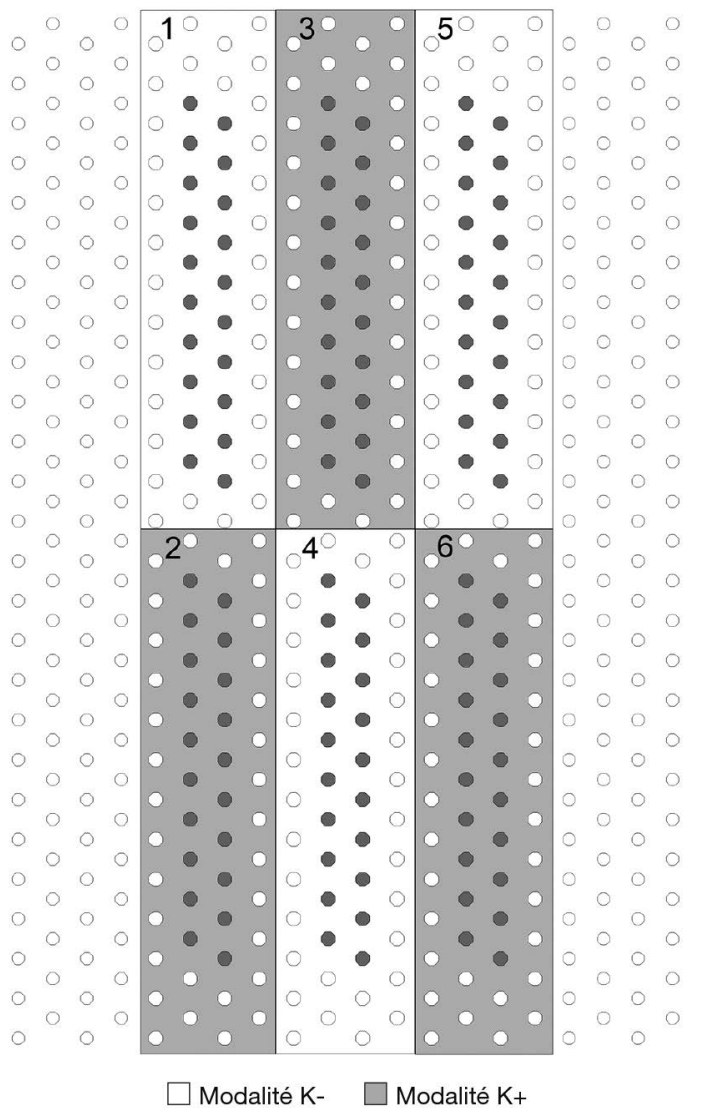

Une zone homogène d'une parcelle a été isolée pour délimiter au champ 6 parcelles expérimentales constituées de 4 lignes de 13 palmiers. Les parcelles expérimentales 1 et 2 , 3 et 4 , et, 5 et 6 , forment 3 répétitions. Tous les individus de chaque parcelle expérimentale reçoivent, après tirage au sort, une des deux modalités $(\mathrm{K}+: 3 \mathrm{~kg} \mathrm{KCl} / \mathrm{an}, \mathrm{K}-$ : non fertilisé en $\mathrm{KCl})$. Des échantillons foliaires sont prélevés en utilisant les arbres utiles (au centre) et les teneurs foliaires sont suivies pendant trois ans.

Figure 35. Exemple de test de réactivité des teneurs foliaires en $\mathrm{K}$. 



\section{5 \\ Adopter des pratiques de fertilisation durables : perspectives et recommandations}

Disposer d'un outil d'aide à la décision pour la nutrition des palmeraies paramétré selon les conditions locales permet d'appliquer les doses d'engrais optimales pour atteindre le rendement potentiel tout en maîtrisant le coût de ce facteur de productivité. La démarche est pleinement efficace lorsque les engrais épandus dans la plantation produisent les mêmes effets que dans les essais et qu'ils permettent d'atteindre les teneurs foliaires recommandées. Les conditions de travail dans les essais sont nettement plus homogènes et contrôlées que dans les unités de fertilisation, il faut donc adopter les pratiques qui permettent une absorption optimale des éléments minéraux en tout lieu de la plantation. Si ce n'est pas le cas, il faudra recourir à doses d'engrais plus importantes qu'initialement prévu. Les conséquences économiques ne constituent pas le seul enjeu de la fertilisation raisonnée, puisque les quantités d'éléments non prélevées par la culture peuvent générer des effets indésirables sur la santé des sols et la qualité des ressources en eau.

Investir dans un outil d'aide à la décision précis n'a donc de sens que si l'on adopte les meilleures pratiques culturales.

\section{Préserver la santé des sols}

Initialement, la fertilisation du palmier à huile a surtout été vue comme un moyen de maximiser les rendements. Avec l'augmentation constante du coût des intrants, issus de l'extraction minière et de la chimie, des considérations économiques ont ensuite été introduites, l'objectif étant d'atteindre des rendements élevés à un coût de fertilisation acceptable. Aujourd'hui, il est indispensable de viser un objectif beaucoup plus ambitieux qui consiste à concilier l'intensification agronomique avec l'optimisation écologique. Comme on l'a rappelé en début d'ouvrage, les propriétés physico-chimiques des sols conditionnent largement le rendement potentiel de chaque plantation. Faire en sorte que la fertilisation n'altère pas la capacité des sols à assurer les services écosystémiques permettant une alimentation correcte en eau et en nutriments doit être une préoccupation à long terme. Il faut donc s'interroger sur les conséquences de recommandations d'engrais déséquilibrées par rapport à la demande de la culture : cela pourrait-il modifier sur les propriétés du sol fertilisé? Inversement peut-il y avoir un appauvrissement progressif des sols qui compromette leur durabilité? 


\section{Se soucier de la fertilité chimique des sols}

L'objectif est de s'assurer qu'il n'y a pas d'évolution défavorable des propriétés des sols en raison de l'accumulation d'un élément minéral dans le sol. Ce déséquilibre peut favoriser l'appauvrissement du sol en d'autres éléments et modifier d'autres propriétés comme le $\mathrm{pH}$ et la CEC du sol. Quelques exemples ont été documentés.

En Côte d'Ivoire, dans la savane de Dabou, après un premier cycle de culture, les palmeraies ont présenté des rendements inférieurs à ceux du cycle précédent au même âge. Ce phénomène n'était pas dû à des pratiques culturales différentes, en particulier la fertilisation; les études ont révélé que l'alimentation hydrique des palmiers en replantation avait diminué par rapport à celle du premier cycle en raison de la déstructuration et de la compaction de l'horizon de surface (Caliman et al., 1987). Ce changement dû à des passages répétés d'engins lors de la préparation du terrain a été amplifié par les apports potassiques répétés. Ainsi, l'enrichissement en K échangeable de l'horizon de surface a contribué à la solubilisation et à la précipitation des hydroxydes de fer et d'aluminium puis à la cimentation des particules de sable.

En Équateur, une étude a été entreprise à partir de deux essais factoriels testant l'apport de $\mathrm{N}$ et de $\mathrm{K}$ à différentes doses (Dubos et al., 2017b). L'analyse du sol sur lequel les engrais ont été appliqués pendant 10 ans a mis en évidence une évolution défavorable des propriétés des sols lorsque les doses sont en excès par rapport à la capacité de stockage du sol ou aux prélèvements de $\mathrm{N}$ et de $\mathrm{K}$, par la culture. Les apports azotés (urée) ont conduit à une acidification du sol que l'on explique par le lessivage de l'azote en excès sous forme de nitrates. En migrant vers la profondeur, les anions $\mathrm{NO}_{3}{ }^{-}$sont combinés à des cations $\mathrm{Ca}^{++}$et $\mathrm{Mg}^{++}$et cela génère un appauvrissement de l'horizon superficiel, pour ces cations bivalents (figure 36). De manière identique, de fortes doses de $\mathrm{KCl}$ ont provoqué une augmentation du K échangeable du sol (figure 37) et le déplacement des cations $\mathrm{Ca}$ et $\mathrm{Mg}$ par remplacement sur les sites de fixation de la CEC.

Ces exemples montrent que l'enrichissement en éléments de la couche superficielle du sol peut avoir des conséquences défavorables en modifiant les propriétés du sol et en favorisant les pertes en éléments minéraux. Pour diminuer ces risques, on doit éviter à la fois une recommandation d'apport excessif d'engrais par rapport à la demande de la culture et par rapport à capacité du sol à retenir les nutriments.

\section{Évaluer les réserves des sols}

Une autre préoccupation concerne l'évolution des réserves minérales des sols à long terme. Ces réserves minérales diminuent-elles avec le temps si les apports d'engrais ne suffisent pas à compenser ce que la culture prélève?

L'organisation internationale RSPO (Roundtable on Sustainable Palm Oil) ${ }^{1}$, dans le cadre des critères de certification pour une production durable d'huile de palme, recommande un suivi périodique des propriétés des sols à l'aide d'analyses appropriées,

${ }^{1}$ RSPO : Roundtable on Sustainable Palm Oil - Table ronde sur l'huile de palme durable, https://rspo.org/ 

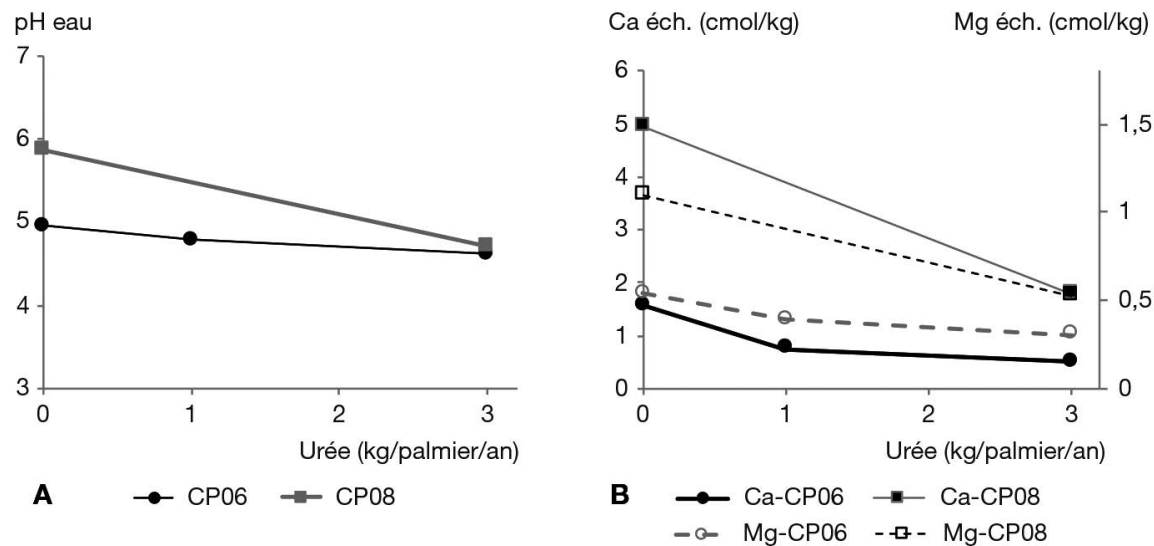

Figure 36. Mesures du pH du sol superficiel (figure 36A) et des teneurs du sol en $\mathrm{Ca}$ et Mg échangeables (figure 36B) dans deux essais factoriels (nommés CP06 et CP08) conduits pendant 10 ans en Equateur.

Le pH et les teneurs du sol en Ca et en $\mathrm{Mg}$ chutent lorsque les apports d'urée augmentent. Les concentrations en $\mathrm{H}^{+}$et $\mathrm{Al}^{3+}$, également mesurées, augmentent significativement avec l'urée. Les apports excessifs d'urée sont à l'origine d'un lessivage sous forme de nitrates qui entraîne des cations bivalents $\mathrm{Ca}^{++}$et $\mathrm{Mg}^{++}$.

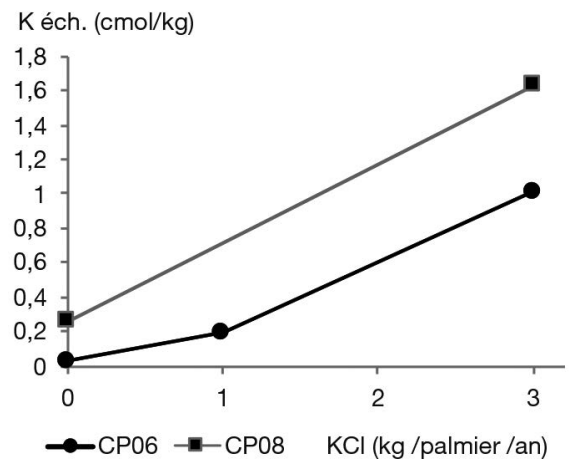

Figure 37. Mesures du K échangeable du sol en fonction de l'apport de $\mathrm{KCl}$ sur deux essais factoriels (nommés CP06 et CP08) conduits pendant 10 ans en Équateur.

Le potassium qui n'est pas absorbé par les palmiers explique les teneurs en K échangeable du sol particulièrement élevées avec l'application de $3 \mathrm{~kg} \mathrm{KCl} /$ palmier $/ \mathrm{an}$. Ce potassium en excès dans la couche superficielle est susceptible de migrer en profondeur, au-delà de la couche des racines absorbantes; il peut aussi déplacer les cations $\mathrm{Ca}$ et $\mathrm{Mg}$ de la CEC.

tous les cinq ans par exemple. L'intérêt est de maintenir les réserves du sol à un niveau suffisant pour garantir l'obtention de hauts rendements et pour préserver la fertilité du sol à la fin du cycle de culture.

Ces analyses permettent un contrôle a posteriori de l'impact de la fertilisation pilotée par le diagnostic foliaire. Il faut toutefois tenir compte de la structure des palmeraies 
car l'exploitation conduit à individualiser différents "compartiments» de sol dont les propriétés évoluent avec les pratiques qui y sont appliquées. Ces compartiments sont par exemple les ronds sarclés au pied des palmiers, les chemins de récolte, les zones de dépôt des feuilles, les surfaces fertilisées avec les engrais. Tenir compte de cette structure conduit à deux approches :

- évaluer les réserves globales du sol de la parcelle en intégrant proportionnellement chaque compartiment. Nelson et al. (2015) proposent une méthode simple de prélèvement d'échantillons composites de sol analysés périodiquement. Cette méthode semble bien adaptée aux pas de temps longs (10 ans, voire un cycle entier de culture); - se focaliser sur les compartiments les plus sensibles à l'appauvrissement, par exemple les zones non fertilisées, ou au contraire les plus sensibles à l'enrichissement, comme les zones d'épandage des engrais.

Dubos et al. (2017b), on a comparé les teneurs en $\mathrm{K}$ du sol en dehors de la zone d'application des engrais dans les parcelles expérimentales qui reçoivent ou non du $\mathrm{KCl}$ (figure 37). L'objectif était de mesurer si le fait de ne pas compenser les prélèvements en $\mathrm{K}$ par des apports de $\mathrm{KCl}$ modifiait significativement les réserves du sol par rapport aux modalités qui recevaient de l'engrais. Dans aucun des deux essais, on n'a constaté de différences significatives après 10 ans de protocole, ce qui suggère que l'appauvrissement en $\mathrm{K}$ du sol des parcelles expérimentales sans apport est resté inférieur à la précision des analyses de sol. Pour l'essai factoriel CP06, dont les réserves minérales du sol étaient faibles dès le début de l'expérience, une déficience en $\mathrm{K}$ s'est manifestée par la baisse significative des teneurs foliaires en $\mathrm{K}$ et des rendements (figure 29); pour autant, la teneur en Kéchangeable du sol de cet essai n'a pas diminué au point d'être discriminée par l'analyse.

Cet exemple montre qu'en ajustant les apports d'engrais à partir des résultats de diagnostic foliaire, on peut intervenir avant qu'un appauvrissement du sol soit décelable par l'analyse directe. Cela confère au diagnostic foliaire un rôle protecteur vis-à-vis des effets négatifs sur le sol, qualifiés de mining effects par plusieurs auteurs.

\section{Améliorer l'efficience de la fertilisation}

\section{Réduire les pertes en éléments minéraux par différentes pratiques culturales}

Les éléments apportés par les engrais demeurent à la surface du sol avant de passer progressivement dans la couche superficielle, où ils demeureront jusqu'à leur absorption par les palmiers ou les autres espèces végétales. Au cours de ce séjour plusieurs processus contribuent à des pertes en éléments qui ne bénéficieront pas aux palmiers.

\section{Volatilisation de l'azote}

Les pertes d'azote par volatilisation sont spécifiques de certains engrais comme l'urée et les nitrates d'ammonium. Elles génèrent des gaz (Ammoniac $\mathrm{NH}_{3}$ et protoxyde d'azote 
$\mathrm{N}_{2} \mathrm{O}$ ) qui sont des gaz à effet de serre contribuant au réchauffement climatique. Pour ces engrais, il faut éviter les périodes sèches pour réduire le risque de volatilisation de $\mathrm{NH}_{3}$. On recommande d'épandre les engrais en saison des pluies, mais en évitant les mois les plus pluvieux, parce que cela favorise le risque de volatilisation de $\mathrm{N}_{2} \mathrm{O}$ et de lessivage de $\mathrm{NO}_{3}{ }^{-}$et de $\mathrm{NH}_{4}{ }^{+}$.

\section{Érosion et ruissellement}

Les pertes d'éléments fertilisants se font aussi par érosion, par ruissellement de surface et par lessivage (entraînement en profondeur des cations et des nitrates, sous la couche exploitée par les racines). Les données publiées varient non seulement avec les sites de mesure (Dubos et al., 2019), mais aussi à l'intérieur des parcelles car de nombreux facteurs sont impliqués (climat, pente, couverture du sol, pratiques culturales). Plutôt que de compenser les pertes par une fertilisation difficile à évaluer, ou surévaluée par prudence, il est plus judicieux de chercher à les réduire le plus possible.

Lérosion et le ruissellement de surface dépendent principalement de la pente et de la couverture du sol. Ces phénomènes peuvent être maîtrisés par des techniques culturales adaptées (disposition des feuilles d'élagage pour couvrir le sol, gestion des recrûs herbacés, plantes de couverture) et des aménagements (terrasses individuelles ou continues, pièges à sédiments).

\section{Pertes par lessivage des éléments minéraux}

Cela concerne principalement les éléments $\mathrm{N}$ et $\mathrm{K}$, en raison des quantités consommées par la culture. Elles augmentent dans les sols sableux en raison de la CEC faible. Elles augmentent aussi avec les fortes pluies lorsque la transpiration du couvert est faible et que l'eau en excès est drainée en profondeur (Banabas et al., 2008). Des pratiques culturales adaptées réduisent le lessivage en améliorant les propriétés chimiques du sol qui contribuent à la rétention des éléments sous une forme échangeable dans la couche superficielle du sol :

- apport de matière organique pour augmenter la CEC. Il s'agit des feuilles d'élagage, et des engrais organiques (rafles, fibres, compost ou autres sources si disponibles) que l'on peut réserver aux secteurs où le sol a une texture grossière ou dont les pentes sont les plus prononcées;

- dispersion des engrais minéraux sur de larges surfaces en particulier là où le sol a une CEC élevée, c'est-à-dire là où s'accumule la matière organique;

- usage d'engrais à libération contrôlée (CRF, controlled release fertilizers) qui libèrent les éléments minéraux sans perte pendant 6 à 12 mois et permettent de réduire significativement les quantités d'intrants.

Enfin, le fractionnement des doses d'engrais est souvent recommandé même si aucune étude n'est venue confirmer l'efficacité de cette pratique. Compte tenu de la difficulté à suivre les transferts d'éléments entre le sol et les racines (en particulier $\mathrm{K}$ ), on ignore la part des éléments apportés par les engrais qui est absorbée entre deux applications et le temps de résidence des nutriments dans le sol. 


\section{Améliorer les propriétés physico-chimiques des sols}

\section{Apports de matière organique}

Le recyclage de la biomasse disponible est un moyen puissant de restaurer, conserver ou améliorer les propriétés physico-chimiques des sols et, de ce fait, de réduire les pertes d'éléments minéraux nutritifs. À l'échelle d'une plantation, la fertilisation organique améliore la fertilité des sols en induisant une augmentation du $\mathrm{pH}$ (les sols tropicaux cultivés en palmier à huile sont souvent acides), du taux de carbone organique, de la CEC, et des cations échangeables (Comte et al., 2013).

Les apports de matière organique mettent en jeu des mécanismes complexes du sol qui vont au-delà du simple apport de nutriments. Par exemple, les apports de rafles (résidus de récolte issus de l'extraction de l'huile de palme, ou Empty Fruit Bunch, EFB), modifient les communautés d'organismes vivants du sol. C'est le cas des populations de vers de terre qui ont un rôle crucial sur la structure du sol, en fragmentant et redistribuant la matière organique en profondeur, et en augmentant l'aération et l'infiltration de l'eau.

Les apports organiques favorisent le développement des racines fines et absorbantes des palmiers; couplé à un effet positif sur la CEC du sol, ce type de traitement crée des conditions favorables à l'efficience de la fertilisation. Sur le long terme, les apports de matière organique augmentent durablement le potentiel de production de biomasse végétative et de régimes en régulant la nutrition hydrique et minérale de la plante. Cet effet peut être important lorsque le climat présente une saison sèche marquée.

\section{Favoriser la fixation de l'azote atmosphérique}

Un moyen efficace de générer de la matière organique recyclable consiste à cultiver des plantes de couverture à base de légumineuses (Pueraria sp., Centrosema sp., Mucuna sp., etc.). Cette pratique est commune en jeune culture, car elle protège le sol de l'érosion et facilite le contrôle des espèces adventices, en particulier des graminées. Les légumineuses contribuent aussi au bilan azoté grâce aux bactéries fixatrices de l'azote atmosphérique (notamment le genre Rhizobium) qui forment des nodules sur leurs racines. Il est parfois très difficile de maintenir cette couverture lorsque la palmeraie se referme et que la lumière sous la canopée, diminue. À l'âge adulte, à partir de 12 ans, la densité résiduelle de Pueraria sp. varie considérablement d'une plantation à l'autre et ces différences de comportement mériteraient d'être mieux comprises. Il ne s'agit probablement pas que d'une relation simple avec l'interception de la lumière par la canopée puisque les régions où il est possible de maintenir une couverture végétale coïncident parfois avec des zones peu ensoleillées (Ouest de l'Équateur, Afrique de l'Ouest par exemple). Lorsqu'il est difficile de maintenir une couverture de Pueraria sp., on peut semer des espèces tolérantes à l'ombrage comme Desmodium ovalifolium.

\section{Augmenter la biomasse recyclable}

Laisser pousser des recrûs de petits ligneux en culture adulte est aussi une voie intéressante pour augmenter la biomasse recyclable. L'entretien des surfaces qui ne 
sont pas utilisées pour la récolte et la défense des cultures est alors limité au strict nécessaire. On peut laisser croître les dicotylédones arbustives jusqu’à 2 à 3 mètres de hauteur et cette biomasse est coupée et retourne au sol périodiquement. Les bénéfices potentiels de cette pratique sont d'enrichir la diversité floristique pour favoriser le contrôle biologique des insectes défoliateurs, d'augmenter la production de matière sèche et de recycler des éléments minéraux nutritifs d'horizons plus profonds que ceux exploités par les racines du palmier. Lorsqu'une saison sèche peut faire craindre une compétition pour les réserves hydriques, un rabattage des recrûs avant cette période permet de diminuer ce risque.

\section{Élaborer un outil de fertilisation précis et respectueux de l'environnement}

Le défi de la fertilisation d'une plantation consiste à recommander annuellement, pour chaque unité de fertilisation, les doses de nutriments nécessaires pour satisfaire les besoins des palmiers sans limiter le rendement. Les doses préconisées doivent aussi être ajustées au plus près pour des raisons économiques et environnementales (bilan carbone des pratiques, risques liés aux excès de $\mathrm{N}, \mathrm{K}$, etc.). Il y a donc une double nécessité d'accroître la précision du diagnostic et des recommandations : d'une part, avec la définition des teneurs de référence à atteindre pour chaque sol et chaque matériel végétal et, d'autre part, dans les recommandations pour atteindre ces objectifs sur toutes les surfaces plantées.

\section{Précision des recommandations et des tables de fertilisation}

D'une manière générale, la précision des recommandations et des tables de fertilisation est très dépendante de la qualité des résultats d'analyse des sols et des tissus végétaux. Il faut donc s'appuyer sur un excellent laboratoire, qui garantisse à long terme la stabilité et la répétabilité des résultats. Ce laboratoire doit être accrédité pour l'analyse des sols et des végétaux et participer régulièrement à des enquêtes inter-laboratoires nationales ou internationales. Il doit pouvoir justifier en toute transparence de son bon fonctionnement en partageant des valeurs statistiques sur la précision des analyses (valeurs moyennes et écart-types obtenus pour des échantillons de référence).

Cette précaution est indispensable pour fournir des données fiables et construire un outil d'aide à la décision basé sur des analyses foliaires et des analyses de sol. Une teneur foliaire de référence est un indicateur synthétique simple et bon marché qui garantit un état physiologique correct ne limitant pas le potentiel de rendement. Cet indicateur est calibré au moyen d'essais rigoureux et précis, pour chaque élément, en fonction du climat et du matériel végétal propre à chaque plantation.

Le diagnostic foliaire fonctionne sur des périodes d'environ trois années successives pour corriger les teneurs qui dévient par rapport aux valeurs recommandées. Mais les réseaux d'essais agronomiques montrent toutefois qu'il existe aussi des variations interannuelles des teneurs qui ne s'expliquent pas par les traitements appliqués. 
Elles sont probablement dues à des variations temporelles des flux d'éléments absorbés ou à des rééquilibrages entre organes. Ces variations seraient déterminées par le fonctionnement physiologique du palmier et les facteurs qui agissent sur la production de biomasse. Il peut s'agir d'une variation discontinue de la biomasse foliaire (épanouissement de plusieurs feuilles consécutives pour les climats montrant une saison sèche), de la biomasse des racines ou de cycles de production de régimes. Les variations interannuelles de rendements sont connues et elles induisent probablement des flux d'éléments qui se répercutent sur les teneurs foliaires. Il faut donc interpréter les brusques variations que l'on observe certaines années avec beaucoup de prudence. À l'avenir, le diagnostic foliaire devra évoluer vers un outil qui tienne compte de ces variations interannuelles. Il devra intégrer les variables que l'on utilise pour les modèles de prédiction de rendement et l'absorption des éléments. Ce sont principalement les variables qui agissent sur l'activité photosynthétique (données climatiques et réserves en eau des sols). L'évolution vers un outil générique à plusieurs variables devra s'appuyer sur des bases de données robustes (données météorologiques, productivité mensuelle par unité de fertilisation, rapports d'épandage d'engrais, propriétés des sols des unités de fertilisation) pour tester des hypothèses qui permettent d'accéder à la compréhension de la nutrition minérale en tant que facteur de rendement.

\section{Précision spatiale de l'échantillonnage foliaire et de l'apport d'engrais}

Le premier niveau de précision spatiale pour le diagnostic foliaire est le prélèvement des échantillons. C'est pourquoi la constitution des unités de fertilisation et la sélection des palmiers qui alimentent les échantillons foliaires sont deux étapes clefs pour obtenir une recommandation précise pour une partie significative de chaque unité de fertilisation. Des échantillons spéciaux complètent ce dispositif pour détecter les dérives éventuelles sur les faciès minoritaires de chaque unité. On peut donc envisager une précision à une échelle inférieure à celle de l'unité de fertilisation, mais cela demande de maîtriser parfaitement des applications d'engrais spécifiques au sein des parcelles. La limite de la précision spatiale est celle du palmier planté. L'épandage individuel différencié en fonction de la position géographique de chaque palmier est techniquement possible lorsque l'épandage peut être mécanisé et asservi à un relevé de position par un GPS. Cette mécanisation est difficile et parfois non recommandée (protection des sols) lorsque le relief est accidenté : or c'est probablement avec ces hétérogénéités intra-parcellaires marquées, qu'il semble indiqué d'adapter les recommandations (variations des potentiels de rendement et donc de la demande, variation de l'efficience de la fertilisation). On peut cependant espérer pouvoir délimiter au sein des unités de fertilisation des populations de palmiers qui recevront une fertilisation différenciée si l'on dispose d'un outil d'aide à la décision suffisamment précis pour intégrer cette variabilité.

Il n'est pour l'instant pas possible d'accéder à une recommandation précise pour chaque palmier. Des essais ont testé l'analyse d'images satellitaires pour traduire une composition supposée du feuillage via sa signature spectrale, en un besoin en éléments 
nutritifs, mais aucun algorithme efficace n'a été trouvé pour décrypter les valeurs spectrales des pixels qui représentent chaque palmier. À cela s'ajoute les difficultés à disposer régulièrement d'images de bonne qualité en raison de l'abondance des nuages dans les régions de culture du palmier à huile. À ce stade, cette technologie ne semble donc pas, dans l'immédiat, adaptée aux besoins. 



\section{Conclusion : des outils génériques pour optimiser la fertilisation de chaque plantation}

La méthode que nous proposons pour ajuster les recommandations d'engrais pour qu'elles correspondent le plus exactement possible aux besoins des cultures est un ensemble de procédures. Elle implique de faire des choix lors de la création des unités de fertilisation et la sélection des points d'échantillonnage foliaire et de sol pour suivre chaque unité. Parallèlement, il faut acquérir des résultats expérimentaux pour définir le référentiel de teneurs optimales propre à chaque situation. La démarche est évolutive car elle propose d'acquérir de l'information en continu sur le fonctionnement de la plantation et générer des ajustements pour certains secteurs.

Cependant, ce processus n'est pas applicable partout et n'est pas adoptable par tous. Dans l'état il semble réservé à des plantations agro-industrielles d'une certaine taille. À partir d'une centaine d'hectares, on peut envisager de disposer annuellement d'analyses foliaires et on peut mettre en place des tests d'absorption. À partir de quelques milliers d'hectares, l'investissement dans un ou plusieurs essais de fertilisation pilotés par un ingénieur agronome doté de bonnes bases scientifiques peut rapidement s'avérer rentable. Comment fait-on pour les exploitants de taille modeste qui ne peuvent disposer d'autant de données précises pour leur exploitation?

Il existe des perspectives sérieuses de développer des outils génériques qui prendraient en compte les spécificités de chaque site (propriétés des sols, climat et matériel végétal). Ces outils devront être mieux corrélés avec le rendement. L'objectif est d'expliquer les variations des teneurs foliaires que l'on peut observer d'une année sur l'autre mais aussi les variations dans l'espace (entre parcelles ou unités de sol) lorsqu'elles sont dues aux cycles de production de biomasse. Ces outils devront prendre en compte davantage de variables que les analyses foliaires en particulier celles qui conditionnent la demande liée à la production de biomasse et celles qui agissent sur l'efficience de la fertilisation. La mise au point d'outils robustes n'est envisageable qu'en modélisant des jeux de données sur de longues périodes qu'il s’agisse de résultats expérimentaux ou de suivi de plantations. Leur qualité et en particulier les précautions qui auront été prises pour échantillonner les sols et les palmiers seront primordiales pour que se dégagent des relations explicatives et que l'on puisse définir les jeux d'indicateurs les plus performants pour alimenter les outils.

Nul doute que ces travaux permettront de dépasser l'objectif de précision des recommandations que l'on se fixe au départ; ils permettront de comprendre les déterminants de la productivité et d'en tirer des enseignements sur les pratiques culturales qui 
favorisent les plus hauts rendements en particulier ceux liés aux services écosystémiques rendus par le sol. Ces connaissances intéressent toutes les catégories de producteurs indépendamment de leur taille et constituent donc un formidable bras de levier pour augmenter la productivité des petits exploitants dont les performances sont souvent inférieures aux rendements potentiels exprimés par les agro-industries. Or ces exploitants couvrent environ $40 \%$ des surfaces cultivées dans le monde et leur offrir une perspective de développement durable répond aux exigences de notre époque. Il s'agit d'atteindre notre objectif : satisfaire le besoin mondial en corps gras d'origine végétale tout en limitant les risques de déforestation ainsi que les effets indésirables sur les ressources en eau et les populations locales. 


\section{Bibliographie}

Banabas M., Turner M. A., Scotter D. R. and Nelson P. N., 2008. Losses of nitrogen fertiliser under oil palm in Papua New Guinea: 1. Water balance, and nitrogen in soil solution and runoff. Australian Journal of Soil Research, 46(4): 332-339. https://doi.org/10.1071/SR07171

Caliman J. P., Olivin J., et Dufour, O., 1987. Dégradation des sols ferrallitiques sableux en culture de palmiers à huile par acidification et compaction. Oléagineux, 42(11) : 394-401. http://agritrop.cirad.fr/453135/

Caliman J. P., Daniel C. et Tailliez B., 1994. La nutrition minérale du Palmier à Huile - Oil palm mineral nutrition. Plantations, Recherche, Développement, 3 : 36-54. http://agritrop.cirad.fr/387369/

Cirad-IRHO, 1969. Les symptômes de carence en magnésium chez le palmier à huile. Pratique Conseils de l'IRHO nº 80. Oléagineux, 24(1) : 11-12.

Cirad-IRHO, 1991. La carence azotée chez le palmier à huile. Symptômes et correction. PratiqueConseils de l'IRHO n 320. Oléagineux, 46(6) : 247-250.

Cirad-IRHO, 1992. La carence potassique chez le palmier à huile. Symptômes et correction. Pratique - Conseils de l'IRHO n 332. Oléagineux, 47(10) : 587-591.

Cirad-IRHO, 1992. La déficience en bore chez le palmier à huile : symptômes et corrections. Pratique - Conseils de l'IRHO n 334. Oléagineux. 47(12) : 719-725.

Cirad-IRHO, 1977. Préparation et conditionnement des échantillons pour le diagnostic foliaire du palmier à huile et du cocotier. Pratique - Conseils de l'IRHO n 170. Oléagineux, 32(3) : 95-99.

Cirad-IRHO, 1977. Le diagnostic foliaire pour le contrôle de la nutrition des plantations de palmier. Prélèvement des échantillons foliaires. Pratique - Conseils de l'IRHO n 172 . Oléagineux, 32(5) : 211-216.

Dassou O., Nodichao L., Ollivier J., Impens R., Dubos B., Bonneau X., De Raïssac M., Durand-Gasselin T., Sinsin B., Van Damme P., 2018. Oil Palm (Elaeis guineensis Jacq.) Leaf K and $\mathrm{Mg}$ Contents Differ with Progenies: Implications and Research Needs. In Tielkes E. (ed.), Tropentag 2018, Global food security and food safety: the role of Universities, Gand, Belgique, 17-19 September 2018. Weikersheim, Margraf Publishers GmbH, p. 230. ISBN 978-3-8236-1760. http://agritrop.cirad.fr/590883/ ; http://www.tropentag.de/.

Dubos B., Caliman J.-P., Corrado F., Quencez P., Siswo S. et Tailliez B., 1999. Rôle de la nutrition en magnésium chez le palmier à huile. Plantations, Recherche, Développement, 6(5) : 313-325. http://agritrop.cirad.fr/264349/

Dubos B., Alarcón W. H., López J. E. and Ollivier J., 2011. Potassium uptake and storage in oil palm organs: the role of chlorine and the influence of soil characteristics in the Magdalena valley, Colombia. Nutrient Cycling in Agroecosystems, 89(2): 219-227. https://doi.org/10.1007/s10705-010-9389-x

Dubos B., Baron V., Bonneau X., Dassou O., Flori A., Impens R., Ollivier J., Pardon L., 2019. Precision agriculture in oil palm plantations: diagnostic tools for sustainable $\mathrm{N}$ and Knutrient supply. OCL, 26(5). https://doi.org/10.1051/ocl/2019001 
Dubos, B., Baron, V., Bonneau, X., Flori, A., \& Ollivier, J. (2017a). High soil calcium saturation limits use of leaf potassium diagnosis when $\mathrm{KCl}$ is applied in oil palm plantations. Experimental Agriculture, 54(5) : 1-11. https://doi.org/10.1017/S0014479717000473

Dubos B., Snoeck D. and Flori A., 2017b. Excessive use of fertilizer can increase leaching processes and modify soil reserves in two Ecuadorian oil palm plantations. Experimental Agriculture, 53(02): 255-268. https://doi.org/10.1017/S0014479716000363

Dubos B. and Flori A., 2014. Persistence of Mineral Fertility Carried over from the First Crop Cycle in Two Oil Palm Plantations in South America. Oil Palm Bulletin, 68: 8-15. http://palmoilis.mpob.gov.my/index.php/opb

Fairhurst T. and Caliman J.-P., 2001. Symptômes de Déficiences Minérales et Anomalies chez le Palmier à Huile (Elaeis guineensis Jacq.) : Description, Origine, Prévention, Correction. Guide de poche. Singapour, PPI-CIRAD-CP, Série Palmier à huile : ESEAP 7, 60 p. ISBN 981-04-4840-6. http://agritrop.cirad.fr/510748/

Foster H. L., 2003. Assessment of Oil Palm Fertilizer Requirements. In Fairhurst T. and Hardter R. (eds.), Oil Palm: Management for Large and Sustainable Yields. Potash \& Phosphate Institute (PPI), Potash \& Phosphate Institute of Canada (PPIC), and International Potash Institute (IPI, Basel). pp. 191-230.

Foster H. L. and Prabowo N. E., 1996. Variation in potassium fertiliser requirements in oil palm in North Sumatra. Presented at the 1996 PORIM International Palm Oil Congress : Competitiveness for the 21st Century, Kuala Lumpur Malaysia: PORIM.

Gerendás J., Donough C. and Oberthur T., 2009. Sulphur Nutrition of Oil Palm in Indonesia The Neglected Macronutrient. Proceedings of Agriculture, Biotechnology \& Sustainability Conference, 2: 27-31.

Goh K. J., Hardter R., and Fairhurst T., 2003. Fertilizing for maximum return In Fairhurst T. and Hardter R. (eds.), Oil Palm: Management for Large and Sustainable Yields. Potash \& Phosphate Institute (PPI), Potash \& Phosphate Institute of Canada (PPIC), and International Potash Institute (IPI, Basel). pp. 279-306.

Jacquemard J., Ollivier J., Erwanda Edyana Suryana, and Pepep Permadi, 2009. Genetic signature in mineral nutrition in oil palm (Elaeis guineensis Jacq.): a new panorama for high yielding materials at low fertiliser cost. In PIPOC 2009 International Palm Oil Congress, vol. 2, Malaysia. pp. 337-373.

Nelson P. N., Banabas M., Goodrick I., Webb M. J., Huth N. I. and O’Grady D., 2015. Soil sampling in oil palm plantations: a practical design that accounts for lateral variability at the tree scale. Plant and Soil, 394(1-2): 421-429. https://doi.org/10.1007/s11104-015-2490-9

$\mathrm{Ng}$ S. K. and Thamboo S., 1967. Nutrient contents in Oil Palm in Malaya 1. Nutrients required for reproduction: fruit bunches and male inflorescences. The Malaysian Agricultural Journal, 46(1) :3-45.

Ollagnier M. and Ochs R., 1971. Chlorine, a new essential element il oil palm nutrition. Oléagineux, 26(1) : 1-15.

Ollagnier M. and Ochs R., 1972. Sulphur deficiencies in the oil palm and coconut. Oléagineux, 27(4): 193-198. Retrieved from Oléa.

Ollagnier M. and Ochs R., 1981. Management of mineral nutrition on Industrial Oil Palm Plantation - Fertilizer Savings. Oléagineux, 36(8-9) : 409-421.

Rincón Numpaque A. H. and Torres Aguas J. S., 2015. Extracción de nutrimentos en racimos de palma híbrida Elaeis oleifera $x$ Elaeis guineensis $(\mathrm{O} \times \mathrm{G})$ en la Zona Suroccidental. Estudio preliminar. Ceniavances, (182) : 1-8. 
Tampubolon F. C., Daniel C. and Ochs R., 1989. Oil palm responses to nitrogen and phosphate fertilizer in Sumatra. Presented at the 1989 PORIM International Palm Oil Development Conference, Kuala Lumpur. pp. 419-428.

Tan G. Y. and Rajaratnam J. A., 1978. Genetic variability of leaf nutrient concentration in oil palm. Crop Science, 18 (July August): 548-550.

Tarmizi A. M. and Mohd Tayeb D., 2006. Nutrient Demands of Tenera Oil Palm Planted on Inland Soils of Malaysia. Journal of Oil Palm Research, 18(1) : 204-209.

Teoh, K. C. and Chew P. S., 1987. Potassium in the oil palm ecosystem and some implications to manuring practice). Presented at the 1987 International Oil Palm / Palm Oil Conferences : Progress and Prospects, Kuala Lumpur, Malaysia. pp. 277-286.

Webb, M. J., 2009. A conceptual framework for determining economically optimal fertiliser use in oil palm plantations with factorial fertiliser trials. Nutrient Cycling in Agroecosystems, 83(2) : 163-178. https://doi.org/10.1007/s10705-008-9207-x

Webb M. J., Berthelsen S., Nelson P., van Rees H., 2009. Final Report. Overcoming magnesium deficiency in oil palm crops on volcanic ash soils of Papua New Guinea. Canberra, ACIAR, Report FR2009-11. ISBN 97819215316 4. https://www.aciar.gov.au/node/11486

Yates M., 1964. The Design and analysis of factorial experiment. Commonwealth Agricultural Bureaux, Bucks, 98 p. Public Library of India https://archive.org/details/in.ernet.dli.2015.449111/ page/n1 
Mise en page : Hélène Bonnet - Studio 9 Impression : ISIPRINT

Dépôt légal : octobre 2020 
Guide d'aide à la conception de la fertilisation des plantations de palmier à huile, cet ouvrage expose dans un langage clair comment définir dans chaque plantation les recommandations d'engrais qui prennent en compte les spécificités de chaque site. Les auteurs présentent les principes d'interprétation des résultats d'analyse des tissus végétaux, principalement les folioles, en tenant compte de l'influence des facteurs propres à chaque plantation (âge, matériel végétal, climat, sol). Ils détaillent comment on détermine expérimentalement les teneurs de référence en éléments minéraux spécifiques de la plantation et les règles d'échantillonnage pour suivre l'état nutritionnel de la plantation. La priorité est donnée aux faciès (sol, matériel végétal) les plus spécifiques des surfaces plantées plutôt qu'à une représentation moyenne des parcelles. Ce guide explique comment créer des barèmes de fertilisation pour atteindre les teneurs foliaires optimales tirées de l'expérimentation, et confronter ces données à des informations issues des systèmes d'information géographiques. Ce système d'aide à la décision est conçu pour être valable dans toutes les situations. Au-delà de l'optimisation économique de la fertilisation, les préoccupations environnementales sont prises en compte : la santé des sols et l'efficience de l'absorption doivent être intégrées dans la stratégie de fertilisation en optimisant les pratiques culturales pour l'application des engrais et la gestion de la matière organique. Ce guide est illustré par de nombreux exemples issus des essais dans des conditions variées de sol, de climat et de matériel végétal en Afrique et en Amérique latine.

Bernard Dubos, chercheur au Cirad, a étudié les outils de diagnostic des besoins en nutriments des plantations de palmier à huile tout au long de sa carrière. En partenariat avec des sociétés privées en Afrique et en Amérique latine, il a construit un réseau d'essais pour optimiser les stratégies de fertilisation de chaque plantation et préserver leur durabilité.

Xavier Bonneau, agronome spécialiste du cocotier et du palmier à huile au Cirad, a mis en place un conseil agronomique pour améliorer les rendements et la durabilité de plantations, notamment en Afrique et en Indonésie. Les impacts environnementaux des pratiques agricoles sont pris en compte dans ses travaux.

\begin{abstract}
Albert Flori, agronome et statisticien au Cirad, apporte ses compétences à l'amélioration des systèmes de culture pérennes tropicaux en élaborant des protocoles expérimentaux et des aides à l'interprétation fiabilisant les décisions concernant la fertilisation des plantations et le choix du matériel végétal.
\end{abstract}

En couverture : éficience en potassium sur palmier à huile (hybride interspécifique) en Equateur / Régimes de l'hybride Coari sur gare de récolte / Mucuna bracteata en plantation de palmier à huile. (c) Bernard Dubos
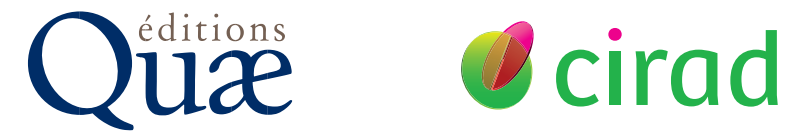

Éditions Cirad, Ifremer, INRAE www.quae.com

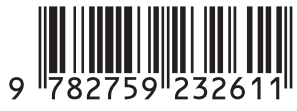

ISSN : 1952-1251

Réf. : 02766 